\title{
THE YAMABE PROBLEM
}

\author{
JOHN M. LEE AND THOMAS H. PARKER
}

\section{Contents}

\section{Introduction}

2. Geometric and analytic preliminaries

3. The model case: the sphere

4. The variational approach

5. Conformal normal coordinates

6. Stereographic projections

7. The test function estimate

8. General relativity

9. Analysis on asymptotically flat manifolds

10. The positive mass theorem

11. Solution of the Yamabe problem

Appendix: Witten's proof

Bibliography

1. Introduction. Riemannian differential geometry originated in attempts to generalize the highly successful theory of compact surfaces. From the earliest days, conformal changes of metric (multiplication of the metric by a positive function) have played an important role in surface theory. For example, one consequence of the famous uniformization theorem of complex analysis is the fact that every surface has a conformal metric of constant (Gaussian) curvature. This provides a "standard model" for each homeomorphism class of surfaces, and reduces topological questions to differential geometric ones.

Life would be simple if the naive generalization of this theorem held in higher dimensions: every $n$-manifold would have a conformal metric of constant curvature, and questions in differential topology would be reduced to geometric questions about the constant-curvature models. However, it is easy to see that this cannot be true. In general the problem is highly overdetermined: the curvature tensor has on the order of $n^{4}$ independent components, while a conformal change of metric allows us to choose only one unknown function. For example, if $n \geqslant 4$, the Weyl tensor, formed from the components of the Riemannian curvature tensor, is conformally invariant and vanishes if and only if the metric is locally conformally equivalent to the Euclidean metric. From this point of view it seems natural instead to seek a

Received by the editors June 24, 1986.

1980 Mathematics Subject Classification. Primary 53A30; Secondary 35J20, 35J60.

Research of the first author supported in part by a research grant from the National Science Foundation. 
conformal change of metric that makes only the scalar curvature (the complete contraction of the curvature tensor) constant, for then we are looking for one unknown function to satisfy one condition. Thus we are led to:

The Yamabe Problem. Given a compact Riemannian manifold $(M, g)$ of dimension $n \geqslant 3$, find a metric conformal to $g$ with constant scalar curvature.

In 1960, H. Yamabe [Y] attempted to solve this problem using techniques of calculus of variations and elliptic partial differential equations. He claimed that every compact Riemannian $n$-manifold $M$ has a conformal metric of constant scalar curvature. Unfortunately, his proof contained an error, discovered in 1968 by Neil Trudinger [T]. Trudinger was able to repair the proof, but only with a rather restrictive assumption on the manifold $M$. In order to understand the restriction, let us describe Yamabe's approach.

Suppose $(M, g)$ is a compact Riemannian manifold of dimension $n \geqslant 3$ (which we will always assume is connected). Any metric conformal to $g$ can be written $\tilde{g}=e^{2 f} g$, where $f$ is a smooth real-valued function on $M$. If $S$ and $\tilde{S}$ denote the scalar curvatures of $g$ and $\tilde{g}$, respectively, they satisfy the transformation law:

$$
\tilde{S}=e^{-2 f}\left(S+2(n-1) \Delta f-(n-1)(n-2)|\nabla f|^{2}\right),
$$

in which $\Delta f$ denotes the Laplacian of $f$ and $\nabla f$ its covariant derivative, defined with respect to the metric $g$. This formula is considerably simplified if we make the substitution $e^{2 f}=\varphi^{p-2}$, with $p=2 n /(n-2)$ and $\tilde{g}=\varphi^{p-2} g$ :

$$
\tilde{S}=\varphi^{1-p}\left(4 \frac{n-1}{n-2} \Delta \varphi+S \varphi\right) \text {. }
$$

Notation. Throughout this paper, we will use the following notations:

$$
n=\operatorname{dim} M \geqslant 3 ; \quad p=\frac{2 n}{n-2}, \quad a=4 \frac{n-1}{n-2} ; \quad \square=a \Delta+S .
$$

Thus $\tilde{g}=\varphi^{p-2} g$ has constant scalar curvature $\lambda$ iff $\varphi$ satisfies the Yamabe equation:

$$
\square \varphi=\lambda \varphi^{p-1} .
$$

This is a sort of "nonlinear eigenvalue problem." The analytic properties of the equation $\square \varphi=\lambda \varphi^{q}$ depend critically on the value of the exponent $q$ : when $q=1$, the equation is just the linear eigenvalue problem for $\square$. When $q$ is close to 1 , as we will see in $\S 4$, its analytic behavior is quite similar to that of the linear case, and the problem is easily solved. When $q$ is very large, the methods based on linear theory break down altogether. It happens that the exponent $q=p-1=(n+2) /(n-2)$ that occurs in the Yamabe equation is precisely the critical value, below which the equation is easy to solve and above which it may be impossible. This accounts for the analytic complexity of the Yamabe problem.

Yamabe observed that equation (1.2) is the Euler-Lagrange equation for the functional

$$
Q(\tilde{g})=\frac{\int_{M} \tilde{S} d V_{\tilde{g}}}{\left(\int_{M} d V_{\tilde{g}}\right)^{2 / p}},
$$


where $\tilde{g}$ is allowed to vary over metrics conformally equivalent to $g$. To see this, observe that $Q$ can be written $Q(\tilde{g})=Q\left(\varphi^{p-2} g\right)=Q_{g}(\varphi)$, where

$$
\begin{gathered}
Q_{g}(\varphi)=E(\varphi) /\|\varphi\|_{p}^{2}, \\
E(\varphi)=\int_{M} a|\nabla \varphi|^{2}+S \varphi^{2} d V_{g}, \quad\|\varphi\|_{p}=\left(\int_{M}|\varphi|^{p} d V_{g}\right)^{1 / p} .
\end{gathered}
$$

Then for any $\psi \in C^{\infty}(M)$, integration by parts yields

$$
\left.\frac{d}{d t} Q_{g}(\varphi+t \psi)\right|_{t=0}=\frac{2}{\|\varphi\|_{p}^{2}} \int_{M}\left(a \Delta \varphi+S \varphi-\|\varphi\|_{p}^{-p} E(\varphi) \varphi^{p-1}\right) \psi d V_{g} .
$$

Thus $\varphi$ is a critical point of $Q_{g}$ if and only if it satisfies the Yamabe equation (1.2) with $\lambda=E(\varphi) /\|\varphi\|_{p}^{p}$.

Since by Hölder's inequality $\left|\int_{M} S \varphi^{2}\right|$ is bounded by a multiple of $\|\varphi\|_{p}^{2}$, it follows easily that $Q_{g}$ (and thus $Q$ ) is bounded below. We set

$$
\begin{aligned}
\lambda(M) & =\inf \{Q(\tilde{g}): \tilde{g} \text { conformal to } g\} \\
& =\inf \left\{Q_{g}(\varphi): \varphi \text { a smooth, positive function on } M\right\} .
\end{aligned}
$$

This constant $\lambda(M)$ is an invariant of the conformal class of $(M, g)$, called the Yamabe invariant. Its value is central to the analysis of the Yamabe problem.

The solution of the Yamabe problem follows its historical development. It is summarized by three main theorems.

Trudinger's modification of Yamabe's proof worked whenever $\lambda(M) \leqslant 0$. In fact, he showed that there is a positive constant $\alpha(M)$ such that the proof works when $\lambda(M)<\alpha(M)$. Now it is easy to show (see §3) that $\lambda(M) \leqslant$ $\lambda\left(S^{n}\right)$, where $S^{n}$ is the sphere with its standard metric. In 1976, Thierry Aubin [A2] extended Trudinger's result by showing, in effect, that $\alpha(M)=\lambda\left(S^{n}\right)$ for every $M$. This established:

Theorem A (Yamabe, Trudinger, Aubin). The Yamabe problem can be solved on any compact manifold $M$ with $\lambda(M)<\lambda\left(S^{n}\right)$, where $S^{n}$ is the sphere with its standard metric.

This result shifts the focus of the proof from analysis to the problem of understanding the essentially geometric invariant $\lambda(M)$. The obvious approach to showing that $\lambda(M)<\lambda\left(S^{n}\right)$ is to find a "test function" $\varphi$ with $Q_{g}(\varphi)<\lambda\left(S^{n}\right)$. Aubin [A2] sought such a function compactly supported in a small neighborhood of a point $P \in M$. By carefully studying the local geometry of $M$ near $P$ in normal coordinates, he was able to construct such test functions in many cases, proving the following theorem.

TheOREM B (AuBIN). If $M$ has dimension $n \geqslant 6$ and is not locally conformally flat then $\lambda(M)<\lambda\left(S^{n}\right)$.

The remaining cases are more difficult because the local conformal geometry does not contain sufficient information to conclude that $\lambda(M)<\lambda\left(S^{n}\right)$. These cases thus require the construction of a global test function. This was done by Richard Schoen [S] in 1984. His theorem completes the solution of the Yamabe problem. 
Theorem C (Schoen). If $M$ has dimension 3, 4, or 5, or if $M$ is locally conformally flat, then $\lambda(M)<\lambda\left(S^{n}\right)$ unless $M$ is conformal to the standard sphere.

Schoen's proof introduced two important new ideas. First, he recognized the key role of the Green function for the operator $\square$; in fact, his test function was simply the Green function with its singularity smoothed out. Second, he discovered the unexpected relevance of the positive mass theorem of general relativity, which had recently been proved in dimensions 3 and 4 by Schoen and S.-T. Tau [SY1, SY2, SY4]. A curious feature of Schoen's proof is that it works only in the cases not covered by Aubin's theorem.

The proof of Theorem $\mathrm{C}$ actually requires an $n$-dimensional version (as yet unpublished) of the positive mass theorem, which was announced by Schoen in [S]. The 5-dimensional case appears to be a straightforward generalization of the 4-dimensional proof in [SY2]. The higher-dimensional case is more difficult. However, for $n \geqslant 6$ the result is needed only for locally conformally flat manifolds; Schoen and Yau [SY6] have recently given an alternate proof for this case (see $§ 10)$.

The solution of the Yamabe problem marks a milestone in the development of the theory of nonlinear partial differential equations. Semilinear equations of the form (1.2) with critical exponent arise in many contexts and have long been studied by analysts. This is the first time that such an equation has been completely solved.

The aim of the present paper is to provide a unified expository account of the proof of the Yamabe theorem, presenting for the first time the complete solution in one place. This account should be accessible to anyone familiar with enough differential geometry to feel comfortable with tensors, covariant derivatives, and normal coordinates; and enough analysis to follow arguments involving Sobolev and Hölder spaces and basic elliptic regularity theory for the Laplace operator.

The proof we present is self-contained (except for a central step in the positive mass theorem), and incorporates several improvements over the proofs currently available in the literature. Most importantly, we show how to recast the local proof of Aubin and the global proof of Schoen in a single framework.

The key simplification is achieved by introducing a special coordinate system, called "conformal normal coordinates". These are analogous to geodesic normal coordinates on a Riemannian manifold, and greatly simplify local analysis on conformal manifolds. (A related coordinate system was invented by Robin Graham [G] to study conformal invariant theory.)

Using the Green function for $\square$, we define a "stereographic projection" from $M$ minus a point to a noncompact manifold $\hat{M}$ with zero scalar curvature. We then construct a test function on $\hat{M}$ whose Yamabe quotient is very close to that of the sphere. Conformal normal coordinates allow us to obtain a precise estimate of this Yamabe quotient. This shows that $\lambda(M)<\lambda\left(S^{n}\right)$ provided a certain quantity called the "distortion coefficient" is positive. This coefficient measures the average behavior of the metric on $\hat{M}$ near infinity. In the case of Theorem $\mathrm{B}$, it is readily computed from the local conformal geometry of $M$, 
while in the case of Theorem $\mathrm{C}$ its positivity follows from the positive mass theorem.

This approach, we feel, sheds considerable light on the relationship between Theorems B and C, and eliminates the need for the "delicate perturbation argument" that Schoen used to handle dimensions 4 and 5. It also shows that the expansion of the Green function contains all the information needed to prove that $\lambda(M)<\lambda\left(S^{n}\right)$.

In $\S 2$ of this paper, we recall the notation and background material from differential geometry and analysis that we will be using throughout the paper. $\$ 3$ is a discussion of the "model case" for the Yamabe problem, the sphere with its standard metric. In $\S 4$, we complete the analytic part of the proof by showing that the problem can be solved if $\lambda(M)<\lambda\left(S^{n}\right)$.

Conformal normal coordinates are introduced in $\$ 5$, and used to give a very simple proof of Aubin's theorem. In $\S 6$, we define a stereographic projection for a compact manifold with positive Yamabe invariant, and derive the asymptotic expansion of its metric explicitly to high order. In $\S 7$ we construct a test function on $\hat{M}$ and compute its Yamabe quotient.

$\S \S 8,9$, and 10 give a brief treatment of the positive mass theorem. In $\S 8$, we introduce the physicists' notion of mass of an asymptotically flat manifold, and describe the physical motivation for the positive mass and positive action conjectures. $\$ 9$ discusses some analytic tools for asymptotically flat manifolds, and $\$ 10$ sketches a proof of the $n$-dimensional positive mass theorem. Finally, in $\$ 11$ we complete the proof of the Yamabe theorem.

We are indebted to Karen Uhlenbeck, who first introduced us to the Yamabe problem, and to David Jerison, whose ideas about the expansion of the Green function were very helpful in early conversations. We would also like to thank Jean-Pierre Bourguignon for helpful comments on the manuscript.

2. Geometric and analytic preliminaries. In this section we collect some notations and well-known facts from differential geometry and the theory of linear partial differential equations on manifolds. Most of the results stated here without proof can be found in [KN, A3, or GT].

Geometric notations. We use standard index notation for tensors. If $g_{j k}$ are the components of the metric tensor with respect to a coordinate system $\left\{x^{i}\right\}$, $g_{j k}$ and its inverse $g^{j k}$ are used to raise and lower indices: $T_{k}^{l}=g^{j l} T_{j k}$. The metric extends to an inner product on tensors of any type: for example, the norm of the 2-tensor $T$ with components $T_{j k}$ is $|T|^{2}=T_{j k} T^{j k}=g^{j l} g^{k m} T_{j k} T_{l m}$.

Covariant differentiation is denoted by $\nabla$. If $f$ is a function on $M$, its covariant derivative is the 1 -tensor $\nabla f$ whose components we will write $f_{j}$. The $m$ th covariant derivative of $f$ is the $m$-tensor $\nabla^{m} f$, with components $f_{i_{1} i_{2} \cdots i_{m}}$. If $T$ is a tensor, indices of $\nabla^{m} T$ that result from differentiation will be separated by a comma: for example, if $T_{j k}$ are the components of a 2-tensor $T$ as above, then the components of $\nabla^{2} T$ are denoted by $T_{j k, l m}$.

The Euclidean volume form $d x^{1} \wedge \cdots \wedge d x^{n}$ on $\mathbf{R}^{n}$ will be denoted by $d x$. The standard volume form on the unit $(n-1)$-sphere in $\mathbf{R}^{n}$ will be denoted by $d \omega$, and we write $d \omega_{r}=r^{n-1} d \omega$ for the volume form on the sphere $S_{r}$ of radius $r$. We let $\omega$ denote the volume of the unit sphere, and $\omega_{r}=r^{n-1} \omega$. If $g$ 
is a Riemannian metric, $d V_{g}$ denotes its Riemannian density, which is defined whether or not $M$ is oriented. In local coordinates, $d V_{g}=(\operatorname{det} g)^{1 / 2}|d x|$.

The divergence operator is the formal adjoint $\nabla^{*}$ of $\nabla$, given on 1-forms by $\nabla^{*} \omega=-\omega_{i}{ }^{i}$. On a compact manifold with boundary it satisfies the divergence theorem

$$
\int_{M} \nabla^{*} \omega d V_{g}=-\int_{\partial M} \omega(N) d V_{\tilde{g}}
$$

where $\tilde{g}$ is the induced metric on $\partial M$ and $N$ is the outward unit normal. (If $M$ is oriented this is just Stokes' theorem. If not, it follows from Stokes' theorem on the oriented double cover of $M$.) The Laplacian is the second-order differential operator $\Delta$ given on functions by

$$
\Delta u=\nabla^{*} \nabla u=-(\operatorname{det} g)^{-1 / 2} \partial_{i}\left(g^{i j}(\operatorname{det} g)^{1 / 2} \partial_{j} u\right) .
$$

On a compact manifold without boundary the divergence theorem yields the "integration by parts" formula

$$
\int_{M}\langle\nabla v, \nabla u\rangle d V_{g}=\int_{M} v \Delta u d V_{g}
$$

The Riemannian curvature tensor is the tensor with components $R_{i j k l}$, computed in a coordinate system $\left\{x^{i}\right\}$ by:

$$
R_{i j k l}=\left\langle R\left(\partial_{k}, \partial_{l}\right) \partial_{j}, \partial_{i}\right\rangle \text {, }
$$

where $R$ is the curvature operator $R(V, W)=\left[\nabla_{V}, \nabla_{W}\right]-\nabla_{[V, W]}$. It satisfies the Ricci identity

$$
\omega_{j, k l}-\omega_{j, l k}=R_{j k l}^{i} \omega_{i}
$$

for any one-form $\omega_{j} d x^{j}$, and the Bianchi identities:

$$
R_{i j k l}+R_{i k l j}+R_{i l j k}=0, \quad R_{i j k l, m}+R_{i j l m, k}+R_{i j m k, l}=0 .
$$

The Ricci tensor is the contraction $R_{j l}=R_{j k l}^{k}$ of the curvature tensor, and the scalar curvature is the trace $S=R_{j}{ }^{j}$ of the Ricci tensor. $M$ is said to be Einstein if its Ricci tensor is a scalar multiple of the metric, or equivalently if the traceless Ricci tensor $B_{i j}=R_{i j}-(S / n) g_{i j}$ vanishes. Contracting the second equation in (2.3) on the indices $i, k$ and again on $j, l$, we obtain:

$$
S_{, m}-2 R_{m, i}^{i}=0 \text {. }
$$

It follows that an Einstein manifold has constant scalar curvature.

The remaining components of the curvature tensor constitute the Weyl tensor $W$, with components:

$$
\begin{aligned}
W_{i j k l}= & R_{i j k l}-\frac{1}{n-2}\left(R_{i k} g_{j l}-R_{i l} g_{j k}+R_{j l} g_{i k}-R_{j k} g_{i l}\right) \\
& +\frac{S}{(n-1)(n-2)}\left(g_{i k} g_{j l}-g_{i l} g_{j k}\right)
\end{aligned}
$$


(This formula is chosen so that the trace of $W$ on any pair of indices vanishes. $W$ vanishes identically if $n=3$.) Using the definitions of $W$ and $B$, we can write the curvature tensor as a sum of three parts:

$$
\begin{aligned}
R_{i j k l}= & W_{i j k l}+\frac{1}{n-2}\left(B_{i k} g_{j l}-B_{i l} g_{j k}+B_{j l} g_{i k}-B_{j k} g_{i l}\right) \\
& +\frac{S}{n(n-1)}\left(g_{i k} g_{j l}-g_{i l} g_{j k}\right) .
\end{aligned}
$$

In particular, if $W=0$ and $B=0$, the curvature tensor is completely determined by the (constant) scalar curvature $S$, and $M$ is said to have constant curvature. It is well known that a complete, simply connected manifold of constant curvature is isometric to $\mathbf{R}^{n}, S^{n}$, or $n$-dimensional hyperbolic space.

If $\tilde{g}=e^{2 f} g$ is a metric conformal to $g$, one can compute the components of the curvature tensor $\tilde{R}$ of $\tilde{g}$ in terms of those of the curvature of $g$. The results we will need are the transformation laws for the Ricci and scalar curvatures:

$$
\begin{gathered}
\tilde{R}_{j k}=R_{j k}-(n-2) f_{j k}+(n-2) f_{j} f_{k}+\left(\Delta f-(n-2) f_{i} f^{i}\right) g_{j k} \\
\tilde{S}=e^{-2 f}\left(S+2(n-1) \Delta f-(n-1)(n-2) f_{i} f^{i}\right) .
\end{gathered}
$$

As in the introduction, this can be rewritten in the form (1.1). One computes also that the Weyl tensor is conformally invariant: $\tilde{W}_{j k l}^{i}=W_{j k l}^{i}$.

A Riemannian manifold $M$ is said to be locally conformally flat if it is locally conformal to Euclidean space. It is a classical result (see for example [E, §28]) that for $n \geqslant 4$ the Weyl tensor vanishes identically if and only if $M$ is locally conformally flat.

The operator $\square=a \Delta+S$, where $a=4(n-1) /(n-2)$, is called the conformal Laplacian. It is conformally invariant in the following sense. If $\tilde{g}=$ $\varphi^{p-2} g$ (with $p=2 n /(n-2)$ ) is a metric conformal to $g$, and $\tilde{\square}$ is similarly defined with respect to $\tilde{g}$, then computing $\tilde{\Delta}$ in terms of $\Delta$ and using the transformation law (1.1) for scalar curvature, one finds that

$$
\tilde{\square}\left(\varphi^{-1} u\right)=\varphi^{1-p} \square u .
$$

Analytic preliminaries. Suppose $P$ is a linear partial differential operator on a manifold $M$. If $u$ and $f$ are locally integrable functions on $M$, we say $u$ is a weak (or distribution) solution to the equation $P u=f$ if, for every smooth compactly supported function $\varphi$,

$$
\int_{M} u P^{*} \varphi d V_{g}=\int_{M} f \varphi d V_{g}
$$

in which $P^{*}$ is the formal adjoint of $P$, obtained from $P$ by formally integrating by parts. (In particular, $\Delta^{*}=\Delta$.)

There are many function spaces used in solving differential equations; a nonlinear problem such as the Yamabe problem requires the use of a number of them. Therefore, we begin by defining the spaces we will be using. 
If $q \geqslant 1$, the Lebesgue space $L^{q}(M)$ is the set of locally integrable functions $u$ on $M$ for which the norm

$$
\|u\|_{q}=\left(\int_{M}|u|^{q} d V_{g}\right)^{1 / q}
$$

is finite.

If in addition $k$ is a nonnegative integer, the Sobolev space $L_{k}^{q}(M)$ is the set of $u \in L^{q}(M)$ such that $P u=f \in L^{q}(M)$ (in the weak sense) whenever $P$ is a smooth differential operator of order $\leqslant k$. We define the Sobolev norm \|\|$_{q, k}$ on $L_{k}^{q}(M)$ by:

$$
\|u\|_{q, k}=\left(\sum_{i=0}^{k} \int_{M}\left|\nabla^{i} u\right|^{q} d V_{g}\right)^{1 / q} .
$$

The space $C^{k}(M)$ is the set of $k$ times continuously differentiable functions on $M$, for which the norm

$$
\|u\|_{C^{k}}=\sum_{i=0}^{k} \sup _{M}\left|\nabla^{i} u\right|
$$

is finite. Then the Hölder space $C^{k, \alpha}(M)$ is defined for $0<\alpha<1$ as the set of $u \in C^{k}(M)$ for which the norm

$$
\|u\|_{C^{k, \alpha}}=\|u\|_{C^{k}}+\sup _{x, y} \frac{\left|\nabla^{k} u(x)-\nabla^{k} u(y)\right|}{|x-y|^{\alpha}}
$$

is finite, where the supremum is over all $x \neq y$ such that $y$ is contained in a normal coordinate neighborhood of $x$, and $\nabla^{k} u(y)$ is taken to mean the tensor at $x$ obtained by parallel transport along the radial geodesic from $x$ to $y$. As usual, $C^{\infty}(M)$ and $C_{c}^{\infty}(M)$ denote the spaces of smooth functions and smooth compactly supported functions on $M$, respectively. We note that if $M$ is complete, $C_{c}^{\infty}(M)$ is dense in $L_{k}^{q}(M)$.

The relations among these spaces are expressed in the following theorems.

TheOREM 2.1 (SOBOLEV EMBEDDING THEOREMS FOR $\mathbf{R}^{n}$ ).

(a) Suppose

$$
\frac{1}{r}=\frac{1}{q}-\frac{k}{n} .
$$

Then $L_{k}^{q}\left(\mathbf{R}^{n}\right)$ is continuously embedded in $L^{r}\left(\mathbf{R}^{n}\right)$. In particular, for $q=2$, $k=1, r=p=2 n /(n-2)$, we have the following Sobolev inequality:

$$
\|\varphi\|_{p}^{2} \leqslant \sigma_{n} \int_{\mathbf{R}^{n}}|\nabla \varphi|^{2} d x, \quad \varphi \in L_{1}^{2}\left(\mathbf{R}^{n}\right) .
$$

We will call the smallest such constant $\sigma_{n}$ the $n$-dimensional Sobolev constant.

(b) Suppose $0<\alpha<1$, and

$$
\frac{1}{q} \leqslant \frac{k-\alpha}{n} .
$$

Then $L_{k}^{q}\left(\mathbf{R}^{n}\right)$ is continuously embedded in $C^{\alpha}\left(\mathbf{R}^{n}\right)$. 
We can transfer these results to a compact manifold $M$ by covering $M$ with small coordinate patches, applying the above theorems in normal coordinates, and summing the results with a partition of unity. The general results are expressed in the following theorem.

THEOREM 2.2 (SOBOLEV EMBEDDING THEOREMS FOR COMPACT MANIFOLDS). Suppose $M$ is a compact Riemannian manifold of dimension $n$ ( possibly with $C^{1}$ boundary).

(a) If

$$
\frac{1}{r} \geqslant \frac{1}{q}-\frac{k}{n}
$$

then $L_{k}^{q}(M)$ is continuously embedded in $L^{r}(M)$.

(b) (RELlich-KONDRAKOV THEOREM) Suppose strict inequality holds in (a). Then the inclusion $L_{k}^{q}(M) \subset L^{r}(M)$ is a compact operator.

(c) Suppose $0<\alpha<1$, and

$$
\frac{1}{q} \leqslant \frac{k-\alpha}{n}
$$

Then $L_{k}^{q}(M)$ is continuously embedded in $C^{\alpha}(M)$.

It is an important fact, due to Aubin, that in a certain sense the Sobolev inequality holds with the same constant on any compact manifold $M$. We present here a simple proof of this result. The technique is typical of the proofs of Theorem 2.2.

TheOREM 2.3 (AUBIN [A1]). Let $M$ be a compact Riemannian manifold with metric $g, p=2 n /(n-2)$, and let $\sigma_{n}$ be the best Sobolev constant defined in Theorem 2.1(a). Then for every $\varepsilon>0$ there exists a constant $C_{\varepsilon}$ such that for all $\varphi \in C^{\infty}(M)$

$$
\|\varphi\|_{p}^{2} \leqslant(1+\varepsilon) \sigma_{n} \int_{M}|\nabla \varphi|^{2} d V_{g}+C_{\varepsilon} \int_{M} \varphi^{2} d V_{g} .
$$

Proof. Fix $\varepsilon>0$. Around each point $P \in M$ we can choose a neighborhood $U$ such that, in normal coordinates on $U$, the eigenvalues of $g_{j k}$ are between $(1+\varepsilon)^{-1}$ and $(1+\varepsilon)$, and furthermore $d V_{g}=f d x$ where $(1+\varepsilon)^{-1}$ $<f<(1+\varepsilon)$. Choose a finite subcover $\left\{U_{i}\right\}$ and a subordinate partition of unity, which we may write as $\left\{\alpha_{i}^{2}\right\}$, where $\alpha_{i} \in C^{\infty}(M)$ and $\sum \alpha_{i}^{2}=1$. Then we have

$$
\begin{aligned}
\|\varphi\|_{p}^{2}=\left\|\varphi^{2}\right\|_{p / 2}=\left\|\sum_{i} \alpha_{i}^{2} \varphi^{2}\right\|_{p / 2} & \leqslant \sum_{i}\left(\int_{M}\left|\alpha_{i} \varphi\right|^{p} d V_{g}\right)^{2 / p} \\
& \leqslant(1+\varepsilon)^{2 / p} \sum_{i}\left(\int_{U_{i}}\left|\alpha_{i} \varphi\right|^{p} d x\right)^{2 / p} .
\end{aligned}
$$


The Sobolev inequality on $\mathbf{R}^{n}$ (2.8), together with our estimates on the deviation of $g$ and $d V_{g}$ from the Euclidean metric, imply

$$
\begin{aligned}
\left(\int_{U_{i}}\left|\alpha_{i} \varphi\right|^{p} d x\right)^{2 / p} & \leqslant \sigma_{n} \int_{U_{i}}\left|\nabla\left(\alpha_{i} \varphi\right)\right|_{0}^{2} d x, \\
& \leqslant(1+\varepsilon)^{2} \sigma_{n} \int_{U_{i}}\left|\nabla\left(\alpha_{i} \varphi\right)\right|^{2} d V_{g},
\end{aligned}
$$

where ||$_{0}$ represents the Euclidean metric in normal coordinates. Furthermore,

$$
\begin{aligned}
\left|\nabla\left(\alpha_{i} \varphi\right)\right|^{2} & =\alpha_{i}^{2}|\nabla \varphi|^{2}+2 \alpha_{i} \varphi\left\langle\nabla \alpha_{i}, \nabla \varphi\right\rangle+\varphi^{2}\left|\nabla \alpha_{i}\right|^{2} \\
& \leqslant(1+\varepsilon) \alpha_{i}^{2}|\nabla \varphi|^{2}+\left(1+\varepsilon^{-1}\right) \varphi^{2}\left|\nabla \alpha_{i}\right|^{2}
\end{aligned}
$$

the last line follows from the Cauchy-Schwartz inequality and the inequality $2 a b<\varepsilon a^{2}+\varepsilon^{-1} b^{2}$. Thus for $\varepsilon$ small,

$$
\begin{aligned}
\|\varphi\|_{p}^{2} & \leqslant(1+4 \varepsilon) \sigma_{n} \sum_{i} \int_{U_{t}} \alpha_{i}^{2}|\nabla \varphi|^{2} d V_{g}+C_{\varepsilon} \sum_{i} \int_{U_{l}} \varphi^{2}\left|\nabla \alpha_{i}\right|^{2} d V_{g} \\
& \leqslant(1+4 \varepsilon) \sigma_{n} \int_{M}|\nabla \varphi|^{2} d V_{g}+C_{\varepsilon}^{\prime} \int_{M} \varphi^{2} d V_{g} .
\end{aligned}
$$

Next we turn to the analysis of the Laplace operator $\Delta$.

TheOREM 2.4 (Local elliptic Regularity). Suppose $\Omega$ is an open set in $\mathbf{R}^{n}$, $\Delta$ is the Laplacian with respect to any metric on $\Omega$, and $u \in L_{\text {loc }}^{1}(\Omega)$ is a weak solution to $\Delta u=f$.

(a) If $f \in L_{k}^{q}(\Omega)$, then $u \in L_{k+2}^{q}(K)$ for any compact set $K \Subset \Omega$, and if $u \in L^{q}(\Omega)$ then

$$
\|u\|_{L_{k+2}^{q}(K)} \leqslant C\left(\|\Delta u\|_{L_{k}^{q}(\Omega)}+\|u\|_{L^{q}(\Omega)}\right) .
$$

(b) (Schauder estimates). If $f \in C^{k, \alpha}(\Omega)$, then $u \in C^{k+2, \alpha}(K)$ for any compact subset $K \Subset \Omega$, and if $u \in C^{\alpha}(\Omega)$ then

$$
\|u\|_{C^{k+2, \alpha}(K)} \leqslant C\left(\|\Delta u\|_{C^{k, \alpha}(\Omega)}+\|u\|_{C^{\alpha}(\Omega)}\right) \text {. }
$$

By a procedure similar to that mentioned above, these results can be transferred to a compact manifold.

THEOREM 2.5 (Global ELliPTIC REGUlaRITY). Let $M$ be a compact Riemannian manifold, and suppose $u \in L_{\mathrm{loc}}^{1}(M)$ is a weak solution to $\Delta u=f$.

(a) If $f \in L_{k}^{q}(M)$, then $u \in L_{k+2}^{q}(M)$, and

$$
\|u\|_{q, k+2} \leqslant C\left(\|\Delta u\|_{q, k}+\|u\|_{q}\right) .
$$

(b) If $\in C^{k, \alpha}(M)$, then $u \in C^{k+2, \alpha}(M)$, and

$$
\|u\|_{C^{k+2, \alpha}} \leqslant C\left(\|\Delta u\|_{C^{k, \alpha}}+\|u\|_{C^{\alpha}}\right) .
$$

TheOrem 2.6 (STRONg MAXIMUM PRINCIPLE). Suppose $h$ is a nonnegative, smooth function on a connected manifold $M$, and $u \in C^{2}(M)$ satisfies $(\Delta+h) u$ $\geqslant 0$. If $u$ attains its minimum $m \leqslant 0$, then $u$ is constant on $M$. 
Proposition 2.7 (WeAK REMOVABle Singularities THEOREM). Let $U$ be an open set in $M$ and $P \in U$. Suppose $u$ is a weak solution of $(\Delta+h) u=0$ on $U-\{P\}$, with $h \in L^{n / 2}(U)$ and $u \in L^{q}(U)$ for some $q>p / 2=n /(n-2)$. Then $u$ satisfies $(\Delta+h) u=0$ weakly on all of $U$.

Proof. We need to show that

$$
\int_{U}(u \Delta \varphi+h u \varphi) d V_{g}=0
$$

for any $\varphi \in C_{c}^{\infty}(U)$. Choose $\alpha \in C_{c}^{\infty}(U)$ with support in a ball $B_{R}(P)$ of small radius $R$ around $P$, such that $\alpha \equiv 1$ in $B_{R / 2}(P)$, and define $\alpha_{\varepsilon}(x)=$ $\alpha(x / \varepsilon)$ in normal coordinates around $P$. Then $\alpha_{\varepsilon}$ is supported in $B_{\varepsilon R}(P)$. Since $\left(1-\alpha_{\varepsilon}\right) \varphi$ is compactly supported in $U-\{P\}$ and $(\Delta+h) u=0$ there,

$$
\int_{U}(u \Delta \varphi+h u \varphi) d V_{g}=\int_{U}\left(u \Delta\left(\alpha_{\varepsilon} \varphi\right)+h u \alpha_{\varepsilon} \varphi\right) d V_{g}
$$

We will show that the right-hand side goes to zero as $\varepsilon \rightarrow 0$.

Note that $h u$ is integrable by Hölder's inequality, and so the second term above goes to zero as the support of $\alpha_{\varepsilon}$ shrinks. As for the first term, we have

$$
\Delta\left(\alpha_{\varepsilon} \varphi\right)=\varphi \Delta \alpha_{\varepsilon}-2\left\langle\nabla \alpha_{\varepsilon}, \nabla \varphi\right\rangle+\alpha_{\varepsilon} \Delta \varphi .
$$

It is easy to check that $\left|\nabla \alpha_{\varepsilon}\right| \leqslant C / \varepsilon$ and $\left|\Delta \alpha_{\varepsilon}\right| \leqslant C / \varepsilon^{2}$. Therefore, if $q^{-1}+r^{-1}$ $=1$,

$$
\begin{aligned}
\left|\int_{U} u \Delta\left(\alpha_{\varepsilon} \varphi\right) d V_{g}\right| & \leqslant C \varepsilon^{-2} \int_{B_{\varepsilon R}}|u| d V_{g} \\
& \leqslant C \varepsilon^{-2}\|u\|_{q}\left(\int_{B_{\varepsilon R}} d V_{g}\right)^{1 / r} \\
& \leqslant C^{\prime} \varepsilon^{-2+n / r}\|u\|_{q} .
\end{aligned}
$$

Since $q>p / 2$ implies $n / r>2$, this goes to zero as $\varepsilon \rightarrow 0$.

Note that the example $u=r^{2-n}$ on Euclidean space shows that the hypothesis $q>p / 2$ cannot be improved.

Theorem 2.8 (Existence of THE Green function). Suppose $M$ is a compact Riemannian manifold of dimension $n \geqslant 3$, and $h$ is a strictly positive smooth function on $M$. For each $P \in M$, there exists a unique smooth function $\Gamma_{P}$ on $M-\{P\}$, called the Green function for $\Delta+h$ at $P$, such that $(\Delta+h) \Gamma_{P}=\delta_{P}$ in the distribution sense, where $\delta_{P}$ is the Dirac measure at $P$.

3. The model case: the sphere. The analysis of the Yamabe equation (1.2) depends upon a precise understanding of the model case of the sphere $S^{n}$. In this section we will describe the solution to the Yamabe problem on $S^{n}$ and prove that the infimum of the Yamabe functional (1.3) is realized by the standard metric on the sphere. We will also show how this leads to the sharp form of the Sobolev inequality on $\mathbf{R}^{n}$. Using the extremals for this inequality we will show that $\lambda(M) \leqslant \lambda\left(S^{n}\right)$ for any compact manifold $M$. 
Let $P=(0, \ldots, 0,1)$ be the north pole on $S^{n} \subset \mathbf{R}^{n+1}$. Stereographic projection $\sigma: S^{n}-\{P\} \rightarrow \mathbf{R}^{n}$ is defined by $\sigma\left(\zeta^{1}, \ldots, \zeta^{n}, \xi\right)=\left(x^{1}, \ldots, x^{n}\right)$ for $(\zeta, \xi)$ $\in S^{n}-\{P\}$, where

$$
x^{j}=\zeta^{j} /(1-\xi) .
$$

It is easy to verify that $\sigma$ is a conformal diffeomorphism. In fact, if $\bar{g}$ is the standard metric on $S^{n}$, and $d s^{2}$ the Euclidean metric on $\mathbf{R}^{n}$, then under $\sigma, \bar{g}$ corresponds to

$$
\rho^{*} \bar{g}=4\left(|x|^{2}+1\right)^{-2} d s^{2},
$$

where $\rho$ denotes $\sigma^{-1}$. This can be written as $4 u_{1}^{p-2} d s^{2}$, where

$$
u_{1}(x)=\left(|x|^{2}+1\right)^{(2-n) / 2} \text {. }
$$

By means of stereographic projection, it is simple to write down conformal diffeomorphisms of the sphere. The group of such diffeomorphisms is generated by the rotations, together with maps of the form $\sigma^{-1} \tau_{v} \sigma$ and $\sigma^{-1} \delta_{\alpha} \sigma$, where $\tau_{v}, \delta_{\alpha}: \mathbf{R}^{n} \rightarrow \mathbf{R}^{n}$ are respectively translation by $v \in \mathbf{R}^{n}$ :

$$
\tau_{v}(x)=x-v,
$$

and dilation by $\alpha>0$;

$$
\delta_{\alpha}(x)=\alpha^{-1} x .
$$

The spherical metric on $\mathbf{R}^{n}$ transforms under dilations to

$$
\delta_{\alpha}^{*} \rho^{*} \bar{g}=4 u_{\alpha}^{p-2} d s^{2}, \quad \text { where } u_{\alpha}(x)=\left(\frac{|x|^{2}+\alpha^{2}}{\alpha}\right)^{(2-n) / 2} .
$$

There is an obvious metric of constant scalar curvature on the sphere, namely the standard metric. It is an important fact that this metric in fact minimizes the Yamabe functional $Q$ (see Theorem 3.2 below). This result is due originally to Aubin [A1], and independently to G. Talenti [Ta]. We will give a simpler proof, due to Karen Uhlenbeck and Morio Obata.

First we note that the infimum $\lambda\left(S^{n}\right)$ is actually attained by a smooth metric $g$ in the conformal class of the standard metric $\bar{g}$. This will be proved later in $\S 4$ (Proposition 4.6). This extremal metric $g$ is thus a metric on $S^{n}$, conformal to the standard one, which has constant scalar curvature. The following proposition shows that such a metric must be the standard one (up to a conformal diffeomorphism and a constant scale factor). Our proof is a simplification of Obata's original argument. (The last step was improved following a suggestion of Roger Penrose.)

Proposition 3.1 (OBata [O]). If $g$ is a metric on $S^{n}$ that is conformal to the standard metric $\bar{g}$ and has constant scalar curvature, then up to a constant scale factor, $g$ is obtained from $\bar{g}$ by a conformal diffeomorphism of the sphere.

Proof. We begin by showing that $g$ is Einstein. Considering the given metric $g$ as "background" metric on the sphere, we can write $\bar{g}=\varphi^{-2} g$, where $\varphi \in C^{\infty}\left(S^{n}\right)$ is strictly positive. Making the substitution $e^{2 f}=\varphi^{-2}$ in the 
transformation law (2.5) for the Ricci tensor, we obtain:

$$
\bar{R}_{j k}=R_{j k}+\varphi^{-1}\left((n-2) \varphi_{j k}-(n-1) \frac{\varphi_{i} \varphi^{i}}{\varphi} g_{j k}-\Delta \varphi g_{j k}\right)
$$

in which the covariant derivatives and Laplacian are to be taken with respect to $g$, not with respect to the standard metric $\bar{g}$. If $B_{j k}=R_{j k}-(S / n) g_{j k}$ represents the traceless Ricci tensor, then since $\bar{g}$ is Einstein,

$$
0=\bar{B}_{j k}=B_{j k}+(n-2) \varphi^{-1}\left(\varphi_{j k}+(1 / n) \Delta \varphi g_{j k}\right) \text {. }
$$

Since the scalar curvature $S$ is constant, the contracted Bianchi identity (2.4) implies that the divergence $R^{i}{ }_{m, i}$ of the Ricci tensor vanishes identically, and thus so also does $B_{m, i}^{i}$. Because $B_{j k}$ is traceless, integration by parts gives

$$
\begin{aligned}
\int_{S^{n}} \varphi|B|^{2} d V_{g} & =\int_{S^{n}} \varphi B_{j k} B^{j k} d V_{g} \\
& =-(n-2) \int_{S^{n}} B^{j k}\left(\varphi_{j k}+\frac{1}{n} \Delta \varphi g_{j k}\right) d V_{g} \\
& =-(n-2) \int_{S^{n}} B^{j k} \varphi_{j k} d V_{g} \\
& =(n-2) \int_{S^{n}} B^{j k}{ }_{, k} \varphi_{j} d V_{g}=0 .
\end{aligned}
$$

Thus $B_{j k}$ must be identically zero, and so $g$ is Einstein.

Since $g$ is conformal to the standard metric $\bar{g}$ on the sphere, which is locally conformally flat, we have $W=0$ as well as $B=0$. As noted in $\S 2$, this implies that $g$ has constant curvature, and so $\left(S^{n}, g\right)$ is isometric to a standard sphere. The isometry is the desired conformal diffeomorphism.

Combining Propositions 3.1 and 4.6, we obtain the complete solution to the Yamabe problem on the sphere:

THEOREM 3.2. The Yamabe functional (1.3) on $\left(S^{n}, \bar{g}\right)$ is minimized by constant multiples of the standard metric and its images under conformal diffeomorphisms. These are the only metrics conformal to the standard one on $S^{n}$ that have constant scalar curvature.

The Sobolev inequality. The above theorem is closely related to the Sobolev inequality (2.8) on $\mathbf{R}^{n}$. Since the infimum of the Yamabe functional on the sphere is conformally invariant, stereographic projection converts the Yamabe problem on $S^{n}$ to an equivalent problem on $\mathbf{R}^{n}$, as follows.

First it is useful to observe that the restriction to smooth positive test functions in the definition (1.5) of $\lambda(M)$ is unnecessary. Indeed, the functional $Q_{g}$ is continuous on $L_{1}^{2}(M)$ by the Sobolev theorem, and $C^{\infty}(M)$ is dense in $L_{1}^{2}(M)$. But $Q_{g}(|\varphi|)=Q_{g}(\varphi)$ for smooth $\varphi$, and a nonnegative function can be approximated arbitrarily closely in $L_{1}^{2}$ norm by a positive function. Thus $\lambda(M)$ is the infimum of $Q_{g}$ over all of $L_{1}^{2}(M)$.

For $\varphi \in C^{\infty}\left(S^{n}\right)$, let $\bar{\varphi}$ denote the weighted push-forward function on $\mathbf{R}^{n}$ defined by $\bar{\varphi}=u_{1} \rho^{*} \varphi$, with $u_{1}$ the conformal factor given by (3.1). We then have

$$
\rho^{*}\left(\varphi^{p-2} \bar{g}\right)=4 \bar{\varphi}^{p-2} d s^{2}
$$


By the conformal invariance of $Q$,

$$
\lambda\left(S^{n}\right)=\inf _{\varphi \in C^{\infty}\left(S^{n}\right)} \frac{\int_{\mathbf{R}^{n}} a|\nabla \bar{\varphi}|^{2} d x}{\left(\int_{\mathbf{R}^{n}}|\bar{\varphi}|^{p} d x\right)^{2 / p}} .
$$

But a simple argument using cutoff functions (cf. Lemma 3.4 below) shows that $\bar{\varphi}$ can be approximated by compactly supported functions, and therefore

$$
\lambda\left(S^{n}\right)=\inf _{\varphi \in C_{0}^{\infty}\left(\mathbf{R}^{n}\right)} a\|\nabla \varphi\|_{2}^{2} /\|\varphi\|_{p}^{2} .
$$

The Sobolev inequality says that $\lambda\left(S^{n}\right)>0$. Thus, identifying $\lambda\left(S^{n}\right)$ and the associated extremal functions is equivalent to identifying the best constant and extremal functions for the Sobolev inequality. Theorem 3.2 then can be rephrased in the following way.

THEOREM 3.3. The $n$-dimensional Sobolev constant $\sigma_{n}$ is equal to a/ $\Lambda$, where

$$
\Lambda=\lambda\left(S^{n}\right)=Q(\bar{g})=n(n-1) \operatorname{vol}\left(S^{n}\right)^{2 / n} .
$$

Thus the sharp form of the Sobolev inequality on $\mathbf{R}^{n}$ is:

$$
\|\varphi\|_{p}^{2} \leqslant \frac{a}{\Lambda} \int_{\mathbf{R}^{n}}|\nabla \varphi|^{2} d x
$$

Equality is attained only by constant multiples and translates of the functions $u_{\alpha}$ defined by (3.2).

An upper bound for the Yamabe invariant. An extremely important feature of the extremal functions $u_{\alpha}$ is that they become more and more concentrated near the origin as $\alpha \rightarrow 0$. In fact, as the following lemma shows, they can be approximated very closely by compactly supported functions, which can in turn be transplanted to a small neighborhood on a manifold $M$ to obtain a test function whose Yamabe quotient is very close to $\lambda\left(S^{n}\right)$.

LEMMA 3.4 (AUBIN [A2]). If $M$ is any compact Riemannian manifold of dimension $n \geqslant 3$, then $\lambda(M) \leqslant \lambda\left(S^{n}\right)$.

Proof. The functions $u_{\alpha}$ satisfy $a\left\|\nabla u_{\alpha}\right\|_{2}^{2}=\Lambda\left\|u_{\alpha}\right\|_{p}^{2}$ on $\mathbf{R}^{n}$. For any fixed $\varepsilon>0$, let $B_{\varepsilon}$ denote the ball of radius $\varepsilon$ in $\mathbf{R}^{n}$, and choose a smooth radial cutoff function $0 \leqslant \eta \leqslant 1$ supported in $B_{2 \varepsilon}$, with $\eta \equiv 1$ on $B_{\varepsilon^{*}}$ Consider the smooth, compactly supported function $\varphi=\eta u_{\alpha}$. Since $\varphi$ is a function of $r=|x|$ alone,

$$
\begin{aligned}
\int_{\mathbf{R}^{n}} a|\nabla \varphi|^{2} d x & =\int_{B_{2 \varepsilon}}\left(a \eta^{2}\left|\nabla u_{\alpha}\right|^{2}+2 a \eta u_{\alpha}\left\langle\nabla \eta, \nabla u_{\alpha}\right\rangle+a u_{\alpha}^{2}|\nabla \eta|^{2}\right) d x \\
& \leqslant \int_{\mathbf{R}^{n}} a\left|\partial_{r} u_{\alpha}\right|^{2} d x+C \int_{A_{\varepsilon}}\left(u_{\alpha}\left|\partial_{r} u_{\alpha}\right|+u_{\alpha}^{2}\right) d x
\end{aligned}
$$


where $A_{\varepsilon}$ denotes the annulus $B_{2 \varepsilon}-B_{\varepsilon}$. To estimate these terms we observe that

$$
\partial_{r} u_{\alpha}=(2-n) r \alpha^{-1}\left(\frac{r^{2}+\alpha^{2}}{\alpha}\right)^{-n / 2}
$$

and so $u_{\alpha} \leqslant \alpha^{(n-2) / 2} r^{2-n}$ and $\left|\partial_{r} u_{\alpha}\right| \leqslant(n-2) \alpha^{(n-2) / 2} r^{1-n}$. Thus, for fixed $\varepsilon$, the second term in (3.7) is $O\left(\alpha^{n-2}\right)$ as $\alpha \rightarrow 0$. For the first term,

$$
\begin{aligned}
\int_{\mathbf{R}^{n}} a\left|\partial_{r} u_{\alpha}\right|^{2} d x & =\Lambda\left(\int_{B_{\varepsilon}} u_{\alpha}^{p} d x+\int_{\mathbf{R}^{n}-B_{\varepsilon}} u_{\alpha}^{p} d x\right)^{2 / p} \\
& \leqslant \Lambda\left(\int_{B_{2 \varepsilon}} \varphi^{p} d x+\int_{\mathbf{R}^{n}-B_{\varepsilon}} \alpha^{n} r^{-2 n} d x\right)^{2 / p} \\
& =\Lambda\left(\int_{B_{2 \varepsilon}} \varphi^{p} d x\right)^{2 / p}+O\left(\alpha^{n}\right) .
\end{aligned}
$$

Therefore the Sobolev quotient of $\varphi$ on $\mathbf{R}^{n}$ is less than $\Lambda+C \alpha^{n-2}$.

On a compact manifold $M$, let $\varphi=\eta u_{\alpha}$ in normal coordinates $\left\{x^{i}\right\}$ in a neighborhood of $P \in M$, extended by zero to a smooth function on $M$. Since $\varphi$ is a radial function and $g^{r r} \equiv 1$ in normal coordinates, we have $|\nabla \varphi|^{2}=\left|\partial_{r} \varphi\right|^{2}$ as before. The only corrections to the above estimate are introduced by the scalar curvature term and the difference between $d V_{g}$ and $d x$. Since $d V_{g}=$ $(1+O(r)) d x$ in normal coordinates, the previous calculation gives

$$
\begin{aligned}
E(\varphi) & =\int_{B_{2 \varepsilon}}\left(a|\nabla \varphi|^{2}+S \varphi^{2}\right) d V_{g} \\
& \leqslant(1+C \varepsilon)\left(\Lambda\|\varphi\|_{p}^{2}+C \alpha^{n-2}+C \int_{0}^{2 \varepsilon} \int_{S_{r}} u_{\alpha}^{2} r^{n-1} d \omega d r\right)
\end{aligned}
$$

Lemma 3.5 below shows that the last term is bounded by a constant multiple of $\alpha$. Thus, choosing first $\varepsilon$ and then $\alpha$ small, we can arrange that

$$
Q_{g}(\varphi) \leqslant(1+C \varepsilon)(\Lambda+C \alpha)
$$

which proves that $\lambda(M) \leqslant \Lambda$.

The following simple calculus lemma will be used again in $\S 5$, so we state it in somewhat more generality than needed for the above argument.

Lemma 3.5. Suppose $k>-n$. Then as $\alpha \rightarrow 0$,

$$
I(\alpha)=\int_{0}^{\varepsilon} r^{k} u_{\alpha}^{2} r^{n-1} d r
$$

is bounded above and below by positive multiples of $\alpha^{k+2}$ if $n>k+4$, $\alpha^{k+2} \log (1 / \alpha)$ if $n=k+4$, and $\alpha^{n-2}$ if $n<k+4$.

Proof. The substitution $\sigma=\mathrm{r} / \alpha$ gives

$$
I(\alpha)=\alpha^{k+2} \int_{0}^{\varepsilon / \alpha} \sigma^{k+n-1}\left(\sigma^{2}+1\right)^{2-n} d \sigma .
$$


Observe that $\sigma^{2} \leqslant \sigma^{2}+1 \leqslant 2 \sigma^{2}$ for $\sigma \geqslant 1$, so $I(\alpha)$ is bounded above and below by positive multiples of

$$
\alpha^{k+2}\left(C+\int_{1}^{\varepsilon / \alpha} \sigma^{k+3-n} d \sigma\right)
$$

The expression in parentheses is bounded if $n>k+4$; it is comparable to $\alpha^{n-k-4}$ if $n<k+4$, and to $\log (1 / \alpha)$ if $n=k+4$.

4. The variational approach. In this section we will prove that the Yamabe problem can be solved on a general compact manifold $M$ provided that $\lambda(M)<\lambda\left(S^{n}\right)$. This is the analytic part of the solution.

The most direct approach to minimizing (1.4) would be to construct a sequence of functions for which the functional $Q_{g}$ approaches its infimum (a "minimizing sequence"), and hope that some subsequence converges to an actual extremal function. However, as we shall see, because the exponent $p$ that occurs in the definition of $Q_{g}$ is exactly the critical exponent for the Sobolev inequality, this direct approach does not work.

Suppose $\left\{u_{i}\right\}$ is a sequence of smooth functions such that $Q_{g}\left(u_{i}\right) \rightarrow \lambda(M)$. By homogeneity we can assume that $\left\|u_{i}\right\|_{p}=1$ for each $i$. Then

$$
\begin{aligned}
\left\|u_{i}\right\|_{2,1}^{2} & =\int_{M}\left(\left|\nabla u_{i}\right|^{2}+u_{i}^{2}\right) d V_{g} \\
& =\frac{1}{a} Q_{g}\left(u_{i}\right)+\int_{M}\left(1-\frac{S}{a}\right) u_{i}^{2} d V_{g} \\
& \leqslant \frac{1}{a} Q_{g}\left(u_{i}\right)+C\left\|u_{i}\right\|_{p}^{2},
\end{aligned}
$$

by Hölder's inequality. Therefore, $\left\{u_{i}\right\}$ is bounded in $L_{1}^{2}(M)$. Since bounded sets in a Hilbert space are weakly precompact, this implies that a subsequence converges weakly to a function $\varphi \in L_{1}^{2}(M)$. But since $p$ is precisely the exponent for which the inclusion $L_{1}^{2} \subset L^{p}$ is not compact, we have no guarantee that the constraint $\left\|u_{i}\right\|_{p}=1$ is preserved in the limit. In particular, the limit function $\varphi$ may be identically zero.

The subcritical equation. Yamabe's approach was to consider first the perturbed functional $Q^{s}(\varphi)=E(\varphi) /\|\varphi\|_{s}^{2}$ for $2 \leqslant s \leqslant p$. We set $\lambda_{s}=\inf \left\{Q^{s}(\varphi)\right.$ : $\left.\varphi \in C^{\infty}(M)\right\}$. Observe that a minimizing function $\varphi$ with $\|\varphi\|_{s}=1$ satisfies

$$
\square \varphi=\lambda_{s} \varphi^{s-1} \text {. }
$$

We will see that, for $s<p$, this equation always has a smooth, positive, minimizing solution $\varphi_{s}$. We begin by showing that elliptic regularity for $\Delta$ implies the following basic regularity result for equation (4.1).

Theorem 4.1 (Regularity Theorem). Suppose $\varphi \in L_{1}^{2}(M)$ is a nonnegative weak solution of (4.1) with $2 \leqslant s \leqslant p$, and $\left|\lambda_{s}\right| \leqslant K$ for some constant $K$. If $\varphi \in L^{r}(M)$ for some $r>(s-2) n / 2$ (in particular if $r=s<p$, or if $s=p<$ $r)$, then $\varphi$ is either identically zero or strictly positive and $C^{\infty}$, and $\|\varphi\|_{C^{2, \alpha}} \leqslant C$, where $C$ depends only on $M, g, K$, and $\|\varphi\|_{r}$. 
Proof. Since $\varphi \in L^{r}(M),(4.1)$ implies that $a \Delta \varphi=\lambda_{s} \varphi^{s-1}-S \varphi \in L^{q}(M)$, with $q=r /(s-1)$. By elliptic regularity (Theorem 2.5), this in turn implies $\varphi \in L_{2}^{q}(M)$. The Sobolev embedding theorem now gives $\varphi \in L^{r^{\prime}}(M)$, with $r^{\prime}=n r /(n s-n-2 r)$. Observe that our hypothesis on $r$ implies $r^{\prime}>r$. Iterating this argument shows that $\varphi \in L_{2}^{q}(M)$ for all $q>1$. Now the $C^{\alpha}$ case of the Sobolev embedding theorem (Theorem 2.2(c)) implies that $\varphi \in C^{\alpha}(M)$ for some $\alpha>0$. Then $\varphi^{s-1} \in C^{\alpha}(M)$ as well, so by elliptic regularity again we conclude that $\varphi \in C^{2, \alpha}(M)$. Each of the above applications of the Sobolev and elliptic regularity theorems gives us a bound on the corresponding norm, so we obtain the desired bound on $\|\varphi\|_{C^{2, \alpha} \text {. }}$

From (4.1) it follows that $(\Delta+m) \varphi \geqslant 0$, for some constant $m \geqslant 0$ and $m \geqslant \sup \left\{\left(S-\lambda_{s} \varphi^{s-2}\right) / a\right\}$. If $\varphi=0$ somewhere, then $\varphi$ is identically zero by the strong maximum principle (Theorem 2.6). Thus $\varphi$ is either strictly positive or zero.

Finally, since $\varphi$ is $C^{2, \alpha}$ and nowhere zero, so is $\varphi^{s-1}$, and so we can apply elliptic regularity iteratively to (4.1) to conclude that $\varphi$ is $C^{\infty}$.

We remark that the above result (without the uniform bound on $\|\varphi\|_{C^{2, \alpha}}$ ) has been proved in the borderline case $r=s=p$ by Trudinger [T]. Since it is not needed for the present argument, we omit it.

Proposition 4.2 (Yamabe [Y]). For $2 \leqslant s<p$, there exists a smooth, positive solution $\varphi_{s}$ to the subcritical equation (4.1), for which $Q^{s}\left(\varphi_{s}\right)=\lambda_{s}$ and $\left\|\varphi_{s}\right\|_{s}=1$.

Proof. Let $\left\{u_{i}\right\} \subset C^{\infty}(M)$ be a minimizing sequence for $Q^{s}$, with $\left\|u_{i}\right\|_{s}=1$. Since $Q^{s}\left(\left|u_{i}\right|\right)=Q^{s}\left(u_{i}\right)$, after replacing $u_{i}$ by $\left|u_{i}\right|$ we may assume $u_{i} \geqslant 0$. As we noted at the beginning of this section, $\left\{u_{i}\right\}$ is bounded in $L_{1}^{2}(M)$. Since the inclusion map $L_{1}^{2} \subset L^{s}$ is compact, a subsequence of the $\left\{u_{i}\right\}$ converges weakly in $L_{1}^{2}$ and strongly in $L^{s}$ to a function $\varphi_{s} \in L_{1}^{2}(M)$ with $\left\|\varphi_{s}\right\|_{s}=1$.

Since by Hölder's inequality the $L^{2}$ norm is dominated by the $L^{s}$ norm, it follows that $\int S u_{i}^{2} \rightarrow \int S \varphi_{s}^{2}$. Weak convergence in $L_{1}^{2}$ implies that

$$
\begin{aligned}
\int_{M}\left|\nabla \varphi_{s}\right|^{2} d V_{g} & =\lim _{i \rightarrow \infty} \int_{M}\left\langle\nabla u_{i}, \nabla \varphi_{s}\right\rangle d V_{g} \\
& \leqslant \limsup _{i \rightarrow \infty}\left(\int_{M}\left|\nabla u_{i}\right|^{2} d V_{g}\right)^{1 / 2}\left(\int_{M}\left|\nabla \varphi_{s}\right|^{2} d V_{g}\right)^{1 / 2}
\end{aligned}
$$

and therefore $Q^{s}\left(\varphi_{s}\right) \leqslant \lim _{i \rightarrow \infty} Q^{s}\left(u_{i}\right)=\lambda_{s}$. But since $\lambda_{s}$ is by definition the infimum of $Q^{s}$, we must have $Q^{s}\left(\varphi_{s}\right)=\lambda_{s}$, and so $\varphi_{s}$ is extremal. Thus $\varphi_{s}$ is a weak solution to the Euler-Lagrange equation (4.1). By the regularity theorem, $\varphi_{s}$ is positive and $C^{\infty}$.

The limit as $s \rightarrow p$. Now our task is to investigate the limit of $\varphi_{s}$ as $s \rightarrow p$. We will see that, provided $\lambda(M)<\lambda\left(S^{n}\right)$, the functions $\varphi_{s}$ converge uniformly to a solution of the Yamabe problem.

We begin by describing the behavior of $\lambda_{s}$. As the following lemma shows, the situation is simplified if the metric $g$ is chosen so that $\int_{M} d V_{g}=1$. From now on, multiplying $g$ by a constant if necessary, we will assume that this holds. We note that Hölder's inequality then implies that $\|u\|_{s} \leqslant\|u\|_{s^{\prime}}$ if $s \leqslant s^{\prime}$. 
LEMMA 4.3 (AUBIN [A2]). If $\int_{M} d V_{g}=1$, then $\left|\lambda_{s}\right|$ is nonincreasing as a function of $s \in[2, p]$; and if $\lambda(M) \geqslant 0, \lambda_{s}$ is continuous from the left.

Proof. We show first that $\left|\lambda_{s}\right|$ is nonincreasing. Observe that for any $s$ and $s^{\prime}$, and any nonzero $u \in C^{\infty}(M)$,

$$
Q^{s^{\prime}}(u)=\frac{\|u\|_{s}^{2}}{\|u\|_{s^{\prime}}^{2}} Q^{s}(u)
$$

If $s \leqslant s^{\prime}$, then $\|u\|_{s} \leqslant\|u\|_{s^{\prime}}$, and therefore $\left|\lambda_{s^{\prime}}\right| \leqslant\left|\lambda_{s}\right|$.

We remark that if $\lambda_{s}<0$ for some $s$, we can choose $u \in C^{\infty}(M)$ such that $Q^{s}(u)<0$, and (4.2) shows that $Q^{s^{\prime}}(u)<0$ for any $s^{\prime}$; thus $\lambda_{s}<0$ for all $s$.

Now suppose $\lambda(M) \geqslant 0$; by the above remark $\lambda_{s} \geqslant 0$ for $2 \leqslant s \leqslant p$. Choose $s \in[2, p]$. Given $\varepsilon>0$, there exists $u \in C^{\infty}(M)$ such that $Q^{s}(u)<$ $\lambda_{s}+\varepsilon$. Since $\|u\|_{s}$ is a continuous function of $s, \lambda_{s^{\prime}} \leqslant Q^{s^{\prime}}(u)<\lambda_{s}+2 \varepsilon$ for $s^{\prime} \leqslant s$ sufficiently close to $s$. Since $\lambda_{s}$ is nonincreasing, this shows that $\lambda_{s}$ is continuous from the left.

The error in Yamabe's proof was the claim that the functions $\left\{\varphi_{s}\right\}$ are uniformly bounded as $s \rightarrow p$. This is false in general, as we will see below in the case of the sphere. However, the following uniform $L^{r}$ bound does hold provided $\lambda(M)<\lambda\left(S^{n}\right)$, and this is sufficient to solve the problem.

It has been traditional to consider separately the cases $\lambda(M) \leqslant 0$ and $\lambda(M)>0$. The following proof disposes of both cases together.

Proposition 4.4 (Trudinger [T], Aubin [A2]). Suppose $\lambda(M)<\lambda\left(S^{n}\right)$, and let $\left\{\varphi_{s}\right\}$ be the collection of functions given by Proposition 4.2. There are constants $s_{0}<p, r>p$, and $C>0$ such that $\left\|\varphi_{s}\right\|_{r} \leqslant C$ for all $s \geqslant s_{0}$.

Proof. Let $\delta>0$. Multiplying (4.1) by $\varphi_{s}^{1+2 \delta}$ and integrating, we obtain

$$
\int_{M}\left(a\left\langle d \varphi_{s},(1+2 \delta) \varphi_{s}^{2 \delta} d \varphi_{s}\right\rangle+S \varphi_{s}^{2+2 \delta}\right) d V_{g}=\lambda_{s} \int_{M} \varphi_{s}^{s+2 \delta} d V_{g}
$$

If we set $w=\varphi_{s}^{1+\delta}$, this can be written

$$
\frac{1+2 \delta}{(1+\delta)^{2}} \int_{M} a|d w|^{2} d V_{g}=\int_{M}\left(\lambda_{s} w^{2} \varphi_{s}^{s-2}-S w^{2}\right) d V_{g}
$$

Now applying the sharp Sobolev inequality (Theorems 2.3 and 3.3), for any $\varepsilon>0$,

$$
\begin{aligned}
\|w\|_{p}^{2} & \leqslant(1+\varepsilon) \frac{a}{\Lambda} \int_{M}|d w|^{2} d V_{g}+C_{\varepsilon} \int_{M} w^{2} d V_{g} \\
& \leqslant(1+\varepsilon) \frac{(1+\delta)^{2}}{(1+2 \delta)} \int_{M} \frac{\lambda_{s}}{\Lambda} w^{2} \varphi_{s}^{s-2} d V_{g}+C_{\varepsilon}^{\prime}\|w\|_{2}^{2} \\
& \leqslant(1+\varepsilon) \frac{(1+\delta)^{2}}{(1+2 \delta)} \frac{\lambda_{s}}{\Lambda}\|w\|_{p}^{2}\left\|\varphi_{s}\right\|_{(s-2) n / 2}^{s-2}+C_{\varepsilon}^{\prime}\|w\|_{2}^{2},
\end{aligned}
$$

by Hölder's inequality. 
Since $s<p,(s-2) n / 2<s$, and thus by Hölder's inequality again $\left\|\varphi_{s}\right\|_{(s-2) n / 2} \leqslant\left\|\varphi_{s}\right\|_{s}=1$. Now if $0 \leqslant \lambda(M)<\Lambda$, then for some $s_{0}<p, \lambda_{s} / \Lambda$ $\leqslant \lambda_{s_{0}} / \Lambda<1$ for $s \geqslant s_{0}$. Thus we can choose $\varepsilon$ and $\delta$ small enough so that the coefficient of the first term above is less than 1 , and so can be absorbed in the left-hand side. Thus

$$
\|w\|_{p}^{2} \leqslant C\|w\|_{2}^{2}
$$

The same result obviously holds if $\lambda(M)$ (and hence $\lambda_{s}$ ) is less than zero. But applying Hölder's inequality once more, we see that

$$
\|w\|_{2}=\left\|\varphi_{s}\right\|_{2(1+\delta)}^{1+\delta} \leqslant\left\|\varphi_{s}\right\|_{s}^{1+\delta}=1
$$

Therefore $\|w\|_{p}=\left\|\varphi_{s}\right\|_{p(1+\delta)}^{1+\delta}$ is bounded independently of $s$.

We can now prove that the Yamabe problem has a solution if $\lambda(M)<\lambda\left(S^{n}\right)$. Theorem $\mathrm{A}$ of the Introduction is an immediate consequence of the following result.

THEOREM 4.5. Let $\left\{\varphi_{s}\right\}$ be the functions given by Proposition 4.2, and assume $\lambda(M)<\lambda\left(S^{n}\right)$. As $s \rightarrow p$, a subsequence converges uniformly to a positive function $\varphi \in C^{\infty}(M)$ which satisfies:

$$
Q_{g}(\varphi)=\lambda(M), \quad \square \varphi=\lambda(M) \varphi^{p-1} .
$$

Thus the metric $\tilde{g}=\varphi^{p-2} g$ has constant scalar curvature $\lambda(M)$.

Proof. Since the functions $\left\{\varphi_{s}\right\}$ are uniformly bounded in $L^{r}(M)$, the regularity theorem shows they are uniformly bounded in $C^{2, \alpha}(M)$ as well. The Arzela-Ascoli theorem then implies that a subsequence converges in $C^{2}$ norm to a function $\varphi \in C^{2}(M)$. The limit function $\varphi$ therefore satisfies

$$
\square \varphi=\lambda \varphi^{p-1}, \quad Q_{g}(\varphi)=\lambda,
$$

where $\lambda=\lim _{s \rightarrow p} \lambda_{s}$. If $\lambda(M) \geqslant 0$, Lemma 4.3 shows that $\lambda=\lambda(M)$. On the other hand, if $\lambda(M)<0$, the fact that $\lambda_{s}$ is increasing implies that $\lambda \leqslant \lambda(M)$; but since $\lambda(M)$ is the infimum of $Q_{g}$ we must have $\lambda=\lambda(M)$ in that case as well.

Now a final application of Theorem 4.1 shows that $\varphi$ is $C^{\infty}$, and it is strictly positive because $\|\varphi\|_{p} \geqslant \lim _{s \rightarrow p}\left\|\varphi_{s}\right\|_{s}=1$.

There are many other methods of obtaining the solution in the case $\lambda(M) \leqslant 0$. Jerry Kazdan [K] has given an excellent survey.

Existence on the sphere. It is important to note that the hypothesis $\lambda(M)<$ $\lambda\left(S^{n}\right)$ is necessary in the previous theorem. On the sphere itself, the presence of a noncompact group of conformal diffeomorphisms defeats the above approach: the family of metrics $\left(\sigma^{-1} \delta_{\alpha} \sigma\right)^{*} \bar{g}$ on the sphere, as $\alpha \rightarrow \infty$, are all solutions to the Yamabe equation, but not uniformly bounded.

On the other hand, the very existence of this family of conformal diffeomorphisms enables us, with a little more effort, to prove existence of extremals on the sphere, by means of the following "renormalization" approach, due to Karen Uhlenbeck. 
Proposition 4.6. There exists a positive, $C^{\infty}$ function $\psi$ on $S^{n}$ satisfying $Q_{\bar{g}}(\psi)=\lambda\left(S^{n}\right)$.

Proof. For $2 \leqslant s<p$, let $\varphi_{s}$ be the solution on $S^{n}$ to the subcritical problem (4.1), given by Proposition 4.2. Composing with a rotation, we may assume that the supremum of $\varphi_{s}$ is attained at the south pole for each $s$. If $\left\{\varphi_{s}\right\}$ is uniformly bounded, the method of Theorem 4.5 shows that a subsequence converges to an extremal solution; so assume from now on that $\sup \varphi_{s} \rightarrow \infty$.

Now let $\kappa_{\alpha}=\sigma^{-1} \delta_{\alpha} \sigma: S^{n} \rightarrow S^{n}$ be the conformal diffeomorphism induced by dilation on $\mathbf{R}^{n}$, as described in the beginning of $\S 3$. If we set $g_{\alpha}=\kappa_{\alpha}^{*} \bar{g}$, we can write $g_{\alpha}=t_{\alpha}^{p-2} \bar{g}$, where the conformal factor $t_{\alpha}$ is the function

$$
t_{\alpha}(\zeta, \xi)=\left(\frac{(1+\xi)+\alpha^{2}(1-\xi)}{2 \alpha}\right)^{(2-n) / 2}
$$

Observe that at the south pole $t_{\alpha}=\alpha^{(2-n) / 2}$.

For each $s<p$, let $\psi_{s}=t_{\alpha} \kappa_{\alpha}^{*} \varphi_{s}$, with $\alpha=\alpha_{s}$ chosen so that $\psi_{s}=1$ at the south pole. This implies that $\alpha_{s}=\left(\sup \varphi_{s}\right)^{2 /(n-2)} \rightarrow \infty$ as $s \rightarrow p$, and $\psi_{s} \leqslant$ $\alpha^{(n-2) / 2} t_{\alpha}$ on $M$. Let $\square_{\alpha}$ denote the conformally invariant Laplacian with respect to the metric $g_{\alpha}$; by naturality of $\square, \square_{\alpha}\left(\kappa_{\alpha}^{*} \varphi_{s}\right)=\kappa_{\alpha}^{*}\left(\square \varphi_{s}\right)$. Then by the transformation law for $\square(2.7)$ we have

$$
\begin{aligned}
\square \psi_{s} & =\square\left(t_{\alpha} \kappa_{\alpha}^{*} \varphi_{s}\right)=t_{\alpha}^{p-1} \square_{\alpha}\left(\kappa_{\alpha}^{*} \varphi_{s}\right)=\lambda_{s} t_{\alpha}^{p-1}\left(\kappa_{\alpha}^{*} \varphi_{s}\right)^{s-1} \\
& =\lambda_{s} t_{\alpha}^{p-s} \psi_{s}^{s-1} .
\end{aligned}
$$

Observe that this transformation law also implies that

$$
\left\|\psi_{s}\right\|_{2,1} \leqslant C \int_{S^{n}} \psi_{s} \square \psi_{s} d V_{\bar{g}}=C \int_{S^{n}} \varphi_{s} \square \varphi_{s} d V_{\bar{g}} \leqslant C^{\prime}\left\|\varphi_{s}\right\|_{2,1}
$$

so $\left\{\psi_{s}\right\}$ is bounded in $L_{1}^{2}\left(S^{n}\right)$, and hence also in $L^{p}\left(S^{n}\right)$ by the Sobolev theorem. Let $\psi \in L_{1}^{2}\left(S^{n}\right)$ denote the weak limit.

Now if $P$ is the north pole, on any compact subset of $S^{n}-\{P\}$ there exists a constant $A$ such that $t_{\alpha} \leqslant A \alpha^{(2-n) / 2}$, and thus the right-hand side of (4.3) is bounded there by $\lambda_{2} A^{p-1}$, independently of $s$. This implies that, on any such set, the right-hand side is bounded in $L^{r}$ for every $r$. Arguing as in the proof of Theorem 4.1, but using local elliptic regularity (Theorem 2.4), $\left\{\psi_{s}\right\}$ is bounded in $C^{2, \alpha}$ on compact sets disjoint from $P$. Let $K_{1} \subset K_{2} \subset \cdots$ be a sequence of compact sets whose union is $S^{n}-\{P\}$. By the Arzela-Ascoli theorem, we can choose a subsequence of $\left\{\psi_{s}\right\}$ that converges in $C^{2}\left(K_{1}\right)$, and then a subsequence that converges in $C^{2}\left(K_{2}\right)$, etc. Taking a diagonal subsequence, we see that the limit function $\psi$ is $C^{2}$ on $S^{n}-\{P\}$.

Since $\lambda_{s} \rightarrow \Lambda$ and $t_{\alpha}^{p-s} \leqslant 1$ away from $P$ for $s$ near $p$, we conclude that $\psi$ satisfies $\square \psi=f \psi^{p-1}$ on $S^{n}-\{P\}$, for some $C^{2}$ function $f$ with $0 \leqslant f \leqslant \Lambda$. By the removable singularities result (Proposition 2.7), the same equation must hold weakly on all of $S^{n}$. For each $s$,

$$
\begin{aligned}
\left\|\psi_{s}\right\|_{p}^{p} & =\int_{S^{n}} t_{\alpha}^{p}\left(\kappa_{\alpha}^{*} \varphi_{s}\right)^{p} d V_{\bar{g}} \\
& =\int_{S^{n}}\left(\kappa_{\alpha}^{*} \varphi_{s}\right)^{p} \kappa_{\alpha}^{*} d V_{\bar{g}}=\left\|\varphi_{s}\right\|_{p}^{p} \geqslant \operatorname{Vol}\left(S^{n}\right)^{1-p / s}\left\|\varphi_{s}\right\|_{s}^{p}
\end{aligned}
$$


This implies that $\|\psi\|_{p} \geqslant 1$, and therefore $Q_{\bar{g}}(\psi) \leqslant \Lambda$. But since $\Lambda$ is by definition the infimum of $Q_{\bar{g}}$, we must have $\square \psi=\Lambda \psi^{p-1}$ and $Q_{\bar{g}}(\psi)=\Lambda$.

It remains to show that $\psi$ is positive and smooth. By the regularity theorem, it suffices to show that $\psi \in L^{r}$ for some $r>p$. For $q>1$ the operator $\square$ : $L_{2}^{q} \rightarrow L^{q}$ has a bounded inverse by elliptic regularity. Consider the perturbation

$$
\square_{\eta}=\square-\eta \Lambda \psi^{p-2}
$$

for $\eta \in C^{\infty}\left(S^{n}\right)$ supported in a small neighborhood of $P . \square_{\eta}$ will also have a bounded inverse if the operator norm of the perturbation term $\eta \Lambda \psi^{p-2}$ is small enough. If we choose $q$ such that $2 n /(n+2)<q<n / 2$ and set $r=n q /(n-2 q)$, then for $u \in L_{2}^{q}$,

$$
\left\|\eta \Lambda \psi^{p-2} u\right\|_{q} \leqslant \Lambda\left\|\eta^{1 /(p-2)} \psi\right\|_{p}^{p-2}\|u\|_{r} \leqslant C \Lambda\left\|\eta^{1 /(p-2)} \psi\right\|_{p}^{p-2}\|u\|_{q, 2},
$$

by the Hölder and Sobolev inequalities. Thus we can make the operator norm as small as we like by choosing $\eta$ with small support and $0 \leqslant \eta \leqslant 1$.

Now $\square_{\eta} \psi=(1-\eta) \Lambda \psi^{p-1} \in L^{q}\left(S^{n}\right)$ since $\psi$ is $C^{2}$ away from $P$. Therefore, since $\square_{\eta}: L_{2}^{q} \rightarrow L^{q}$ is invertible, there exists $\xi \in L_{2}^{q}\left(S^{n}\right) \subset L_{1}^{2}\left(S^{n}\right)$ such that $\square_{\eta}(\xi-\psi)=0$. Using the Sobolev and Hölder inequalities as above, $\|u\|_{2,1}^{2} \leqslant \int u \square u \leqslant \int u \square_{\eta} u+\varepsilon\|u\|_{2,1}^{2}$ when the support of $\eta$ is small. Thus $\square_{\eta}$ is injective on $L_{1}^{2}$, and so we must have $\psi=\xi \in L_{2}^{q}\left(S^{n}\right) \subset L^{r}\left(S^{n}\right)$. Since $r>p$, Theorem 4.1 implies that $\psi$ is $C^{\infty}$, and since $\psi=1$ at the south pole, $\psi$ is strictly positive.

It can be shown, in fact, using methods of $\mathrm{H}$. Brezis, L. Nirenberg, E. Lieb, and P.-L. Lions (see [BL, BN, and L]), that any minimizing sequence on the sphere can be renormalized to converge to a smooth extremal.

Uhlenbeck has adapted the renormalization method described above to give another proof that the Yamabe problem can be solved on any compact manifold $M$ provided that $\lambda(M)<\lambda\left(S^{n}\right)$. Assuming the sequence of solutions to the subcritical equation is not uniformly bounded, she uses Riemannian normal coordinates to transfer the sequence to $\mathbf{R}^{n}$, where a renormalized sequence can be made to converge to a function that contradicts the Sobolev inequality if $\lambda(M)<\lambda\left(S^{n}\right)$. (See also [SU and JL2] for other applications of this technique.)

5. Conformal normal coordinates. In Riemannian geometry, geodesic normal coordinates are invaluable for comparing the local geometry of a manifold with that of $\mathbf{R}^{n}$. In this section we will describe a set of similar coordinate charts on a conformal manifold $M$. These will be normal coordinate charts for some metric $g$ within the conformal class; the freedom in the choice of $g$ will enable us to find coordinate systems that simplify the local geometry more than normal coordinates in a fixed Riemannian structure.

Our first application of conformal normal coordinates will be a very simple proof of Aubin's Theorem B of the Introduction. In the next section we will apply them to the calculation of asymptotic expansions of the Green function for $\square$, and in $\$ 7$ we will use them again to prove Theorem $C$. 
Normalized conformal structures were first constructed by Robin Graham [G], who proved, in connection with his work with Charles Fefferman on conformal invariant theory, that for any $P \in M$ it is possible to find a conformal metric for which the symmetrized covariant derivatives of the Ricci tensor of $g$ vanish at $P$. Later in this section we will give a simple proof of Graham's theorem.

Graham's normalization is designed for simplifying the algebra of conformal invariant theory. For our purposes we want a normalization that simplifies the local analysis. Based on formula (2.1) for the Laplacian, we might guess that a good choice for this normalization would be to choose a conformal metric for which det $g \equiv 1$ in $g$-normal coordinates. (Intrinsically, this means that the Jacobian of the exponential map of $g$ is 1.) This is the normalization we will choose.

THEOREM 5.1 (CONFORMAL NORMAL COORDINATES). Let $M$ be a Riemannian manifold and $P \in M$. For each $N \geqslant 2$ there is a conformal metric $g$ on $M$ such that

$$
\operatorname{det} g_{i j}=1+O\left(r^{N}\right),
$$

where $r=|x|$ in g-normal coordinates at $P$. In these coordinates, if $N \geqslant 5$, the scalar curvature of $g$ satisfies $S=O\left(r^{2}\right)$ and $\Delta S=\frac{1}{6}|W|^{2}$ at $P$.

The proof will be carried out below. First, to illustrate the usefulness of these coordinates, we present an application.

Proof of TheOrem B. Let $\left\{x^{i}\right\}$ be conformal normal coordinates in a neighborhood of $P \in M$. Recalling the notation of Lemma 3.4, let $\varphi=\eta u_{\alpha}$ in $x$-coordinates, where $\eta$ is a cutoff function supported in $B_{2 \varepsilon}$. Since $d V_{g} \equiv d x$ in conformal normal coordinates, the estimates of that lemma apply without the factor $(1+C \varepsilon)$ to show that

$$
E(\varphi) \leqslant \Lambda\|\varphi\|_{p}^{2}+C \alpha^{n-2}+\int_{B_{2 \varepsilon}} S \varphi^{2} d x
$$

But now in conformal normal coordinates $S=O\left(r^{2}\right)$ and $\Delta S(P)$ $=\frac{1}{6}|W(P)|^{2}$, so

$$
\begin{aligned}
\int_{B_{2 \varepsilon}} S \varphi^{2} d x & \leqslant \int_{B_{\varepsilon}} S u_{\alpha}^{2} d x+C \int_{A_{\varepsilon}} u_{\alpha}^{2} d x \\
& =\int_{0}^{\varepsilon} \int_{S_{r}}\left(\frac{1}{2} S_{, i j} x^{i} x^{j}+O\left(r^{3}\right)\right) u_{\alpha}^{2} d \omega_{r} d r+O\left(\alpha^{n-2}\right) \\
& =\int_{0}^{\varepsilon}\left(-C r^{2}|W(P)|^{2}+O\left(r^{3}\right)\right) u_{\alpha}^{2} r^{n-1} d r+O\left(\alpha^{n-2}\right) .
\end{aligned}
$$

Lemma 3.5 therefore shows that

$$
E(\varphi) \leqslant \begin{cases}\Lambda\|\varphi\|_{p}^{2}-C|W(P)|^{2} \alpha^{4}+o\left(\alpha^{4}\right) & \text { if } n>6 \\ \Lambda\|\varphi\|_{p}^{2}-C|W(P)|^{2} \alpha^{4} \log (1 / \alpha)+O\left(\alpha^{4}\right) & \text { if } n=6\end{cases}
$$


If $M$ is not locally conformally flat, we can choose $P$ so that $|W(P)|^{2}>0$, and then $Q_{g}(\varphi)<\Lambda$ for $\alpha$ sufficiently small and $n \geqslant 6$. Thus $\lambda(M)<\Lambda$.

The key ingredient in the proof of Theorem 5.1 is the following inhomogeneous version of Graham's main result.

THEOREM 5.2. Let $P \in M, k \geqslant 0$, and let $T$ be a symmetric $(k+2)$-tensor on $T_{p} M$. There is a unique homogeneous polynomial $f$ of degree $k+2$ in g-normal coordinates such that the metric $\tilde{g}=e^{2 f} g$ satisfies

$$
\operatorname{Sym}\left(\tilde{\nabla}^{k} \tilde{R}_{i j}\right)(P)=T \text {. }
$$

Proof. Let $\left\{x^{i}\right\}$ be $g$-normal coordinates at $P, r=|x|$, and let $\mathscr{P}_{m}$ denote the space of homogeneous polynomials in $x$ of degree $m$. If we set $F_{g}(x)=$ $R_{i j}(x) x^{i} x^{j}$, then by Taylor's theorem

$$
F_{g}=\sum_{m=2}^{k+2} F_{g}^{(m)}+O\left(r^{k+3}\right),
$$

where

$$
F_{g}^{(m)}=\frac{1}{(m-2) !} \sum \partial_{K} R_{i j}(P) x^{i} x^{j} x^{K} \in \mathscr{P}_{m} .
$$

(The sum is over $i, j$ and all multi-indices $K=\left(k_{1}, \ldots, k_{m-2}\right)$ of length $|K|=m-2$.) Observe that the covariant derivatives of $R_{i j}$ are related to ordinary partial derivatives by $R_{i j, K}(P)=\partial_{K} R_{i j}(P)+S_{i j K}$, where the $S_{i j K}$ are constructed as polynomials in the curvature and its derivatives of order $<|K|$ at $P$. If $\tilde{g}=e^{2 f} g$ with $f \in \mathscr{P}_{k+2}$, then $\tilde{S}_{i j K}=S_{i j K}$ when $|K|=k$.

Our result is equivalent to finding $f \in \mathscr{P}_{k+2}$ such that

$$
\begin{aligned}
0 & =\frac{1}{k !} \sum_{|K|=k} \sum_{i, j}\left(\tilde{R}_{i j, K}(P)-T_{i j K}\right) x^{i} x^{j} x^{K} \\
& =F_{\tilde{g}}^{(k+2)}(x)+\sum \frac{1}{k !}\left(S_{i j K}-T_{i j K}\right) x^{i} x^{j} x^{K} .
\end{aligned}
$$

(We have used the fact that $g$-normal coordinates differ from $\tilde{g}$-normal coordinates by $O\left(r^{k+2}\right)$.) By Euler's formula, $x^{i} x^{j} \partial_{i} \partial_{j} f=\left(x^{i} \partial_{i}\right)^{2} f-x^{i} \partial_{i} f=$ $(k+2)(k+1) f$; and $\Delta f=\Delta_{0} f+O\left(r^{k+1}\right)$, where $\Delta_{0}$ is the Euclidean Laplacian in $x$-coordinates. Thus the transformation law (2.5) for $R_{i j}$ gives

$$
\begin{aligned}
F_{\tilde{g}}^{(k+2)}(x) & =F_{g}^{(k+2)}(x)+x^{i} x^{j}\left(-(n-2) \partial_{i} \partial_{j} f+\Delta_{0} f \delta_{i j}\right) \\
& =F_{g}^{(k+2)}(x)-(n-2)(k+2)(k+1) f+r^{2} \Delta_{0} f .
\end{aligned}
$$

Thus there is a unique $f$ so that (5.1) is satisfied provided that the operator $r^{2} \Delta_{0}-(n-2)(k+2)(k+1)$ is invertible on $\mathscr{P}_{k+2}$. This is guaranteed by the following lemma.

LEMMA 5.3. The eigenvalues of $r^{2} \Delta_{0}$ on $\mathscr{P}_{m}$ are

$$
\left\{\lambda_{j}=-2 j(n-2+2 m-2 j): j=0, \ldots,[m / 2]\right\} .
$$


The eigenfunctions corresponding to $\lambda_{j}$ are the functions of the form $r^{2 j} u$, where $u \in \mathscr{P}_{m-2 j}$ is harmonic.

PRoOF. This holds for $m=0$ or 1 since $r^{2} \Delta_{0}=0$ on $\mathscr{P}_{m}$ in these cases. For $m \geqslant 2$ suppose $f \in \mathscr{P}_{m}$ satisfies $r^{2} \Delta_{0} f=\lambda f$. By Euler's formula, $\Delta_{0} f \in \mathscr{P}_{m-2}$ satisfies

$$
\begin{aligned}
\lambda \Delta_{0} f & =\Delta_{0}\left(r^{2} \Delta_{0} f\right)=\Delta_{0}\left(r^{2}\right) \Delta_{0} f-4 x^{i} \partial_{i} \Delta_{0} f+r^{2} \Delta_{0}^{2} f \\
& =-2 n \Delta_{0} f-4(m-2) \Delta_{0} f+r^{2} \Delta_{0}^{2} f,
\end{aligned}
$$

so $r^{2} \Delta_{0}\left(\Delta_{0} f\right)=(\lambda+2 n+4 m-8) \Delta_{0} f$. This means that either $\Delta_{0} f=0$, in which case $\lambda=0$ and $f$ is harmonic, or $\lambda+2 n+4 m-8$ is an eigenvalue of $r^{2} \Delta_{0}$ on $\mathscr{P}_{m-2}$ with eigenfunction $\Delta_{0} f$. In the latter case, $f=\lambda^{-1} r^{2} \Delta_{0} f$. The lemma now follows by induction.

Corollary 5.4 (Graham [G]). Given $P \in M, N \geqslant 0$, there exists a metric conformal to $g$ such that all symmetrized covariant derivatives of the Ricci tensor of order $\leqslant N$ vanish at $P$.

Proof. By induction on $N$ : choose $T=0$ in the above theorem, and note that $f \in \mathscr{P}_{N+2}$ implies that $\tilde{\nabla}^{k} \tilde{R}_{i j}=\nabla^{k} R_{i j}$ for $k<N$.

LEMMA 5.5. In g-normal coordinates, the function det $g_{i j}$ has the expansion

$$
\begin{aligned}
\operatorname{det} g_{i j}= & 1-\frac{1}{3} R_{i j} x^{i} x^{j}-\frac{1}{6} R_{i j, k} x^{i} x^{j} x^{k} \\
& -\left(\frac{1}{20} R_{i j, k l}+\frac{1}{90} R_{p i j m} R_{p k l m}-\frac{1}{18} R_{i j} R_{k l}\right) x^{i} x^{j} x^{k} x^{l}+O\left(r^{5}\right),
\end{aligned}
$$

where the curvatures are evaluated at $P$.

Proof. Let $\left\{x^{i}\right\}$ denote normal coordinates for $g$ on a neighborhood $U$ of $P$, and use them to identify $U$ with an open set in $\mathbf{R}^{n}$. To compute the expansion of the metric $g_{i j}(x)$, we first recall the definition of a Jacobi field.

Fix $\tau, \xi \in \mathbf{R}^{n}$, consider the map $\gamma: \mathbf{R} \times \mathbf{R} \rightarrow \mathbf{R}^{n}$ by $\gamma_{s}(t)=t(\tau+s \xi)$ which gives a one-parameter family of radial geodesics, and let $T=\gamma_{s}^{\prime}(t)$. The variational vector field

$$
X\left(\gamma_{s}(t)\right)=\frac{\partial}{\partial s} \gamma_{s}(t)=t \xi
$$

is called a Jacobi field. Since for each $s, \gamma_{s}$ satisfies the geodesic equation $\nabla_{T} T=0$, and since $0=\gamma_{*}[\partial / \partial t, \partial / \partial s]=[T, X]=\nabla_{T} X-\nabla_{X} T$, we have

$$
\begin{aligned}
0 & =\nabla_{X} \nabla_{T} T=\nabla_{T} \nabla_{X} T-\left(\left[\nabla_{T}, \nabla_{X}\right]-\nabla_{[X, T]}\right) T \\
& =\nabla_{T} \nabla_{T} X-R(T, X) T .
\end{aligned}
$$

Thus $X$ satisfies the Jacobi equation $\nabla_{T}^{2} X=R_{T}(X)$, where $R_{T}$ is the curvature endomorphism $R(T, \cdot) T$.

The Taylor series of $f(t)=\left|X\left(\gamma_{0}(t)\right)\right|^{2}$ can be computed by repeatedly differentiating with respect to $\nabla_{T}$, using the Jacobi equation, and evaluating at $t=0$, noting that $X(0)=0$ and $\nabla_{T} X(0)=\xi$. The first few terms are:

$$
\begin{aligned}
& \nabla_{T} f(0)=0 \text {, } \\
& \nabla_{T}^{2} f(0)=2\langle\xi, \xi\rangle_{0}, \\
& \nabla_{T}^{3} f(0)=0 \text {, } \\
& \nabla_{T}^{4} f(0)=8\left\langle R_{\tau} \xi, \xi\right\rangle_{0} \text {, } \\
& \nabla_{T}^{5} f(0)=20\left\langle\left(\nabla_{\tau} R_{\tau}\right) \xi, \xi\right\rangle_{0} \\
& \nabla_{T}^{6} f(0)=36\left\langle\left(\nabla_{\tau}^{2} R_{\tau}\right) \xi, \xi\right\rangle_{0}+32\left\langle R_{\tau} \xi, R_{\tau} \xi\right\rangle_{0} \text {. }
\end{aligned}
$$


Therefore,

$$
\begin{aligned}
\langle\xi, \xi\rangle_{t \tau}= & t^{-2}\left|X\left(\gamma_{0}(t)\right)\right|^{2} \\
= & \langle\xi, \xi\rangle_{0}+\frac{t^{2}}{3}\left\langle R_{\tau} \xi, \xi\right\rangle_{0}+\frac{t^{3}}{6}\left\langle\left(\nabla_{\tau} R_{\tau}\right) \xi, \xi\right\rangle_{0} \\
& \quad+\frac{t^{4}}{20}\left\langle\left(\nabla_{\tau}^{2} R_{\tau}\right) \xi, \xi\right\rangle_{0}+\frac{2 t^{4}}{45}\left\langle R_{\tau} \xi, R_{\tau} \xi\right\rangle_{0}+O\left(t^{5}\right) .
\end{aligned}
$$

Polarizing this with respect to $\xi$ and substituting $x=t \tau$ yields the expansion for the metric in normal coordinates:

$$
\begin{aligned}
g_{p q}(x)= & \delta_{p q}+\frac{1}{3} R_{p i j q} x^{i} x^{j}+\frac{1}{6} R_{p i j q, k} x^{i} x^{j} x^{k} \\
& +\left(\frac{1}{20} R_{p i j q, k l}+\frac{2}{45} R_{p i j m} R_{q k l m}\right) x^{i} x^{j} x^{k} x^{l}+O\left(r^{5}\right),
\end{aligned}
$$

where the curvature terms are to be evaluated at the origin. This can be written $g_{p q}=\exp A_{p q}$, where

$$
\begin{aligned}
A_{p q}(x)= & \frac{1}{3} R_{p i j q} x^{i} x^{j}+\frac{1}{6} R_{p i j q, k} x^{i} x^{j} x^{k} \\
& +\left(\frac{1}{20} R_{p i j q, k l}-\frac{1}{90} R_{p i j m} R_{q k l m}\right) x^{i} x^{j} x^{k} x^{l}+O\left(r^{5}\right) .
\end{aligned}
$$

Then $\operatorname{det} g_{p q}=\exp \left(\operatorname{tr} A_{p q}\right)$ has the expansion (5.2).

Proof OF TheOREM 5.1. Assume by induction that $g$ satisfies det $g_{i j}=1+$ $O\left(r^{N}\right), N \geqslant 2$. It can easily be seen that each term in the expansion (5.3) for $\langle\xi, \xi\rangle$ has the form

$$
c_{k} t^{k}\left(\left\langle\left(\nabla_{\tau}^{k-2} R_{\tau}\right) \xi, \xi\right\rangle+B_{k}(\xi, \xi)\right)
$$

where $c_{k}$ is a constant and $B_{k}$ is a bilinear form constructed from $R_{\tau}$ and its derivatives of order less than $k-2$. Thus the expansion of $\operatorname{det} g_{i j}$ is of the form

$$
\operatorname{det} g_{i j}=1+\sum_{|K|=N-2} c_{N}\left(R_{i j, K}-T_{i j K}\right) x^{i} x^{j} x^{K}+O\left(r^{N+1}\right),
$$

where $T_{i j K}$ are the coefficients of a symmetric tensor $T$ on $T_{P} M$ constructed from the curvature and its derivatives of order less than $N-2$.

By Theorem 5.2, there is a unique $f \in \mathscr{P}_{N}$ for which $\operatorname{Sym}\left(\tilde{\nabla}^{N-2} \tilde{R}_{i j}\right)=T$. But $T=\tilde{T}$ when $f \in \mathscr{P}_{N}$, so $\operatorname{det} \tilde{g}_{i j}$ vanishes to order $N+1$ in $\tilde{g}$-normal coordinates. From now on, we will replace $g$ by $\tilde{g}$.

The condition det $g_{i j}=1$ means that the symmetrization of the coefficients of (5.2) vanishes, so at $P$

$$
\begin{aligned}
& \text { (a) } 0=R_{i j}, \\
& \text { (b) } 0=R_{i j, k}+R_{j k, i}+R_{k i, j}, \\
& \text { (c) } 0=\operatorname{Sym}\left(R_{i j, k l}+\frac{2}{9} R_{p i j m} R_{p k l m}\right) .
\end{aligned}
$$

Then $R_{i j k l}=W_{i j k l}$ by $(5.5 \mathrm{a})$, and

$$
R_{i j, k l}-R_{i j, l k}=R_{i k l}^{m} R_{m j}+R_{j k l}^{m} R_{i m}=0,
$$


so $(5.5 \mathrm{c})$ gives

$$
\begin{aligned}
0=( & \left.R_{i j, k l}+R_{k l, i j}+2 R_{i k, j l}+2 R_{j l, i k}\right) x^{i} x^{j} \\
+ & \frac{2}{9}\left(W_{p i j m} W_{p k l m}+W_{p i k m} W_{p j l m}+W_{p k i m} W_{p j l m}\right. \\
& \left.+W_{p j k m} W_{p l i m}+W_{p k j m} W_{p l i m}+W_{p l k m} W_{p j i m}\right) x^{i} x^{j} .
\end{aligned}
$$

Now contract on $k, l$, noting the contracted Bianchi identity (2.4), and that $W_{p i k m} W_{p k j m}=\frac{1}{2} W_{p i k m}\left(W_{p k j m}-W_{p m j k}\right)=\frac{1}{2} W_{p i k m} W_{p j k m}$ by the symmetries of the Weyl tensor:

$$
0=\left(3 S_{, i j}+R_{i j, k k}+\frac{2}{3} W_{p i k m} W_{p j k m}\right) x^{i} x^{j} .
$$

Contracting on $i, j$ implies $\Delta S=-S_{, j j}=\frac{1}{6}|W|^{2}$ at $P$.

Finally, $S(P)=R_{j j}(P)=0$ by $(5.5 \mathrm{a}), 0=\left(2 R_{j k, k}+R_{k k, j}\right)(P)=2 S_{, j}(P)$ by (5.5b) and the Bianchi identity, so $S=O\left(r^{2}\right)$.

It is worth noting that, just as Riemannian normal coordinates are unique up to the action of $O(n)$, conformal normal coordinates are unique up to a similar finite-dimensional group action.

TheOREM 5.6. Let $(M, g)$ be a Riemannian manifold and $P \in M$, and let $H$ be the group of conformal diffeomorphisms of $S^{n}$ fixing the north pole. There is a natural simply transitive action of $H$ on the set of formal power series of conformal normal coordinates for the conformal class of $g$ at $P$.

Proof. $H$ is generated by $O(n)$ (rotations fixing the north pole), together with the 'dilations' $\sigma^{-1} \delta_{\alpha} \sigma$ and 'translations' $\sigma^{-1} \tau_{v} \sigma$, as described in $\S 3$. From Theorem 5.2 above, the power series of the metric $\tilde{g}$ with $\operatorname{det} \tilde{g}_{i j}=1$ is uniquely determined once we fix the one-jet of $\tilde{g}$; and conformal normal coordinates are then uniquely determined by the choice of a $\tilde{g}$-orthonormal frame at $P$.

The action of $H$ on the set of conformal normal coordinate charts is obtained as follows: Let $\bar{\sigma}$ be the stereographic projection sending the north pole in $S^{n}$ to $0 \in \mathbf{R}^{n}$. Given conformal normal coordinates $x: M \rightarrow \mathbf{R}^{n}$ for a metric $g$ in the conformal class, and $h \in H$, let $g^{\prime}=\left(x^{-1} \bar{\sigma} h \bar{\sigma}^{-1} x\right)^{*} g$, and let $\left\{e_{i}\right\}$ be the frame obtained by pulling back $\left\{\partial / \partial x^{i}\right\}$ by the same map. It is easy to check that $\left\{e_{i}\right\}$ is $g^{\prime}$-orthonormal and that the one-jet of $g^{\prime}$ is conformal to $g$. So we can let $\left\{y^{i}\right\}$ denote the unique conformal normal coordinates determined by the one-jet of $g^{\prime}$ and the frame $\left\{e_{i}\right\}$, and set $h \cdot x=y$.

Since $O(n)$ acts simply transitively on the set of frames for any choice of metric at $P$, the proof is completed by the observation that $H / O(n)$ acts simply transitively on the set of one-jets of conformal metrics at $P$. (If we write $g^{\prime}=e^{2 f} g$, then $f(0)$ is determined by a dilation, and $d f(0)$ is determined by a translation.)

Of course the above construction of conformal normal coordinates does not achieve the normalization det $g \equiv 1$ exactly; however, we can make the error terms $O\left(r^{N}\right)$ for $N$ arbitrarily large. In the rest of this paper, whenever we refer to conformal normal coordinates, it will be understood that we are calculating modulo such inconsequential error terms. 
Although a formal power series approximation of conformal normal coordinates is sufficient for the Yamabe problem, it would be of interest to know if the normalization can be achieved exactly in a neighborhood of $P$. Such normalized coordinates could be of value in other analytic problems in conformal geometry.

6. Stereographic projections. In $\S 3$ we used stereographic projection to transfer the Yamabe functional from $S^{n}$ to $\mathbf{R}^{n}$, where analysis is simpler. In this section we will describe an analogous conformal map which is defined for any compact manifold $M$ with positive Yamabe invariant. We will then use conformal normal coordinates to compute the asymptotic behavior of this map.

Under stereographic projection, the Euclidean metric pulls back to a metric $\hat{g}$ on $S^{n}-\{P\}$, conformal to the standard metric $\bar{g}$, with zero scalar curvature. Therefore $\hat{g}=G^{p-2} \bar{g}$ for some function $G$ which satisfies $\square G=0$. This function $G$ is singular at $P$ and, in fact, is a multiple of the Green function of $\square$ at $P$ on $S^{n}$. Conversely, if we start with the Green function, then $G^{p-2} \bar{g}$ defines a metric on $S^{n}-\{P\}$ which is isometric to the Euclidean metric on $\mathbf{R}^{n}$. The isometry, of course, is stereographic projection.

The property of $\mathbf{R}^{n}$ that is most useful in the analysis of the Yamabe functional is vanishing scalar curvature. With this in mind, we can repeat the above construction for an arbitrary compact manifold $(M, g)$, replacing $g$ by $G^{p-2} g$, provided the Green function exists. This will always be the case if the Yamabe invariant of $M$ is positive.

Lemma 6.1. Suppose $\lambda(M)>0$. Then at each $P \in M$ the Green function $\Gamma_{P}$ for $\square$ exists and is strictly positive.

Proof. Let $u>0$ be the smooth, positive solution to the subcritical equation (4.1) for any $s<p$ given by Proposition 4.2, and define a new metric $g^{\prime}=u^{p-2} g$. The scalar curvature $S^{\prime}$ of $g^{\prime}$ is $S^{\prime}=u^{1-p} \square u=\lambda_{s} u^{s-p}$. Since $\lambda(M)>0$ implies $\lambda_{s}>0, S^{\prime}$ is strictly positive. Thus by Theorem 2.8 , the Green function $\Gamma_{P}^{\prime}$ for $\square^{\prime}$ exists. If at its minimum $\Gamma_{P}^{\prime} \leqslant 0$, then $\Gamma_{P}^{\prime}$ would be constant by the strong maximum principle, which is impossible; therefore $\Gamma_{P}^{\prime}$ is strictly positive.

Now if we set $\Gamma_{P}(x)=u(P) u(x) \Gamma_{P}^{\prime}(x)$, then $\Gamma_{P}$ is strictly positive, and by (2.7) and Theorem 2.8 it satisfies for any $f \in C_{c}^{\infty}(M)$

$$
\begin{aligned}
u^{-1}(P) f(P) & =\int_{M} \Gamma_{P}^{\prime}(x) \square^{\prime}\left(u^{-1}(x) f(x)\right) d V_{g^{\prime}}(x) \\
& =\int_{M} u^{-1}(P) u^{-1}(x) \Gamma_{P}(x)\left(u^{1-p}(x) \square f(x)\right) u^{p}(x) d V_{g}(x) \\
& =u^{-1}(P) \int_{M} \Gamma_{P}(x) \square f(x) d V_{g}(x) .
\end{aligned}
$$

This is equivalent to $\square \Gamma_{P}=\delta_{P}$. Thus $\Gamma_{P}$ is the Green function for $\square$

If $\lambda(M) \leqslant 0$, the Yamabe problem for $M$ has already been solved by the results of $\S 4$. Thus we will assume throughout this section that $\lambda(M)>0$. In that context we define generalized stereographic projections. 
Definition 6.2. Suppose $(M, g)$ is a compact Riemannian manifold with $\lambda(M)>0$. For $P \in M$ define the metric $\hat{g}=G^{p-2} g$ on $\hat{M}=M-\{P\}$, where

$$
G=(n-2) \omega a \Gamma_{P} .
$$

The manifold $(\hat{M}, \hat{g})$ together with the natural map $\sigma: M-\{P\} \rightarrow \hat{M}$ is called the stereographic projection of $M$ from $P$.

The image manifold of a stereographic projection has a special geometric structure, called asymptotically flat.

Definition 6.3. A Riemannian manifold $N$ with $C^{\infty}$ metric $g$ is called asymptotically flat of order $\tau>0$ if there exists a decomposition $N=N_{0} \cup N_{\infty}$ (with $N_{0}$ compact) and a diffeomorphism $N_{\infty} \leftrightarrow \mathbf{R}^{n}-B_{R}$ for some $R>0$, satisfying:

$$
g_{i j}=\delta_{i j}+O\left(\rho^{-\tau}\right), \quad \partial_{k} g_{i j}=O\left(\rho^{-\tau-1}\right), \quad \partial_{k} \partial_{l} g_{i j}=O\left(\rho^{-\tau-2}\right),
$$

as $\rho=|z| \rightarrow \infty$ in the coordinates $\left\{z^{i}\right\}$ induced on $N_{\infty}$. The coordinates $\left\{z^{i}\right\}$ are called asymptotic coordinates.

This definition apparently depends on the choice of asymptotic coordinates. However, we shall see in $\$ 9$ that the asymptotically flat structure is determined by the metric alone.

Now fix a point $P \in M$. By an initial conformal change we may assume that, near $P, g$ is the metric of a conformal normal coordinate system. In this case we will give a very explicit description of the asymptotically flat structure of the stereographic projection $(\hat{M}, \hat{g})$.

The singularity of the Green function $\Gamma_{P}$ is, to highest order, the same as that of the fundamental solution of the Laplacian on $\mathbf{R}^{n}$. In fact (cf. [PR]), $\Gamma_{P}$ has an asymptotic expansion in terms of the geodesic distance $r$ from $P$, the leading terms of which are readily computed in conformal normal coordinates. For convenience we adopt the following notations.

Notation. We write $f=O^{\prime}\left(r^{k}\right)$ to mean $f=O\left(r^{k}\right)$ and $\nabla f=O\left(r^{k-1}\right)$. $O^{\prime \prime}$ is defined similarly. The set of $C^{\infty}$ functions that vanish to order $k$ at $P$ is denoted $\mathscr{C}_{k}$. As in $\S 5, \mathscr{P}_{k}$ is the space of homogeneous polynomials of degree $k$.

LEMMA 6.4. Let $G$ be given by (6.1). In conformal normal coordinates $\left\{x^{i}\right\}$ at $P, G$ has an asymptotic expansion

$$
G(x)=r^{2-n}\left(1+\sum_{k=4}^{n} \psi_{k}(x)\right)+c \log r+O^{\prime \prime}(1),
$$

where $r=|x|, \psi_{k} \in \mathscr{P}_{k}$, and the $\log$ term appears only if $n$ is even. The leading terms are:

(a) if $n=3,4,5$, or $M$ is conformally flat in a neighborhood of $P$,

$$
G=r^{2-n}+A+O^{\prime \prime}(r) \quad(A=\text { constant }) ;
$$

(b) if $n=6$,

$$
G=r^{2-n}-\frac{1}{288 a}|W(P)|^{2} \log r+O^{\prime \prime}(1)
$$

(c) if $n \geqslant 7$,

$$
\begin{aligned}
G= & r^{2-n}\left[1+\frac{1}{12 a(n-4)}\left(\frac{r^{4}}{12(n-6)}|W(P)|^{2}-S_{, i j}(P) x^{i} x^{j} r^{2}\right)\right] \\
& +O^{\prime \prime}\left(r^{7-n}\right) .
\end{aligned}
$$


This will be proved below. First, we observe that the asymptotically flat structure of $\hat{g}$ can be derived immediately from this lemma.

Let $\left\{x^{i}\right\}$ be conformal normal coordinates on a neighborhood $U$ of $P$, and define 'inverted conformal normal coordinates' $z^{i}=r^{-2} x^{i}$ on $U-\{P\}$. With $\rho=|z|=r^{-1}$ we have

$$
\partial / \partial z^{i}=\rho^{-2}\left(\delta_{i j}-2 \rho^{-2} z^{i} z^{j}\right) \partial / \partial x^{j} .
$$

If we write $\gamma=r^{n-2} G$, the components of $\hat{g}$ in $z$-coordinates are

$$
\begin{aligned}
\hat{g}_{i j}(z) & =\gamma^{p-2} \rho^{4} g\left(\partial / \partial z^{i}, \partial / \partial z^{j}\right) \\
& =\gamma^{p-2}\left(\delta_{i k}-2 \rho^{-2} z^{i} z^{k}\right)\left(\delta_{j l}-2 \rho^{-2} z^{j} z^{l}\right) g_{k l}\left(\rho^{-2} z\right) \\
& =\gamma^{p-2}\left(\delta_{i j}+O^{\prime \prime}\left(\rho^{-2}\right)\right) .
\end{aligned}
$$

If $M$ is conformally flat near $P, g_{k l} \equiv \delta_{k l}$ in conformal normal coordinates, so this reduces to $\hat{g}_{i j}=\gamma^{p-2} \delta_{i j}$ in that case. Noting that the expansions for $G$ give corresponding expansions for $\gamma$, we have proved the following explicit description of the stereographic projection.

THEOREM 6.5. The metric $\hat{g}$ is asymptotically flat of order 1 if $n=3$, order 2 if $n \geqslant 4$ and order $n-2$ if $M$ is conformally flat near $P$. In inverted conformal normal coordinates it has the expansion

$$
\hat{g}_{i j}(z)=\gamma^{p-2}(z)\left(\delta_{i j}+O^{\prime \prime}\left(\rho^{-2}\right)\right)
$$

where, in the three cases of Lemma 6.4,

$$
\begin{aligned}
\gamma(z)= & 1+A \rho^{2-n}+O^{\prime \prime}\left(\rho^{1-n}\right) \quad(A=\text { constant }) ; \\
\gamma(z)= & 1+\frac{1}{288 a}|W(P)|^{2} \rho^{-4} \log \rho+O^{\prime \prime}\left(\rho^{-4}\right) ; \\
\gamma(z)= & 1+\frac{1}{12 a(n-4)} \rho^{-6}\left(\frac{\rho^{2}}{12(n-6)}|W(P)|^{2}-S_{, i j}(P) z^{i} z^{j}\right) \\
& +O^{\prime \prime}\left(\rho^{-5}\right) .
\end{aligned}
$$

Proof of Lemma 6.4. Write $G=r^{2-n}(1+\psi)$. Since $g^{r r}=\operatorname{det} g=1$ in conformal normal coordinates, the Laplacian $\Delta$ (given by $(2.1)$ ) is equal to the Euclidean Laplacian $\Delta_{0}$ when applied to functions of $r$ alone. Hence $\Delta r^{2-n}=$ $\Delta_{0} r^{2-n}=(n-2) \omega \delta_{P}$ on $U$, and the equation $\square G=(n-2) \omega a \delta_{P}$ becomes

$$
\square\left(r^{2-n} \psi\right)+S r^{2-n}=0 .
$$

From (2.1), $\Delta \psi=\Delta_{0} \psi+K \psi$, where

$$
K \psi=\partial_{i}\left(\left(\delta^{i j}-g^{i j}\right) \partial_{j} \psi\right) .
$$

Multiplying by $r^{n} / a$, writing $L=r^{2} \Delta_{0}+2(n-2) r \partial_{r}$, and assuming $\psi$ is continuous, we see that $(6.5)$ is equivalent to

$$
L \psi+\frac{1}{a} r^{2}(S+S \psi+a K \psi)=0 .
$$

We begin by computing a formal asymptotic solution to this equation, writing $\bar{\psi}=\psi_{1}+\cdots+\psi_{n}$, with $\psi_{k} \in \mathscr{P}_{k}$, and solving inductively for each $\psi_{k}$. Since $S=O\left(r^{2}\right)$ in conformal normal coordinates, we start by setting $\psi_{1}=\psi_{2}=\psi_{3}=0$. Suppose by induction we have found $\bar{\psi}=\psi_{1}+\cdots+\psi_{k-1}$ 
such that

$$
L \bar{\psi}+\frac{1}{a} r^{2}(S+S \bar{\psi}+a K \bar{\psi}) \in \mathscr{C}_{k} .
$$

Write the right-hand side as $b_{k}+\mathscr{C}_{k+1}$, with $b_{k} \in \mathscr{P}_{k}$. Now suppose we can find $\psi_{k} \in \mathscr{P}_{k}$ such that

$$
L \psi_{k}+b_{k}=0
$$

Then, noting that $r^{2} S \psi_{k} \in \mathscr{C}_{k+4}$ and $r^{2} K \psi_{k} \in \mathscr{C}_{k+2}$, when we replace $\bar{\psi}$ by $\psi_{1}+\cdots+\psi_{k}$, (6.7) is satisfied with $k$ replaced by $k+1$, completing the induction step.

First consider the case when $n$ is odd. Since $L=r^{2} \Delta_{0}+2 k(n-2)$ on $\mathscr{P}_{k}$, Lemma 5.3 shows that $L$ is always invertible on $\mathscr{P}_{k}$ when $n$ is odd, so we simply take $\psi_{k}=-L^{-1} b_{k}$. By induction, there exists $\bar{\psi}=\psi_{1}+\cdots+\psi_{n}$ such that (6.7) holds with $k=n+1$, which is equivalent to

$$
\square\left(r^{2-n} \bar{\psi}\right)+S r^{2-n} \in r^{-n} \mathscr{C}_{n+1} \text {. }
$$

If $n$ is even, this construction works for $k<n-2$ but then breaks down since $L$ is not invertible on $\mathscr{P}_{k}$ for $k \geqslant n-2$. Observe, however, that $\mathscr{P}_{k}=\operatorname{im} L \oplus \operatorname{ker} L$ since $L$ is self-adjoint with respect to the Euclidean inner product $\left\langle\sum a_{I} x^{I}, \sum b_{J} x^{J}\right\rangle=\sum a_{I} b_{I}$ on $\mathscr{P}_{k}$. When $\operatorname{ker} L \neq 0$, we try instead a function of the form $\psi_{k}=p_{k}+q_{k} \log r$, with $p_{k}, q_{k} \in \mathscr{P}_{k}$. By direct computation,

$$
L\left(p_{k}+q_{k} \log r\right)=L p_{k}+(n-2-2 k) q_{k}+\left(L q_{k}\right) \log r .
$$

Thus if $k \geqslant n-2$ we can solve $L \psi_{k}+b_{k}=0$ with $b_{k} \in \mathscr{P}_{k}$ by writing $-b_{k}=L p_{k}+q_{k}, L q_{k}=0$, and setting

$$
\psi_{k}=p_{k}+(n-2-2 k)^{-1} q_{k} \log r .
$$

For $k<n-2$ the induction proceeds as in the odd-dimensional case. When $k=n-2$, Lemma 5.3 shows that the kernel of $L$ on $\mathscr{P}_{n-2}$ is spanned by $r^{n-2}$. Thus there exist $c \in \mathbf{R}$ and $p_{n-2} \in \mathscr{P}_{n-2}$ such that $\psi_{n-2}=p_{n-2}+$ $c r^{n-2} \log r$ satisfies $L \psi_{n-2}+b_{n-2}=0$.

Before continuing with the next two steps $(k=n-1, n)$ we note that fortunately $\psi_{n-2}$ does not introduce any logarithmic error terms on the right-hand side of (6.7). Indeed, if $\psi$ is a function of $r$ alone then (6.6) and the expansion (5.4) for the metric show that

$$
K \psi=\partial_{i}\left(\left(\frac{1}{3} R_{i k l j} x^{k} x^{l}+\mathscr{C}_{3}\right) r^{-1} x^{j} \partial_{r} \psi\right) .
$$

But $R_{i k l j} x^{k} x^{l} x^{j}=0$ by the symmetries of the curvature, so with $\psi_{n-2}$ as above, $K \psi_{n-2} \in \mathscr{C}_{n-2}+\mathscr{C}_{n-1} \log r$. Writing $\bar{\psi}=\psi_{1}+\cdots+\psi_{n-2}$, this implies

$$
L \bar{\psi}+\frac{1}{a} r^{2}(S+S \bar{\psi}+a K \bar{\psi}) \in \mathscr{C}_{n-1}+\mathscr{C}_{n+1} \log r .
$$

As before, writing the right-hand side as $b_{k}+\mathscr{C}_{k+1}+\mathscr{C}_{n+1} \log r$, we can solve successively for $\psi_{k} \in \mathscr{P}_{k}+\mathscr{P}_{k} \log r, k=n-1, n$. Thus we end up with $\bar{\psi}=\psi_{1}+\cdots+\psi_{n}$ satisfying

$$
\square\left(r^{2-n} \bar{\psi}\right)+S r^{2-n} \in r^{-n} \mathscr{C}_{n+1}+r^{-n} \log r \mathscr{C}_{n+1} .
$$


Now for any $n$ write $\psi=\bar{\psi}+\varphi$. By (6.5) together with (6.8) or (6.10),

$$
\square\left(r^{2-n} \varphi\right)=-\square\left(r^{2-n} \bar{\psi}\right)-S r^{2-n} \in C^{\alpha} .
$$

Therefore by local elliptic regularity $r^{2-n} \varphi \in C^{2, \alpha}$. This proves (6.2), and it remains only to verify the stated expansions of $G$.

If $M$ is conformally flat near $P$, then $S=0$, so (6.5) shows that $r^{2-n} \psi$ is harmonic and hence $C^{\infty}$. Therefore $G=r^{2-n}+A+O^{\prime \prime}(r)$ in that case. If $n=3,4$, or 5 , the same result holds since then $r^{2-n} \varphi \in C^{2, \alpha}$ and $r^{2-n} \bar{\psi}=$ $O^{\prime \prime}(r)$.

Finally, for $n \geqslant 6$, we need only confirm that the leading term $\psi_{4}$ is given by the stated formulas. Expanding $S$ in its Taylor series at $P$, the above proof shows that $\psi_{4}$ satisfies

$$
L \psi_{4}=-(1 / 2 a) r^{2} S_{k l}(P) x^{k} x^{l} .
$$

If $n>6$, beginning with the guess $\psi_{4}=r^{2} b_{k l} x^{k} x^{l}$ and using the fact that $S_{, k k}(P)=-\Delta S(P)=-\frac{1}{6}|W(P)|^{2}$ by Theorem 5.1 , we find by explicit computation that $(6.11)$ is satisfied with

$$
\psi_{4}=\frac{1}{12 a(n-4)}\left(\frac{r^{4}}{12(n-6)}|W(P)|^{2}-S_{, k l}(P) x^{k} x^{l} r^{2}\right) .
$$

If $n=6$, we try $\psi_{4}=r^{2}\left(b_{k l}+c_{k l} \log r\right) x^{k} x^{l}$, and find that

$$
\psi_{4}=-\frac{1}{24 a}\left(S_{, k l}(P) x^{k} x^{l} r^{2}+\frac{r^{4}}{12}|W(P)|^{2} \log r\right)
$$

solves (6.11). This proves the lemma.

This argument can easily be adapted to give a direct proof of the existence of the Green function.

7. The test function estimate. We will now construct a test function on the asymptotically flat manifold $\hat{M}$ and express its Yamabe quotient in terms of a number determined by the geometry of $\hat{M}$. This approach combines the proofs of Theorems $\mathrm{B}$ and $\mathrm{C}$ of the Introduction.

Most of the results in this section were inspired by the work of Schoen [S].

For $\alpha>0$ let $u_{\alpha}$ be the Sobolev extremal functions on $\mathbf{R}^{n}$ given by (3.2). One can check easily that $a \Delta_{0} u_{\alpha}=4 n(n-1) u_{\alpha}^{p-1}$ (where $\Delta_{0}$ is the Euclidean Laplacian), and therefore $\Lambda=\lambda\left(S^{n}\right)=4 n(n-1)\left\|u_{\alpha}\right\|_{p}^{p-2}$.

Fix a large radius $R>0$, let $\rho(z)=|z|$ in inverted conformal normal coordinates (extended to a smooth positive function on $\hat{M}$ ), and let $\hat{M}_{\infty}=$ $\{\rho>R\}$ as in $\S 6$. Define $\varphi$ on $\hat{M}$ by

$$
\varphi(z)= \begin{cases}u_{\alpha}(z) & \rho(z) \geqslant R, \\ u_{\alpha}(R) & \rho(z) \leqslant R,\end{cases}
$$

with $\alpha \gg R$ to be determined later. Observe that, as $\alpha \rightarrow \infty, u_{\alpha}(z)$ becomes very nearly constant for $|z| \leqslant R$, and so we can expect that the effect of replacing $u_{\alpha}$ by a constant inside radius $R$ should become negligible. Moreover, the metric on $\hat{M}_{\infty}$ closely approximates the Euclidean metric, and so the Yamabe quotient $Q_{\hat{g}}(\varphi)$ should become close to $\Lambda$. 
Since $\varphi$ is a function of the radial variable $\rho$ alone, the behavior of $Q_{\hat{g}}(\varphi)$ as $\alpha \rightarrow \infty$ depends on the "average" behavior of the metric $\hat{g}$ over large spheres. It is useful to introduce a number, which we call the 'distortion coefficient' of $\hat{g}$, that measures this average behavior.

It is well known that the scalar curvature measures the deviation of volumes from the Euclidean case. To see this, let $\tilde{g}$ denote any metric on a manifold $M$, and let $r=|x|$ in normal coordinates around a point $P \in M$. The ratio of the $\tilde{g}$-volume of the geodesic sphere $S_{r}$ around $P$ to its Euclidean volume is given by the spherical density function

$$
h(r)=\omega_{r}^{-1} \int_{S_{r}} d \tilde{\omega}_{r},
$$

where $d \tilde{\omega}_{r}$ is the volume element induced on $S_{r}$ by $\tilde{g}$. Since the unit normal vector to $S_{r}$ is $N=\operatorname{grad} r /|\operatorname{grad} r|$, we have

$$
\left.\left.d \tilde{\omega}_{r}=N\right\lrcorner d V_{\tilde{g}}=(\operatorname{det} \tilde{g})^{1 / 2}\left(\tilde{g}^{r r}\right)^{-1 / 2} r^{-1} \tilde{g}^{i j} x^{j} \partial_{i}\right\lrcorner d x .
$$

But since $\left.\partial_{i}\right\lrcorner d x=r^{-1} x^{i} d \omega_{r}$ on $S_{r}$, this reduces to $d \tilde{\omega}_{r}=\left(\tilde{g}^{r r} \operatorname{det} \tilde{g}\right)^{1 / 2} d \omega_{r}$. Thus

$$
h(r)=\omega_{r}^{-1} \int_{S_{r}}\left(\tilde{g}^{r r} \operatorname{det} \tilde{g}\right)^{1 / 2} d \omega_{r}
$$

Since $\tilde{g}^{r r} \equiv 1$ in normal coordinates at $P$, the expansion (5.2) for $\operatorname{det} \tilde{g}$ shows that

$$
h(r)=\omega_{r}^{-1} \int_{S_{r}}\left(1-\frac{1}{6} R_{i j} x^{i} x^{j}+O\left(r^{3}\right)\right) d \omega_{r}=1-\frac{1}{6 n} S r^{2}+O\left(r^{3}\right) .
$$

Thus when the scalar curvature $S(P)$ is positive, volumes of geodesic spheres grow slower than in $\mathbf{R}^{n}$, as on the sphere, and when $S(P)<0$ they grow faster, as in hyperbolic space. (The scalar curvature has a similar meaning using balls rather than spheres. In dimension 2, this is a familiar interpretation of Gaussian curvature.)

On an asymptotically flat manifold we will consider this same function $h(\rho)$ for large values of $\rho=|z|$. In particular, on the manifold $(\hat{M}, \hat{g})$ obtained in $\$ 6$ by stereographic projection with inverted conformal normal coordinates $\left\{z^{i}\right\}$, we have $\hat{g}^{\rho \rho}=\gamma^{2-p}$ and $\operatorname{det} \hat{g}=\gamma^{2 p}$. Thus (7.1) reduces to

$$
h(\rho)=\omega_{\rho}^{-1} \int_{S_{\rho}} \gamma^{(p+2) / 2} d \omega_{\rho}
$$

The expansion of $\gamma$ given by Theorem 6.5 then gives an asymptotic expansion as $\rho \rightarrow \infty$ :

$$
h(\rho)= \begin{cases}1+(\mu / k) \rho^{-k}+O^{\prime \prime}\left(\rho^{-k-1}\right) & \text { if } n \neq 6 \\ 1+(\mu / 4) \rho^{-4} \log \rho+O^{\prime \prime}\left(\rho^{-4}\right) & \text { if } n=6\end{cases}
$$


and therefore, since the $(n-1)$-form $d \omega_{\rho} / \omega_{\rho}$ is homogeneous of degree zero,

$$
\begin{aligned}
\frac{a}{2} \int_{S_{\rho}} \partial_{\rho} \gamma \frac{d \omega_{\rho}}{\omega_{\rho}} & =h^{\prime}(\rho)+O\left(\rho^{-2 k-1}\right) \\
& = \begin{cases}-\mu \rho^{-k-1}+O\left(\rho^{-k-2}\right) & \text { if } n \neq 6 \\
-\mu \rho^{-5} \log \rho+O\left(\rho^{-5}\right) & \text { if } n=6 .\end{cases}
\end{aligned}
$$

We call the constant $\mu$, defined using inverted conformal normal coordinates, the distortion coefficient of $\hat{g}$. Its geometric meaning at infinity is analogous to that of the scalar curvature at a finite point. It is this constant that determines the values of $Q_{\hat{g}}(\varphi)$ for large $\alpha$.

Proposition 7.1. Let $\varphi$ be defined as above. There are positive constants $C$ and $k$ such that

$$
E(\varphi) \leqslant \Lambda\|\varphi\|_{p}^{2}-C \mu \alpha^{-k}+O\left(\alpha^{-k-1}\right)
$$

if $n \neq 6$ or $M$ is conformally flat near $P$,

$$
E(\varphi) \leqslant \Lambda\|\varphi\|_{p}^{2}-C \mu \alpha^{-4} \log \alpha+O\left(\alpha^{-4}\right)
$$

if $n=6$ and $M$ is not conformally flat near $P$.

Thus if $\mu>0, \varphi$ can be chosen so that $Q_{\hat{g}}(\varphi)<\Lambda$.

Proof. Since the scalar curvature of $\hat{g}$ is zero, the energy $E(\varphi)$ is

$$
E(\varphi)=\int_{\hat{M}} a|\nabla \varphi|^{2} d V_{\hat{g}}=\int_{\hat{M}_{\infty}} a \hat{g}^{\rho \rho}\left(\partial_{\rho} u_{\alpha}\right)^{2} d V_{\hat{g}}=\int_{\hat{M}_{\infty}} a\left(\partial_{\rho} u_{\alpha}\right)^{2} \gamma^{2} d z
$$

Letting $A_{L}$ denote the annulus $\{R \leqslant \rho \leqslant L\}$ and integrating by parts using the Euclidean Laplacian gives

$$
\begin{aligned}
& \int_{A_{L}} a\left(\partial_{\rho} u_{\alpha}\right)^{2} \gamma^{2} d z \\
& \left.=\int_{A_{L}} a u_{\alpha} \Delta_{0} u_{\alpha} \gamma^{2} d z-\int_{A_{L}} a u_{\alpha} \partial_{\rho} u_{\alpha} \partial_{\rho}\left(\gamma^{2}\right) d z-\int_{S_{R} \cup S_{L}} a u_{\alpha} \partial_{\rho} u_{\alpha} \gamma^{2} \partial_{\rho}\right\lrcorner d z .
\end{aligned}
$$

Since $\gamma$ is bounded, (3.8) shows that the integral over $S_{L}$ is $O\left(L^{2-n}\right)$ for fixed $\alpha$, and thus vanishes as $L \rightarrow \infty$. Similarly, the integral over $S_{R}$ is $O\left(\alpha^{-n}\right)$. We can bound the first integral by using Hölder's inequality and formula (3.2) for $u_{\alpha}$ :

$$
\begin{aligned}
\int_{A_{L}} a u_{\alpha} \Delta_{0} u_{\alpha} \gamma^{2} d z & =4 n(n-1) \int_{A_{L}} u_{\alpha}^{p-2}\left(u_{\alpha} \gamma\right)^{2} d z \\
& \leqslant 4 n(n-1)\left(\int_{A_{L}} u_{\alpha}^{p} d z\right)^{1-2 / p}\left(\int_{A_{L}} u_{\alpha}^{p} \gamma^{p} d z\right)^{2 / p} \\
& \leqslant 4 n(n-1)\left\|u_{\alpha}\right\|_{p}^{p-2}\left(\int_{\hat{M}} \varphi^{p} d V_{\hat{g}}\right)^{2 / p} \\
& =\Lambda\|\varphi\|_{p}^{2} .
\end{aligned}
$$


The critical term is the second term in (7.7). After letting $L \rightarrow \infty$ it becomes

$$
-\int_{R}^{\infty} a u_{\alpha} \partial_{\rho} u_{\alpha} \int_{S_{\rho}} \partial_{\rho}\left(\gamma^{2}\right) d \omega_{\rho} d \rho .
$$

From (7.4), if $n \neq 6$ or $M$ is conformally flat near $P$,

$$
\begin{aligned}
a \int_{S_{\rho}} \partial_{\rho}\left(\gamma^{2}\right) d \omega_{\rho} & =4\left(h^{\prime}(\rho)+O\left(\rho^{-2 k-1}\right)\right) \omega_{\rho} \\
& =-4\left(\mu \rho^{-k-1}+O\left(\rho^{-k-2}\right)\right) \omega_{\rho} .
\end{aligned}
$$

Just as in Lemma 3.5, the change of variables $\sigma=\rho / \alpha$ shows that if $2-n<k$ $<n$

$$
C^{-1} \alpha^{-k+1} \leqslant \int_{R}^{\infty} \rho^{-k}\left(\frac{\rho^{2}+\alpha^{2}}{\alpha}\right)^{1-n} \rho^{n-1} d \rho \leqslant C \alpha^{-k+1} .
$$

Thus the second term in (7.7) is

$$
\begin{gathered}
-4 \int_{R}^{\infty} \rho \alpha^{-1}\left(\frac{\rho^{2}+\alpha^{2}}{\alpha}\right)^{1-n}\left(\mu \rho^{-k-1}+O\left(\rho^{-k-2}\right)\right) \omega_{\rho} d r \\
\leqslant-C \mu \alpha^{-k}+O\left(\alpha^{-k-1}\right) .
\end{gathered}
$$

Combining the results of the above calculations, we obtain (7.5). If $n=6$, we use instead the inequality

$$
C^{-1} \alpha^{-k+1} \log \alpha \leqslant \int_{R}^{\infty} \rho^{-k} \log \rho\left(\frac{\rho^{2}+\alpha^{2}}{\alpha}\right)^{1-n} \rho^{n-1} d \rho \leqslant C \alpha^{-k+1} \log \alpha,
$$

and a similar analysis yields (7.6).

The above calculation reduces the solution of the Yamabe problem in the case $\lambda(M)>0$ to determining the sign of $\mu$. Indeed, by the same argument as we used in the case of $\mathbf{R}^{n}$ to prove (3.5), we have

$$
\lambda(M)=\inf _{\psi \in C_{0}^{\infty}(\hat{M})} \frac{E(\psi)}{\|\psi\|_{p}^{2}},
$$

and so approximating our test function $\varphi$ by a function $\psi \in C_{0}^{\infty}(\hat{M})$, we find that $\lambda(M)<\lambda\left(S^{n}\right)$ if $\mu>0$. So we have proved the following theorem.

THEOREM 7.2. If $(M, g)$ is a compact Riemannian manifold of dimension $n \geqslant 3$ with $\lambda(M)>0$, then $\lambda(M)<\lambda\left(S^{n}\right)$ if there is a generalized stereographic projection $\hat{M}$ of $M$ with strictly positive distortion coefficient $\mu$.

The distortion coefficient, defined by (7.2) and (7.3), is obtained from the first correction term in the asymptotic expansion of $\gamma$. If $n \geqslant 6$ and $M$ is not locally conformally flat, Theorem 6.5 gives this term explicitly in terms of local geometric invariants of $M$. In the remaining cases, however, it turns out that $\mu$ can be identified with a global invariant called the 'mass' of the asymptotically flat manifold $\hat{M}$. The next three sections are devoted to a detailed study of this invariant. 
8. General relativity. Asymptotically flat manifolds were originally studied by physicists. In this section we will describe how they arise in general relativity, and how physical reasoning leads to the identification of a fundamental geometric invariant called the mass.

General relativity models the world by a four-dimensional spacetime manifold $X$ with a Lorentz metric $g$. The metric represents the gravitational field, and as such it plays two roles in the theory. First, the metric determines the dynamics: the trajectories ("worldlines") of freely falling point particles are geodesics. Second, the metric itself satisfies an equation that describes how a matter distribution determines its gravitational field. This equation, the Einstein field equation, is

$$
R_{i j}-\frac{1}{2} S g_{i j}=T_{i j},
$$

in which the metric tensor $g$ plays a role analogous to the classical gravitational potential, and the energy-momentum tensor $T$ is analogous to the classical mass density.

The most famous solution of this equation is the Schwarzschild metric, which represents the gravitational field of a static point particle (a "black hole") of mass $m$. It is a (singular) Lorentz metric on $\mathbf{R}^{4}$ which, when restricted to any constant-time three-plane, is asymptotically flat of order 1 and has the form

$$
g_{i j}(z)=\left(1+m \rho^{-1}\right) \delta_{i j}+O\left(\rho^{-2}\right)
$$

in suitable coordinates.

More realistic solutions of (8.1) model isolated gravitational systems (e.g. a binary star in an otherwise empty universe). Physically, one expects that when such a system is observed from a great distance its gravitational field should resemble that of a point mass. Thus the spacetime $(X, g)$ modeling the system should be asymptotically Schwarzschild, and should admit spacelike hypersurfaces which are asymptotically flat Riemannian 3-manifolds.

It is in this context that physicists began to study solutions of (8.1) on asymptotically flat manifolds. One way to do this is to introduce the EinsteinHilbert action integral:

$$
A(g)=-\int_{X} S_{g} d V_{g}
$$

The first variation of $A(g)$ gives the vacuum Einstein equation, as shown by the following well-known lemma.

LEMMA 8.1. Given a Riemannian or pseudo-Riemannian manifold $(X, g)$, let $h$ be a smooth symmetric 2-tensor, and consider a one-parameter family of metrics $g_{t}$ with $h=d g_{t} / d t$ at $t=0$. If $S_{t}$ and $d V_{t}$ denote the scalar curvature and volume form of $g_{t}$, then

$$
\frac{d}{d t}\left(S_{t} d V_{t}\right)_{t=0}=-\left(h^{j k} G_{j k}+\nabla^{*} \xi\right) d V_{g},
$$

where $G$ is the Einstein tensor $G_{j k}=R_{j k}-\frac{1}{2} S g_{j k}$, and $\xi$ is the 1-form

$$
\xi=-\left(\nabla^{*} h+\nabla\left(\operatorname{tr}_{g} h\right)\right)=\left(h_{j k}{ }^{k}-h_{k, j}^{k}\right) d x^{j} .
$$


Proof. In any local coordinate system, the variation of the volume form $d V_{t}=\sqrt{\operatorname{det} g_{t}} d x$ is

$$
\left.\frac{d}{d t} d V_{t}\right|_{t=0}=\frac{1}{2} \sqrt{\operatorname{det} g} g^{j k} h_{j k} d x=\frac{1}{2} h^{j k} g_{j k} d V_{g} .
$$

The classical expression for the Ricci tensor is

$$
R_{j k}=\partial_{i} \Gamma_{k j}^{i}-\partial_{k} \Gamma_{i j}^{i}+\Gamma_{i l}^{i} \Gamma_{j k}^{l}-\Gamma_{k l}^{i} \Gamma_{i j}^{l} .
$$

At the origin in $g$-normal coordinates (where $\partial_{i} g_{j k}=0$ ) we have

$$
\partial_{l} \Gamma_{i j}^{k}=\frac{1}{2} g^{k m} \partial_{l}\left(\partial_{i} g_{j m}+\partial_{j} g_{i m}-\partial_{m} g_{i j}\right) \text {. }
$$

Thus, computing $d S_{t} / d t$ and evaluating at the origin yields

$$
\begin{aligned}
\left.\frac{d}{d t} S_{t}\right|_{t=0} & =\frac{d}{d t}\left[g_{t}^{j k}\left(R_{j k}\right)_{t}\right]_{t=0} \\
& =-2 h^{j k} R_{j k}+g^{j k} g^{i l} \partial_{k}\left(\partial_{l} h_{i j}-\partial_{j} h_{i l}\right) .
\end{aligned}
$$

From the definition of covariant differentiation,

$$
h_{j k, l}=\partial_{l} h_{j k}-\Gamma_{k l}^{m} h_{j m}-\Gamma_{j l}^{m} h_{m k},
$$

and thus if $\xi$ is defined by (8.5), at the origin in normal coordinates we have

$$
\begin{aligned}
-\nabla^{*} \xi & =h_{j k},^{k j}-h_{k, j}^{k} \\
& =\partial_{j} \partial_{k} h_{j k}-\partial_{j} \partial_{j} h_{k k}+\left(\partial_{k} \Gamma_{j k}^{m}-\partial_{j} \Gamma_{k k}^{m}\right) h_{j m} \\
& =\partial_{j} \partial_{k} h_{j k}-\partial_{j} \partial_{j} h_{k k}-R_{j m} h_{j m},
\end{aligned}
$$

where we have used the fact that $R_{j m}=-R_{j k m}^{k}$ by the symmetries of the curvature tensor. Thus we have the tensorial identity

$$
\left.\frac{d}{d t} S_{t}\right|_{t=0}=-h^{j k} R_{j k}-\nabla^{*} \xi
$$

Combining this with (8.6) yields the result.

Physicists apply this lemma with compactly supported variations. If $h$ is compactly supported, the lemma and the divergence theorem show that at $t=0$

$$
\frac{d}{d t} A\left(g_{t}\right)=\int_{X} h^{j k} G_{j k} d V_{g},
$$

and thus a Lorentz metric $g$ is a critical point of $A$ if it satisfies the vacuum Einstein equation $G_{i j} \equiv 0$.

The variational lemma can also be applied on an asymptotically flat Riemannian manifold $(N, g)$. We can then look for metrics that are critical for $A(g)$ under all variations that maintain the asymptotically flat structure (not just compactly supported ones). The divergence term in the lemma then comes into play. If we integrate (8.4) over a large sphere $S_{R}$ in the asymptotic end and take the limit as $R \rightarrow \infty$, we obtain

$$
\left.\frac{d}{d t} A\left(g_{t}\right)\right|_{t=0}=\int_{N} h^{j k} G_{j k} d V_{g}-\lim _{R \rightarrow \infty} \int_{S_{R}} \xi(N) d V_{g}
$$

where $\xi_{j}=\left(\partial_{i} h_{i j}-\partial_{j} h_{i i}\right)\left(1+O\left(\rho^{-1}\right)\right)$. One sees immediately that this boundary term is the variation of a geometric invariant called the mass. 
DEFINITION 8.2. Given an asymptotically flat Riemannian manifold $(N, g)$ with asymptotic coordinates $\left\{z^{i}\right\}$, define the mass as follows:

$$
\left.m(g)=\lim _{R \rightarrow \infty} \omega^{-1} \int_{S_{R}} \mu\right\lrcorner d z,
$$

if the limit exists, where $\mu$ is the mass-density vector field defined on $N_{\infty}$ :

$$
\mu=\left(\partial_{i} g_{i j}-\partial_{j} g_{i i}\right) \partial_{j} .
$$

The preceding discussion shows that for a family $g_{t}$ of asymptotically flat metrics,

$$
\frac{d}{d t}\left(A\left(g_{t}\right)+m\left(g_{t}\right)\right)_{t=0}=\int_{N} h^{j k} G_{j k} d V_{g},
$$

provided the mass is a differentiable function of $g$. We will discuss the analytic properties of the mass, including the justification for this equation, in the next section. For now we return to the physics, describing how the mass occurs in general relativity and why the term "mass" is appropriate.

In 1960, Arnowitt, Deser, and Misner [ADM1, 2, 3] made a detailed study of isolated gravitational systems. They adopted the Hamiltonian viewpoint, which meant choosing a spacelike hypersurface as an "initial surface" and writing Einstein's equations as evolution equations from this initial data. After integrating by parts, they discovered a conserved quantity given precisely by the integral (8.10). Using equation (8.2) it is easy to check that in Schwarzschild space $m(g)$ agrees with the mass $m$ (up to a constant). Thus, they concluded, the conserved quantity $m(g)$ is the total mass of the isolated system.

Arnowitt, Deser, and Misner then conjectured that the mass, measured along a spacelike hypersurface in a physical spacetime, is nonnegative (and zero only if the spacetime is empty). Now the metric of any physical spacetime must satisfy Einstein's equation (8.1), where $T_{i j}$ is a physically reasonable energymomentum tensor. It turns out that the energy-momentum tensors encountered in realistic physical models satisfy a certain positivity condition, called the dominant energy condition (see [HE]). By Einstein's equation this becomes a positivity condition on the Ricci tensor. In particular, for a time-independent spacetime $X=N^{3} \times \mathbf{R}$ this condition is equivalent to the requirement that the scalar curvature of $N$ (which then represents local mass density) be nonnegative. Thus one case of the ADM conjecture is:

PoSITIVE MASS CONJECTURE. If $(N, g)$ is an asymptotically flat Riemannian 3-manifold with nonnegative scalar curvature then $m(g) \geqslant 0$, with equality if and only if $(N, g)$ is isometric to $\mathbf{R}^{3}$ with its Euclidean metric.

In fact, Arnowitt, Deser, and Misner found that the mass is the first component of a conserved 4-vector, which represents the total energy-momentum of the system. If the system, viewed from a large distance, behaves like a relativistic point particle then this energy-momentum vector should be timelike. The general form of their conjecture, called the positive energy conjecture, is that this is the case in any spacetime satisfying the dominant energy condition (see [W] or [PT] for a precise statement). This general conjecture is essentially equivalent to the positive mass conjecture (see [SY3]). 
The mass integral (8.10) on a 4-dimensional Riemannian manifold also appears in physics. In his "Euclidean" approach to quantum gravity, Stephen Hawking proposed that one replace the spacetime manifold by an asymptotically flat Riemannian 4-manifold, and quantize its metric. (This rather unintuitive substitution has proved quite valuable in studying Yang-Mills theories.) One finds that the Einstein-Hilbert action $A(g)$ must be replaced by

$$
B(g)=A(g)+m(g) .
$$

Equation (8.11) then shows that the critical points of $B$, thought of as a functional on the space of asymptotically flat metrics, satisfy $G_{j k} \equiv 0$. Gibbons, Hawking, and Perry [GHP] analyzed this action $B(g)$ and concluded that it might lead to a reasonably well-behaved quantum gravitational theory provided $B(g) \geqslant 0$ whenever $g$ has zero scalar curvature. This led them to the following conjecture.

Positive ACTION CONJECTURE. If $(N, g)$ is an asymptotically flat Riemannian 4-manifold with zero scalar curvature then $B(g) \geqslant 0$, with equality if and only if $(N, g)$ is isometric to $\mathbf{R}^{4}$ with its Euclidean metric.

Note that this would be an easy consequence of the 4-dimensional analogue of the positive mass conjecture.

These conjectures have a long history. Partial results were obtained by numerous physicists and mathematicians over a 20-year period (see [W] for references). Finally, in 1979 Richard Schoen and S.-T. Yau solved the problem. In a series of papers [SY1, SY2, SY3, SY4] they used geometric and p.d.e. methods to give complete, rigorous proofs to all of these conjectures. Shortly thereafter Edward Witten gave a simpler proof of the positive energy theorem based on an integration-by-parts formula for spinors [W, PT].

While these conjectures arose in general relativity, they are purely geometric facts about asymptotically flat manifolds. The natural generalization of both conjectures is that an asymptotically flat Riemannian manifold $(N, g)$ of dimension $n \geqslant 3$ with nonnegative scalar curvature has $m(g) \geqslant 0$, with equality if and only if $N$ is isometric to Euclidean $\mathbf{R}^{n}$. Schoen and Yau [S] have announced a proof of this result, which we will continue to call the positive mass theorem.

9. Analysis on asymptotically flat manifolds. Before we sketch the proof of the general positive mass theorem, we pause to introduce some analytic tools that will be useful for understanding asymptotically flat manifolds, analogous to those discussed in $\S 2$ for compact manifolds.

The appropriate analytic setting for studying asymptotically flat manifolds is weighted function spaces. Let $(N, g)$ be an asymptotically flat manifold, with asymptotic coordinates $\left\{z^{i}\right\}$ on $N_{\infty}$. Let $\rho(z)=|z|$ on $N_{\infty}$, extended to a smooth positive function on all of $N$. For $q \geqslant 1$ and $\beta \in \mathbf{R}$ we define the weighted Lebesgue space $L_{0, \beta}^{q}(N)$ as the set of locally integrable functions $u$ for which the norm

$$
\|u\|_{q, 0, \beta}=\left(\int_{N}\left|\rho^{-\beta} u\right|^{q} \rho^{-n} d V_{g}\right)^{1 / q}
$$


is finite. For a nonnegative integer $k$ the weighted Sobolev space $L_{k, \beta}^{q}(N)$ is the set of $u$ for which $\left|\nabla^{i} u\right| \in L_{0, \beta-i}^{q}(N)$ for $0 \leqslant i \leqslant k$, with norm

$$
\|u\|_{q, k, \beta}=\sum_{i=0}^{k}\left\|\nabla^{i} u\right\|_{q, 0, \beta-i}
$$

We note that $C_{c}^{\infty}(N)$ is dense in $L_{k, \beta}^{p}(N)$.

We also define the weighted $C^{k}$ space $C_{\beta}^{k}(N)$ as the set of $C^{k}$ functions $u$ for which the norm

$$
\|u\|_{C_{\beta}^{k}}=\sum_{i=0}^{k} \sup _{N} \rho^{-\beta+i}\left|\nabla^{i} u\right|
$$

is finite. The weighted Hölder space $C_{\beta}^{k, \alpha}(N)$ is defined for $0<\alpha<1$ as the set of $u \in C_{\beta}^{k}(N)$ for which the norm

$$
\|u\|_{C_{\beta}^{k, \alpha}}=\|u\|_{C_{\beta}^{k}}+\sup _{x, y}(\min \rho(x), \rho(y))^{-\beta+k+\alpha} \frac{\left|\nabla^{k} u(x)-\nabla^{k} u(y)\right|}{|x-y|^{\alpha}}
$$

is finite. (Here, as in $\S 2$, the supremum is over all $x \neq y$ such that $y$ is contained in a normal coordinate neighborhood of $x$, and $\nabla^{k} u(y)$ is the tensor at $x$ obtained by parallel transport along the radial geodesic from $x$ to $y$.) It is easy to check that multiplication is a continuous map from $C_{\beta}^{0, \alpha} \times C_{\delta}^{0, \alpha}$ to $C_{\beta+\delta}^{0, \alpha}$.

Note that the definitions of the weighted spaces depend on the distance function' $\rho$, and thereby on a choice of asymptotic coordinates. However, it is easy to see that $\rho$ is uniformly equivalent to the geodesic distance from an arbitrary fixed point in $N$ as $\rho \rightarrow \infty$, so all choices of $\rho$ define equivalent norms.

The theory of elliptic operators on these spaces was introduced by Nirenberg and Walker [NW], and has been developed extensively by Lockhart [Lo], McOwen [M], Cantor [C], Bartnik [B], Choquet-Bruhat and Christodoulou [CBC], Chaljub-Simon and Choquet-Bruhat [CSCB], and others.

We remark that the definition of asymptotically flat manifolds can be extended to manifolds with two or more asymptotic 'ends', and all of the results of this section extend easily to that case (see [C], for example).

In the literature there are several ways of indexing weighted spaces. Following Bartnik, we have chosen our definitions so that the index $\beta$ reflects order of growth: functions in $C_{\beta}^{k, \alpha}$ or $L_{k, \beta}^{q}$ grow at most like $\rho^{\beta}$. Indeed, it follows immediately from the definitions that $f \in C_{\beta}^{k, \alpha}$ implies $f=O\left(\rho^{\beta}\right)$. The corresponding fact for $L_{k, \beta}^{q}$ is a consequence of the following Sobolev lemma for weighted spaces.

Lemma 9.1 (Weighted Sobolev lemma). Suppose $q>1$ and $l-k-\alpha>$ $n / q$. For each $\varepsilon>0$ there are continuous embeddings $C_{\beta-\varepsilon}^{l, \alpha} \subset L_{l, \beta}^{q} \subset C_{\beta}^{k, \alpha}$. In particular, if $f \in L_{l, \beta}^{q}$ with $l>n / q$ then $f=O\left(\rho^{\beta}\right)$.

Proof. The first embedding is easily obtained from the definitions. The second is proved for weighted $C^{k}$ spaces on $\mathbf{R}^{n}$ by Cantor [C]. Bartnik sketches a proof of the general case in $[\mathbf{B}]$; although his definition of the weighted Hölder spaces differs slightly from ours, the same method applies. 
The beauty of the weighted spaces is that they give us analogues of many of the global elliptic regularity results that we stated for compact manifolds in $\$ 2$. In general, these theorems are not true on noncompact manifolds without weights.

THEOREM 9.2 (WeIGHTED ELLIPTIC REgUlaRITY). Let $(N, g)$ be asymptotically flat with $g_{i j}-\delta_{i j} \in C_{-\tau}^{1, \alpha}(N), \tau>0$, and $q>1$.

(a) For all $u \in L_{2, \beta}^{q}(N)$,

$$
\|u\|_{q, 2, \beta} \leqslant C\left(\|\Delta u\|_{q, 0, \beta-2}+\|u\|_{q, 0, \beta}\right) .
$$

(b) $\Delta: \quad L_{2, \beta}^{q}(N) \rightarrow L_{0, \beta-2}^{q}(N)$ is an isomorphism iff $2-n<\beta<0$. In addition, if $\beta \notin \mathbf{Z}, \Delta$ is surjective for all $\beta>2-n$ and injective for all $\beta<0$, and the dimension of $\operatorname{ker} \Delta$ is the same as that of the kernel of the Euclidean Laplacian $\Delta_{0}: L_{2, \beta}^{q}\left(\mathbf{R}^{n}\right) \rightarrow L_{0, \beta-2}^{q}\left(\mathbf{R}^{n}\right)$, which is the space of harmonic polynomials of degree $<\beta$.

(c) If $u \in C_{\beta}^{0}(N)$ and $\Delta u \in C_{\beta-2}^{0, \alpha}(N)$, then $u \in C_{\beta}^{2, \alpha}(N)$ and

$$
\|u\|_{C_{\beta}^{2, \alpha}} \leqslant C\left(\|\Delta u\|_{C_{\beta-2}^{0, \alpha}}+\|u\|_{C_{\beta}^{0}}\right) \text {. }
$$

(d) If $2-n<\beta<0, h \in C_{\delta}^{0, \alpha}(N)$ for some $\delta<-2$, and in addition the operator $\Delta+h: C_{\beta}^{2, \alpha}(N) \rightarrow C_{\beta-2}^{0, \alpha}(N)$ is injective, then it is an isomorphism.

Proof. Part (a) is essentially Proposition 1.6 in [B]. Part (b) is proved in [C, $\mathbf{M}, \mathbf{L o}$, and B]. The estimate in part (c) is proved in [CSCB] in the case $n=3$ and $2-n<\beta<0$, and under the assumption that $u \in C_{\beta}^{2, \alpha}(N)$; in the general case stated here, the fact that $u \in C_{\text {loc }}^{2, \alpha}(N)$ follows from local elliptic regularity, and the global estimate is proved by the same techniques as in [CSCB]. Finally, (d) is proved in [CSCB].

As an immediate application of these results, we prove the existence of a preferred system of coordinates near infinity, called harmonic coordinates (cf. [B]).

TheOREM 9.3 (HARMONIC COORDINATES). Suppose $(N, g)$ is asymptotically flat with $g_{i j}-\delta_{i j} \in C_{-\tau}^{1, \alpha}(N), \tau>(n-2) / 2$, and let $\left\{z^{i}\right\}$ be any asymptotic coordinates on $N_{\infty}$. There exist $C^{\infty}$ functions $\left\{y^{1}, \ldots, y^{n}\right\}$ on $N$ satisfying $\Delta y^{i}=0$ and

$$
\begin{array}{ll}
z^{i}-y^{i} \in C_{-\tau+1}^{2, \alpha}\left(N_{\infty}\right) & \text { if } n \geqslant 4, \\
z^{i}-y^{i} \in C_{-\tau+1+\varepsilon}^{2, \alpha}\left(N_{\infty}\right) & \text { if } n=3,
\end{array}
$$

such that $\left\{y^{i}\right\}$ form asymptotic coordinates for $N$ on some set $\left\{\rho \geqslant R_{0}\right\}$.

Proof. Extend the functions $z^{i}$ arbitrarily to smooth functions on $N$. Then the fact that $\tau>(n-2) / 2$ implies that

$$
\Delta z^{i}=-g^{j k}\left(z^{i}\right)_{, j k}=g^{j k} \Gamma_{j k}^{i} \in C_{-\tau-1}^{0, \alpha}(N) .
$$

If $n \geqslant 4$, Theorem 9.2(d) shows there exist functions $u^{i} \in C_{-\tau+1}^{2, \alpha}(N)$ such that $\Delta u^{i}=\Delta z^{i}$. If $n=3$, however, the index $\beta=-\tau+1$ may be positive, so this approach fails. Instead, note that $\Delta z^{i} \in L_{0,-\tau-1+\varepsilon}^{q}(N)$ for any $q>1, \varepsilon>0$ by Lemma 9.1. If we set $\beta=-\tau+1+\varepsilon$, and assume $\varepsilon$ is chosen so that $\beta \notin \mathbf{Z}$ 
and $\beta>2-n$, then Theorem 9.2(b) shows there exists $u^{i} \in L_{2, \beta}^{q}(N)$ with $\Delta u^{i}=\Delta z^{i}$. If $q>n$ the weighted Sobolev lemma implies $u^{i} \in C_{\beta}^{0, \alpha}(N)$, and thus Theorem 9.2(c) shows that $u^{i} \in C_{-\tau+1+\varepsilon}^{2, \alpha}(N)$.

Set $y^{i}=z^{i}-u^{i}$. The fact that $\left|\nabla u^{i}\right|=O\left(\rho^{-\tau+\varepsilon}\right)$ means that the differentials of the functions $y^{i}$ are independent near infinity; a simple application of the mean value theorem then shows that the resulting map $y: M \rightarrow \mathbf{R}^{n}$ is injective for $|y|$ large enough, and thus $\left\{y^{i}\right\}$ form coordinates near infinity. The formula for the metric coefficients in $y$-coordinates shows that $\left\{y^{i}\right\}$ are indeed asymptotic coordinates.

The weighted Hölder spaces provide us with an appropriate topology in which to study the mass functional introduced in $\S 8$. It turns out, as we will see in the next lemma, that finiteness of the mass is intimately related to integrability of the scalar curvature. For $\tau>(n-2) / 2$ we define $\mathscr{M}_{\tau}$ to be the set of all $C^{\infty}$ metrics on $N$ such that (in some asymptotic coordinates)

$$
g_{i j}-\delta_{i j} \in C_{-\tau}^{1, \alpha}\left(N_{\infty}\right), \quad S_{g} \in L^{1}(N) .
$$

In $z$-coordinates on $N_{\infty}$,

$$
\begin{aligned}
S & =g^{j k}\left(\partial_{i} \Gamma_{j k}^{i}-\partial_{k} \Gamma_{i j}^{i}+\Gamma_{i l}^{i} \Gamma_{j k}^{l}-\Gamma_{k l}^{i} \Gamma_{i j}^{l}\right) \\
& =\partial_{j}\left(\partial_{i} g_{i j}-\partial_{j} g_{i i}\right)+O\left(\rho^{-2 \tau-2}\right) .
\end{aligned}
$$

Choosing a base metric $g_{0} \in \mathscr{M}_{\tau}$, it follows that the scalar curvature of a metric $g_{0}+b$ is in $L^{1}$ iff $\partial_{j} \partial_{i} b_{i j}-\partial_{j} \partial_{j} b_{i i} \in L^{1}\left(N_{\infty}\right)$. Thus we can identify $\mathscr{M}_{\tau}$ with a subset of the affine space

$$
\left\{g_{0}+b: b \in C_{-\tau}^{1, \alpha}(N) \text { and } \partial_{j} \partial_{i} b_{i j}-\partial_{j} \partial_{j} b_{i i} \in L^{1}\left(N_{\infty}\right)\right\}
$$

with the obvious norm. In this topology $g_{k} \rightarrow g$ in $\mathscr{M}_{\tau}$ if $\left\|g_{k}-g\right\|_{C_{-\tau}^{1, \alpha}} \rightarrow 0$ and $\left\|S_{k}-S\right\|_{1} \rightarrow 0$.

LEMMA 9.4. If $\tau>(n-2) / 2$, the mass functional is a continuous affine functional on $\mathscr{M}_{\tau}$, and thus is infinitely differentiable.

Proof. The mass is evidently an affine function from its definition. From (9.2), $S=-\nabla^{*} \mu+O\left(\rho^{-2 \tau-2}\right)$ on $N_{\infty}$, where $\mu$ is the mass-density field as in Definition 8.2. Let $\eta$ be a smooth cutoff function which is supported in $N_{\infty}$ and identically one for large $\rho$. Then by the divergence theorem,

$$
\begin{aligned}
m(g) & \left.=\lim _{R \rightarrow \infty} \int_{S_{R}} \mu\right\lrcorner d z=\lim _{r \rightarrow \infty} \int_{S_{R}} \eta\langle\mu, N\rangle d \omega_{R} \\
& =\int_{N}\left(-\eta \nabla^{*} \mu+\langle\mu, \nabla \eta\rangle\right) d V_{g} .
\end{aligned}
$$

Now $g \in \mathscr{M}_{\tau}$ implies $\eta \nabla^{*} \mu \in L^{1}(N)$ and $\langle\mu, \nabla \eta\rangle \in C_{c}^{0, \alpha}(M) \subset L^{1}(N)$. Thus the last expression above is continuous on $\mathscr{M}_{\tau}$.

We remark that the above proof can be adapted to show that the $C_{-\tau}^{1, \alpha}$ norm can be replaced by the $L_{1,(2-n) / 2}^{q}$ norm on $\mathscr{M}_{\tau}$. 
We can now show that asymptotic coordinates are unique up to an obvious equivalence relation. Suppose $\left\{z^{i}\right\}$ are asymptotic coordinates with respect to which

$$
g_{i j}-\delta_{i j} \in C_{-\tau}^{1, \alpha}\left(N_{\infty}\right),
$$

and $\left\{\tilde{z}^{i}\right\}$ are any other coordinates on $N_{\infty}$ such that

$$
\tilde{z}(z)-E(z) \in C_{-\tau+1}^{2, \alpha}\left(N_{\infty}\right)
$$

for some Euclidean motion $E: \mathbf{R}^{n} \rightarrow \mathbf{R}^{n}$. Then it is clear from the definitions that (9.3) holds also in $\tilde{z}$-coordinates. It is an important fact that all choices of asymptotic coordinates are related in this way if $N$ is asymptotically flat of sufficiently high order. The proof (in the three-dimensional case) is due to Robert Bartnik.

THEOREM 9.5 (BARTNIK [B]). Suppose $(N, g)$ is asymptotically flat, and $\left\{z^{i}\right\}$, $\left\{\tilde{z}^{i}\right\}$ are any two choices of asymptotic coordinates with respect to which (9.3) holds with $\tau>(n-2) / 2$. Then there is a Euclidean motion $E$ such that (9.4) holds ( possibly with $\tau$ replaced by $\tau-\varepsilon$ for some small $\varepsilon>0$ ).

Proof. Let $\left\{y^{i}\right\}$ and $\left\{\tilde{y}^{i}\right\}$ be harmonic coordinates asymptotic to $\left\{z^{i}\right\}$ and $\left\{\tilde{z}^{i}\right\}$ respectively, obtained from Theorem 9.3. Observe that $y^{i}, \tilde{y}^{i} \in L_{2, \eta}^{q}(N)$ for any $1<\eta<2$. By Theorem 9.2(b), the dimension of ker $\Delta$ on $L_{2, \eta}^{q}(N)$ is the same as that of the Euclidean Laplacian on $L_{2, \eta}^{q}\left(\mathbf{R}^{n}\right)$, which is spanned by the linear functions and the constants. Thus $\left\{y^{1}, \ldots, y^{n}, 1\right\}$ form a basis for $\operatorname{ker} \Delta \subset L_{2, \eta}^{q}(N)$, and so there exist a matrix $A_{j}^{i}$ and constants $b^{i}$ so that

$$
\tilde{y}^{i}=A_{j}^{i} y^{j}+b^{i} .
$$

The fact that the metric $g$ is asymptotically flat with respect to both $\left\{y^{i}\right\}$ and $\left\{\tilde{y}^{i}\right\}$ shows that $A_{j}^{i}$ must be orthogonal. Combining (9.1) and (9.5) now yields the result.

The definition of mass involves a choice of asymptotic coordinates. But it follows from Bartnik's result that the mass is in fact an invariant of the metric.

THEOREM 9.6 [ADM, B]. If $N$ is asymptotically flat with metric $g \in \mathscr{M}_{\tau}$, $\tau>(n-2) / 2$, then $m(g)$ depends only on the metric $g$.

Proof. Suppose $\left\{z^{i}\right\}$ and $\left\{\tilde{z}^{i}\right\}$ are two choices of asymptotic coordinates on $N_{\infty}$. By Theorem 9.5, after composing with a Euclidean motion (and replacing $\tau$ by $\tau-\varepsilon$ if necessary) we may assume that

$$
\tilde{z}^{i}=z^{i}+\varphi^{i}, \quad \text { where } \varphi^{i} \in C_{-\tau+1}^{2, \alpha}\left(N_{\infty}\right) .
$$

The first key observation is that the radial distance functions $\rho=|z|$ and $\tilde{\rho}=|\tilde{z}|$ are related by $C^{-1} \tilde{\rho} \leqslant \rho \leqslant C \tilde{\rho}$ for some positive constant $C$. Thus if $S_{R}$ and $\tilde{S}_{R}$ represent the spheres $\{\rho=R\}$ and $\{\tilde{\rho}=R\}$ respectively, and $A_{R}$ is the annulus $\left\{C^{-1} R \leqslant \rho \leqslant C R\right\}$, the divergence theorem shows

$$
\left.\left.\mid \int_{\tilde{S}_{R}} \mu\right\lrcorner d z-\int_{S_{R}} \mu\right\lrcorner d z\left|\leqslant \int_{A_{R}}\right| \nabla * \mu \mid d z .
$$

This last integral goes to zero as $R \rightarrow \infty$, since $\nabla^{*} \mu \in L^{1}$ by (9.2). Therefore we can replace $S_{R}$ by $\tilde{S}_{R}$ in the definition of $m(g)$ without changing the mass. 
If $g_{i j}$ and $\tilde{g}_{i j}$ are the components of the metric in the two coordinate systems, then, writing $\partial_{i}=\partial / \partial z^{i}$ and $\tilde{\partial}_{k}=\partial / \partial \tilde{z}^{k}=\partial_{k}-\partial_{k} \varphi^{l} \partial_{l}+O\left(\rho^{-\tau}\right)$,

$$
\begin{gathered}
\tilde{g}_{i j}=g_{i j}-\partial_{i} \varphi^{j}-\partial_{j} \varphi^{i}+O\left(\rho^{-2 \tau}\right), \\
\tilde{\partial}_{k} \tilde{g}_{i j}=\partial_{k} g_{i j}-\partial_{k} \partial_{i} \varphi^{j}-\partial_{k} \partial_{j} \varphi^{i}+O\left(\rho^{-2 \tau-1}\right) .
\end{gathered}
$$

Therefore the mass density field $\mu=\left(\partial_{i} g_{i j}-\partial_{j} g_{i i}\right) \partial j$ is

$$
\mu=\tilde{\mu}+\left(\partial_{i} \partial_{i} \varphi^{j}-\partial_{i} \partial_{j} \varphi^{i}\right) \partial_{j}+O\left(\rho^{-2 \tau-1}\right) .
$$

Since the volume forms of $\left\{z^{i}\right\}$ and $\left\{\tilde{z}^{i}\right\}$ are related by $d z=d \tilde{z}+O\left(\rho^{-\tau}\right)$,

$$
\mu\lrcorner d z=\tilde{\mu}\lrcorner d \tilde{z}+d \eta+O\left(\rho^{-2 \tau-1}\right),
$$

where $\eta$ is the $(n-2)$-form

$$
\left.\left.\eta=\partial_{i} \varphi^{j}\left(\partial_{i}\right\lrcorner \partial_{j}\right\lrcorner d z\right) .
$$

Integrating this over $\tilde{S}_{R}$ and taking the limit, we conclude that $m(g)=m(\tilde{g})$.

For the Yamabe problem, we are interested in the asymptotically flat manifold $\hat{M}$ obtained by stereographic projection from a compact manifold $M$. The simplest case is when $M$ is locally conformally flat, for then

$$
\hat{g}_{i j}(z)=\gamma(z)^{p-2} \delta_{i j},
$$

where

$$
\gamma(z)=1+A \rho^{2-n}+O^{\prime \prime}\left(\rho^{1-n}\right) .
$$

Using (7.2) and (7.3), one can calculate easily that the constant $A$ is related to the mass and the distortion coefficient by $2(n-1) A=\mu=\frac{1}{2} m(\hat{g})$. More generally, we have the following relation between $\mu$ and $m(\hat{g})$.

LEMMA 9.7. Let $\hat{M}$ be the stereographic projection of $M$ from $P \in M$, and $\mu$ the distortion coefficient computed with respect to inverted conformal normal coordinates. If $n<6$ or $M$ is conformally flat in a neighborhood of $P$, then $\mu$ $=\frac{1}{2} m(\hat{g})$.

Proof. Theorem 6.5 and the fact that the scalar curvature of $\hat{g}$ is identically zero show that in these cases $\hat{g} \in \mathscr{M}_{\tau}$ with $\tau>(n-2) / 2$, so the mass is defined. It can be expressed in terms of radial derivatives as follows. First note that on the sphere $\left.\left.S_{\rho}, \partial_{j}\right\lrcorner d z=\rho^{-1} z^{j} d \omega_{\rho}=\rho^{-2} z^{j} z^{k} \partial_{k}\right\lrcorner d z$, so the mass formula becomes

$$
\left.m(g)=\lim _{\rho \rightarrow \infty} \omega^{-1} \int_{S_{\rho}}\left(\rho^{-2} z^{j} z^{k} \partial_{i} \hat{g}_{i j}-\partial_{k} \hat{g}_{i i}\right) \partial_{k}\right\lrcorner d z .
$$

Now observe that $\hat{g}_{\rho \rho}=\hat{g}\left(\partial_{\rho}, \partial_{\rho}\right)=\rho^{-2} z^{k} z^{j} \hat{g}_{k j}, \partial_{\rho} \hat{g}_{\rho \rho}=\rho^{-3} z^{i} z^{j} z^{k} \partial_{i} \hat{g}_{k j}$, and that the $(n-2)$-form $\left.\left.\eta=z^{j} z^{k} \hat{g}_{i j} \partial_{i}\right\lrcorner \partial_{k}\right\lrcorner d z$ satisfies

$$
\left.d \eta=\left(z^{j} z^{k} \partial_{i} \hat{g}_{i j}-z^{j} z^{i} \partial_{i} \hat{g}_{k j}+z^{k} \hat{g}_{i i}-n z^{j} \hat{g}_{k j}\right) \partial_{k}\right\lrcorner d z .
$$

Hence

$$
m(g)=\lim _{\rho \rightarrow \infty} \omega^{-1} \int_{S_{\rho}} \partial_{\rho}\left(\hat{g}_{\rho \rho}-\hat{g}_{i i}\right)+\rho^{-1}\left(n \hat{g}_{\rho \rho}-\hat{g}_{i i}\right) d \omega_{\rho}
$$


This simplifies greatly in inverted conformal normal coordinates, where we have $\hat{g}_{\rho \rho}=\gamma^{p-2}$ and det $\hat{g}=\gamma^{2 p}=1+O\left(\rho^{2-n}\right)$. Thus

$$
\begin{aligned}
n \partial_{\rho} \hat{g}_{\rho \rho} & =n(p-2) \gamma^{p-3} \partial_{\rho} \gamma=\gamma^{p-2} \partial_{\rho}(\log \operatorname{det} \hat{g}) \\
& =\partial_{\rho} \hat{g}_{i i}+O\left(\rho^{-2 \tau-1}\right) .
\end{aligned}
$$

Integrating along rays from infinity and noting that $n \hat{\mathrm{g}}_{\rho \rho}=n=\hat{\mathrm{g}}_{i i}$ at infinity shows that $n \hat{g}_{\rho \rho}=\hat{g}_{i i}+O\left(\rho^{-2 \tau}\right)$. Then (9.6) becomes

$$
m(\hat{g})=\lim _{\rho \rightarrow \infty} \omega^{-1} \int_{S_{\rho}} a \partial_{\rho} \gamma d \omega_{\rho}
$$

so $m(\hat{g})=2 \mu$ by (7.4).

10. The positive mass theorem. We will now sketch the proof of the general positive mass theorem, relying on the work of Schoen and Yau. The discussion of $\S 9$ indicates that the natural setting for studying the mass is an asymptotically flat manifold with metric $g \in \mathscr{M}_{\tau}$ for some $\tau>(n-2) / 2$. A general version of the theorem is the following.

THEOREM 10.1 (Positive MASS THEOREM). Let $(N, g)$ be an asymptotically flat Riemannian manifold of dimension $n \geqslant 3$ with metric $g \in \mathscr{M}_{\tau}, \tau>$ $(n-2) / 2$, and nonnegative scalar curvature. Then its mass $m(g)$ is nonnegative, with $m(g)=0$ if and only if $(N, g)$ is isometric to $\mathbf{R}^{n}$ with its Euclidean metric.

Schoen and Yau [S] have recently announced a proof of this theorem in the case $\tau=n-2$. We begin our sketch of the proof with a weaker but much simpler theorem, which shows that the mass is nonnegative provided the Ricci curvature is nonnegative. This argument extends earlier results of E. Witten, $R$. Bartnik, and R. Schoen. It is a noncompact version of Bochner's vanishing theorem.

Proposition 10.2. Suppose $(N, g)$ is asymptotically flat with $g \in \mathscr{M}_{\tau}$ for some $\tau>(n-2) / 2$, and the Ricci tensor of $g$ is nonnegative. Then the mass $m(g)$ is nonnegative, with $m(g)=0$ if and only if $(N, g)$ is isometric to $\mathbf{R}^{n}$ with its Euclidean metric.

Proof. By Theorem 9.6, we may use any convenient coordinates to compute the mass. Let $\left\{y^{i}\right\}$ be harmonic coordinates as in Theorem 9.3, so $\Delta y^{i}=$ $d^{*} d y^{i}=0$. Then the 1 -forms $\omega^{i}=d y^{i}$ satisfy $d \omega^{i}=d^{*} \omega^{i}=0$. From the Ricci identity (2.2) we obtain the classical Bochner formula for a 1-form $\omega$ :

$$
\Delta \omega=\left(d d^{*}+d^{*} d\right) \omega=\left(\nabla^{*} \nabla+\mathrm{Ric}\right) \omega .
$$

Applying this to $\omega^{i}$, taking inner products, summing over $i$, and integrating by parts over $N_{R}=\{\rho \leqslant R\}$ yields

$$
\left.\sum_{i} \int_{N_{R}}\left|\nabla \omega^{i}\right|^{2}+\operatorname{Ric}\left(\omega^{i}, \omega^{i}\right) d V_{g}=\sum_{i, j} \int_{S_{R}} g^{j k}\left\langle\omega^{i}, \nabla_{j} \omega^{i}\right\rangle \partial_{k}\right\lrcorner d V_{g}
$$


But observe that $\nabla_{j} \omega^{i}=\nabla_{j} d y^{i}=-\Gamma_{j k}^{i} d y^{k}$, and in harmonic coordinates $0=\Delta y^{i}=g^{j k} \Gamma_{j k}^{i}$. Therefore the mass-density field on $N_{\infty}$ is

$$
\begin{aligned}
\mu & =\left(\Gamma_{i i}^{j}-\Gamma_{i j}^{i}\right) \partial_{j}+O\left(\rho^{-2 \tau-1}\right) \\
& =\sum_{i, j}\left\langle\omega^{i}, \nabla_{j} \omega^{i}\right\rangle \partial_{j}+O\left(\rho^{-2 \tau-1}\right) .
\end{aligned}
$$

Integrating over $S_{R}$ and taking the limit as $R \rightarrow \infty$ yields

$$
m(g)=\omega^{-1} \sum_{i} \int_{N}\left(\left|\nabla \omega^{i}\right|^{2}+\operatorname{Ric}\left(\omega^{i}, \omega^{i}\right)\right) d V_{g} \geqslant 0 .
$$

Now suppose the mass is zero. Then the above formula shows that $\nabla \omega^{i}=0$ for each $i$. Thus $\nabla\left\langle\omega^{i}, \omega^{j}\right\rangle=\left\langle\nabla \omega^{i}, \omega^{j}\right\rangle+\left\langle\omega^{i}, \nabla \omega^{j}\right\rangle \equiv 0$, and since the coframe $\left\{\omega^{i}\right\}$ is orthonormal at infinity it is orthonormal everywhere. This implies that the map $y: N \rightarrow \mathbf{R}^{n}$ is a local isometry. In fact, since it is a proper local diffeomorphism, it is a covering map, and therefore a global isometry. Thus $N$ is isometric to Euclidean $\mathbf{R}^{n}$.

In 1981, Witten discovered that a variant of this argument, using spinors in place of the one-forms $\omega^{i}$, gives a simple proof of the positive mass theorem for a manifold with a spin structure.

THEOREM 10.3 (WITTEN [W]). Suppose $N$ is an asymptotically flat spin manifold of dimension $n \geqslant 3$, with metric $g=\delta_{i j}+h_{i j}$, where $h_{i j} \in C_{-\tau}^{2, \alpha}(N)$ for some $\tau>(n-2) / 2$. If $N$ has finite mass and nonnegative scalar curvature, then $m(g) \geqslant 0$.

Since the proof is quite simple, we include it in the appendix. ${ }^{1}$

We remark that the proof extends easily to spin manifolds with several asymptotic ends, as mentioned in $\$ 9$. In that case there is a mass associated with each end, and the theorem states that each mass is nonnegative (see [PT]).

The definition of a spin manifold implies orientability. But the above remark allows us to extend Witten's theorem to certain nonorientable manifolds: if $N$ is an asymptotically flat manifold whose orientable double cover $\tilde{N}$ is spin, the theorem applied to $\tilde{N}$ (which has two ends) implies that the mass of $N$ is nonnegative.

Another special case - that of locally conformally flat manifolds-is directly applicable to the Yamabe problem. A new proof for this case has recently been given by Schoen and Yau [SY6]. Their theorem is as follows:

THEOREM 10.4. Suppose $(\hat{M}, \hat{g})$ is a stereographic projection of a compact, locally conformally flat manifold $M$ of dimension $n \geqslant 4$ with $\lambda(M)>0$. Then $m(\hat{g})>0$ unless $\hat{M}$ is isometric to $\mathbf{R}^{n}$.

The proof of this theorem is based on a careful analysis of the developing map, a conformal map from the universal cover of $M$ to the sphere. Schoen and Yau showed that the hypotheses on $M$ imply that the developing map is

\footnotetext{
${ }^{1}$ At the time this was first written, no proof of this version of Witten's theorem had appeared in the literature. Since then, Bartnik has independently written a proof and included it in the published version of [B].
} 
injective; the result then follows by comparing the Green function on $M$ with that on the sphere.

The proof of the general positive mass theorem will be carried out in three steps. The heart of the proof is the following lemma, which shows that $m(g) \geqslant 0$ if $g$ has a particularly simple asymptotic form.

Lemma 10.5 (Schoen ANd YAu [SY1, SY2, SY4, SY5]). Let $(N, g)$ be an asymptotically flat manifold with metric $g$ of the following special form:

$$
g_{i j}(z)=\left(1+A \rho^{2-n}\right) \delta_{i j}+\Phi_{i j}(z),
$$

where $A$ is a constant and $\Phi_{i j} \in C_{1-n}^{5}\left(N_{\infty}\right)$. If the scalar curvature of $g$ is nonnegative, then $m(g)=(n-2)(n-1) A \geqslant 0$.

Proof. This is proved by induction on $n$. The case $n=3$ is a consequence of Witten's theorem (and the remark following it) since every orientable 3-manifold admits a spin structure. (It also follows from Schoen and Yau's original proof of the positive mass theorem [SY1].)

We give only a brief sketch of the inductive step. One begins by assuming the mass is negative. This assumption can be used to prove the existence of an embedded asymptotically flat minimal hypersurface $H \subset N$. By conformally changing the induced metric on $H$ to a metric $\tilde{g}$ of zero scalar curvature, and analyzing the second variation of the minimal surface equation, one concludes that $\tilde{g}$ has negative mass, contradicting the inductive hypothesis.

This argument is given in the case of dimension 4 in [SY2]. The general result was announced in [S], but a proof has not yet appeared. If $n \leqslant 7$ the argument proceeds just as in the 4-dimensional case. For $n \geqslant 7$, the minimal hypersurface may have singularities, and one must analyze these carefully, in effect proving the theorem for manifolds with certain types of singularities.

The next step is to remove the restrictive assumptions on the metric $g$.

LEMMA 10.6. Under the hypotheses of Theorem 10.1, $m(g) \geqslant 0$.

Proof. We will reduce the theorem to the restricted case above by "flattening" $g \in \mathscr{M}_{\tau}$ on the asymptotic end $N_{\infty}$, and then conformally changing back to nonnegative scalar curvature.

Let $\left\{z^{i}\right\}$ be asymptotic coordinates on $N_{\infty}$, and let $\rho: N \rightarrow \mathbf{R}$ be the radial distance function as in $\$ 9$. Fix a smooth cutoff function $\eta$ on $\mathbf{R}$ with $0 \leqslant \eta \leqslant 1, \eta(t)=1$ for $t \leqslant 1$, and $\eta(t)=0$ for $t \geqslant 2$. For $R>0$ define $\eta_{R}$ : $N \rightarrow \mathbf{R}$ by $\eta_{R}(z)=\eta\left(R^{-1} \rho(z)\right)$. Write $g_{i j}=\delta_{i j}+b_{i j}$ with $b_{i j} \in C_{-\tau}^{1, \alpha}(N)$, and consider the flattened metrics

$$
\left(g_{R}\right)_{i j}=\delta_{i j}+\eta_{R} b_{i j} .
$$

These metrics may have negative scalar curvature at some points. A natural way to restore positive scalar curvature is to seek a metric $\tilde{g}_{R}$ conformal to $g_{R}$ which has prescribed nonnegative scalar curvature, say, equal to $\eta_{R} S$. This would require solving the nonlinear prescribed scalar curvature equation $\square_{R} \varphi=\eta_{R} S \varphi^{p-1}$. A much simpler approach is to solve the linear equation

$$
\square_{R} \varphi_{R}=\eta_{R} S \varphi_{R}
$$


for $\varphi_{R}>0$, for then the transformation law (1.1) shows that the metric $\tilde{g}_{R}=\varphi_{R}^{p-2} g_{R}$ has scalar curvature

$$
\tilde{S}_{R}=\varphi_{R}^{1-p} \square_{R} \varphi_{R}=\varphi_{R}^{2-p} \eta_{R} S \geqslant 0 .
$$

Writing $\varphi_{R}=1+\psi_{R}$ and $\gamma_{R}=S_{R}-\eta_{R} S$, (10.1) becomes

$$
\left(a \Delta_{R}+\gamma_{R}\right) \psi_{R}=-\gamma_{R} \text {. }
$$

To solve this we first note that, for any positive $\varepsilon, \gamma_{R} \rightarrow 0$ in $C_{-\tau-2+\varepsilon}^{0, \alpha}(N)$ as $R \rightarrow \infty$. Indeed, the usual coordinate formulas show that the Christoffel symbols of $g$ and $g_{R}=g+\left(\eta_{R}-1\right) b$ are related by $\Gamma_{R}-\eta_{R} \Gamma=O\left(b \cdot \partial \eta_{R}\right)$ and

$$
\gamma_{R}=S_{R}-\eta_{R} S=O\left(\partial \eta_{R} \cdot \partial b\right)+O\left(b \cdot \partial \partial \eta_{R}\right)+O\left(b \cdot\left|\partial \eta_{R}\right|^{2}\right)
$$

It follows from its definition that $\eta_{R}$ is bounded in $C_{0}^{k, \alpha}(N)$ for any $k$, and thus $\partial \eta_{R} \in C_{-1}^{0, \alpha}$ and $\partial \partial \eta_{R} \in C_{-2}^{0, \alpha}$, uniformly in $R$. Since multiplication is continuous from $C_{\beta}^{0, \alpha} \times C_{\delta}^{0, \alpha}$ to $C_{\beta+\delta}^{0, \alpha}$, the above formula shows that $\left\|\gamma_{R}\right\|_{C_{-\tau-2}^{0, \alpha}}$ is bounded independently of $R$. Since $\gamma_{R}$ is supported in the annulus $\{R \leqslant \rho$ $\leqslant 2 R\}$, this implies that $\left\|\gamma_{R}\right\|_{C_{-\tau-2+e}^{0, \alpha}} \rightarrow 0$ for any positive $\varepsilon$.

Since the Laplacian $\Delta$ of $g$ is injective on $L_{2,-\tau+2 \varepsilon}^{q}(N) \supset C_{-\tau+\varepsilon}^{2, \alpha}(N)$ by Theorem 9.2(b), part (d) of that theorem shows that $\Delta: C_{-\tau+\varepsilon}^{2, \alpha}(N) \rightarrow$ $C_{-\tau-2+\varepsilon}^{0, \alpha}(N)$ is an isomorphism. Thus the operator $a \Delta_{R}+\gamma_{R}$ will also have a bounded inverse if the operator norm of the perturbation term $a\left(\Delta_{R}-\Delta\right)+\gamma_{R}$ is small enough. The multiplicative property of weighted Hölder spaces shows that the operator norm of $\gamma_{R}$ is bounded by $\left\|\gamma_{R}\right\|_{C_{-2}^{0, \alpha}}$. On the other hand, one can calculate that

$$
\left\|\left(\Delta_{R}-\Delta\right) u\right\|_{C_{-\tau-2+\varepsilon}^{0, \alpha}} \leqslant\left\|g_{R}-g\right\|_{C_{0}^{1, \alpha}}\|u\|_{C_{-\tau+\varepsilon}^{2, \alpha},},
$$

and $g_{R} \rightarrow g$ in $C_{0}^{1, \alpha}(N)$. Thus $a \Delta_{R}+\gamma_{R}$ has a bounded inverse for large $R$, and we can solve (10.3) for $\psi_{R} \in C_{-\tau+\varepsilon}^{2, \alpha}(N)$ with $\left\|\psi_{R}\right\|_{C_{-\tau+\varepsilon}^{2, \alpha}} \rightarrow 0$. In particular, $\varphi_{R}=1+\psi_{R}$ is strictly positive for large $R$.

Now because the Laplacian $\Delta_{R}$ is equal to the Euclidean Laplacian in asymptotic coordinates for $\rho>2 R$, and $\gamma_{R}=0$ there, the functions $\psi_{R}$ are harmonic (in the Euclidean sense) near infinity. Consider the inversion $x \mapsto$ $|x|^{-2} x$ which sends a neighborhood $A$ of the origin in $\mathbf{R}^{n}$ to a neighborhood of infinity. It is easy to check that the function $\bar{\psi}_{R}(x)=|x|^{2-n} \psi_{R}\left(|x|^{-2} x\right)$ is harmonic on $A-\{0\}$. Since $\psi_{R}=O\left(\rho^{-\tau+\varepsilon}\right)$, we have $\bar{\psi}_{R}=O\left(|x|^{2-n+\tau-\varepsilon}\right)$, and so $\tau>(n-2) / 2$ implies $\bar{\psi}_{R} \in L^{p}(A)$. Thus the removable singularities result (Proposition 2.7) shows that $\bar{\psi}_{R}$ is weakly harmonic on $A$, and elliptic regularity shows that $\bar{\psi}_{R}$ is $C^{\infty}$. In particular, $\nabla^{k} \bar{\psi}_{R}(x)$ is bounded for all $k$. Inverting, we find that $\nabla^{k} \psi_{R}(z)=O\left(\rho^{2-n-k}\right)$ for all $k$.

Thus the metric $\tilde{g}_{R}=\varphi_{R}^{p-2} g_{R}$ satisfies the hypotheses of Lemma 10.5, so $m\left(\tilde{g}_{R}\right) \geqslant 0$.

The proof is completed by showing that $\tilde{g}_{R} \rightarrow g$ in the topology of $\mathscr{M}_{\tau-\varepsilon}$, which implies $m\left(\tilde{g}_{R}\right) \rightarrow m(g)$. Note that $g-g_{R}=\left(1-\eta_{R}\right) b$ is supported in $\{\rho \geqslant 2 R\}$ and bounded in $C_{-\tau}^{1, \alpha}(N)$ independently of $R$, and thus converges to zero in $C_{-\tau+\varepsilon}^{1, \alpha}(N)$. We have

$$
g-\tilde{g}_{R}=\left(g-g_{R}\right)+\left(1-\varphi_{R}^{p-2}\right) g_{R} .
$$


The first term goes to zero in $C_{-\tau+\varepsilon}^{1, \alpha}(N)$ by the previous remark, and the second goes to zero since $\varphi_{R} \rightarrow 1$ in that space. Finally,

$$
\left|S-\tilde{S}_{R}\right|=\left|S-\varphi_{R}^{2-p} \eta_{R} S\right| \leqslant 2\left(1-\eta_{R}\right)|S|
$$

since $\varphi_{R} \rightarrow 1$ uniformly, and thus $\int_{N}\left|S-\tilde{S}_{R}\right| \rightarrow 0$ since $S \in L^{1}(N)$.

The proof of the positive mass theorem is completed by examining the case $m(g)=0$.

LEMMA 10.7. If $(N, g)$ satisfies the hypotheses of Theorem 10.1 and has $m(g)=0$, then $N$ is isometric to $\mathbf{R}^{n}$ with its Euclidean metric.

Proof. We will use the variational formula (8.11) to show that the Ricci tensor of $g$ is zero. The lemma then follows from Proposition 10.2.

Let $h_{i j}$ be any smooth, compactly supported symmetric 2-tensor on $N$, and consider the family of metrics $g_{t}=g+t h$. These metrics may no longer have nonnegative scalar curvature. Following the method of Lemma 10.6 above, we change to the conformal metric $\tilde{g}_{t}=\varphi_{t}^{p-2} g_{t}$, where $\varphi_{t}$ is a positive solution to $\square_{t} \varphi_{t}=S \varphi_{t}$. This is equivalent to

$$
\left(a \Delta_{t}+\gamma_{t}\right) \psi_{t}=-\gamma_{t}
$$

where we have written $\varphi_{t}=1+\psi_{t}$ and $\gamma_{t}=S_{t}-S$. Since $\gamma_{t}$ is compactly supported and $S_{t} \rightarrow S$ in $C^{k}$ for any $k$ as $t \rightarrow 0$, it follows that $\left\|\gamma_{t}\right\|_{C_{-\tau-2}^{0 . \alpha}} \rightarrow 0$. Arguing as in the proof of Lemma 10.6, for small $t$ we can solve uniquely for

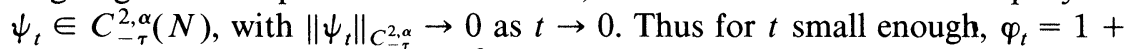
$\psi_{t}>0$, and the metric $\tilde{g}_{t}=\varphi_{t}^{p-2} g_{t}$ has scalar curvature

$$
\tilde{S}_{t}=\varphi_{t}^{1-p} \square_{t} \varphi_{t}=\varphi_{t}^{2-p} S \geqslant 0
$$

Now since $m(g)=0$, the previous lemma implies that $g$ is a minimum for the mass among the metrics $\tilde{g}_{t}$. Since $m$ is differentiable by Lemma 9.4, this means that $\dot{m}(g)$ is zero, where a dot denotes the derivative with respect to $t$ at $t=0$. We will apply the variational formula (8.11) to the family $\tilde{g}_{t}$. First note that $\varphi_{t}$ is differentiable with respect to $t$, since the family of operators $\left(a \Delta_{t}+\gamma_{t}\right)^{-1}$ depends differentiably on $t$. At $t=0$,

$$
\begin{aligned}
\frac{d}{d t} \tilde{g}_{t}= & (p-2) \dot{\varphi} g+h \\
\frac{d}{d t} d \tilde{V}_{t} & =\frac{d}{d t}\left(\operatorname{det} \tilde{g}_{t}\right)^{1 / 2} d x \\
& =\frac{1}{2}\left((p-2) \dot{\varphi} g_{j k}+h_{j k}\right) g^{j k} d V_{g}
\end{aligned}
$$

Thus, since $\tilde{S}_{t}=\varphi_{t}^{2-p} S$,

$$
\frac{d}{d t}\left(\tilde{S}_{t} d \tilde{V}_{t}\right)=(2-p) \dot{\varphi} S d V_{g}+\frac{1}{2} S\left((p-2) \dot{\varphi} g_{j k}+h_{j k}\right) g^{j k} d V_{g}
$$


From (8.11) we have

$$
\begin{aligned}
0=\dot{m}(g) & =\frac{d}{d t} \int_{N} \tilde{S}_{t} d \tilde{V}_{t}+\int_{N}\left\langle\left(\frac{d}{d t} \tilde{g}_{t}\right), G\right\rangle d V_{g} \\
& =\int_{N} h^{j k} R_{j k} d V_{g} .
\end{aligned}
$$

This holds for all compactly supported $h$, so $R_{j k} \equiv 0$ on $N$. This completes the proof of the general positive mass theorem.

11. Solution of the Yamabe problem. We now have all the ingredients needed to solve the Yamabe problem. The solution has proceeded in several steps. First, the analytic arguments of $\$ 4$ showed that we can obtain a solution provided that $\lambda(M)<\lambda\left(S^{n}\right)$. Second, the test function estimate of $\S 7$ reduced the problem to verifying the positivity of the distortion coefficient $\mu$ of the manifold $\hat{M}$ obtained by stereographic projection in conformal normal coordinates. In many cases this follows directly from the asymptotic expansions of Theorem 6.5. In the remaining cases it is a consequence of the positive mass theorem.

The YAMABE THEOREM. If $(M, g)$ is a compact Riemannian manifold of dimension $n \geqslant 3$, there exists a metric $\tilde{g}$ conformal to $g$ with constant scalar curvature $S=\lambda(M)$.

Proof. If $M$ is conformal to the standard sphere, then Theorem 3.2 shows that the problem is solved by the standard metric. For any other $M$, by Theorem 4.5 it suffices to show that $\lambda(M)<\lambda\left(S^{n}\right)$. If $\lambda(M) \leqslant 0$, this is obviously the case. If $\lambda(M)>0$, then by Theorem 7.2 it suffices to find a stereographic projection $\hat{M}$ of $M$ with positive distortion coefficient $\mu$.

If $n \geqslant 6$, we can calculate the expansion of the spherical density function $h$ directly from the expansion for $\gamma$ obtained in Theorem 6.5. Using formula (7.2) and the fact that

$$
\int_{S_{\rho}} z^{i} z^{j} d \omega_{\rho}=\frac{1}{n} \delta^{i j} \rho^{2} \omega_{\rho}
$$

we find that

$$
h(\rho)= \begin{cases}1+\frac{1}{96 n(n-6)}|W(P)|^{2} \rho^{-4}+O^{\prime \prime}\left(\rho^{-5}\right) & \text { if } n \geqslant 7 \\ 1+\frac{1}{576}|W(P)|^{2} \rho^{-4} \log \rho+O^{\prime \prime}\left(\rho^{-4}\right) & \text { if } n=6\end{cases}
$$

Thus if $M$ is not locally conformally flat we can choose a point $P \in M$ with $W(P) \neq 0$, and then $\mu=C|W(P)|^{2}>0$. On the other hand, if $n<6$ or $M$ is locally conformally flat, then Lemma 9.7 and the positive mass theorem show that $\mu>0$ unless $\hat{M}$ is conformal to $\mathbf{R}^{n}$, in which case $M$ is conformal to $S^{n}$. 
There are several questions related to the Yamabe problem that are of considerable current interest. Yau [Yau] and others have posed the Yamabe problem for complete noncompact Riemannian manifolds. Using the methods of weighted spaces discussed in $\S 9$, one can show that an asymptotically flat manifold $N$ of order $\tau>(n-2) / 2$ with positive Yamabe invariant $\lambda(N)$ has an asymptotically flat conformal metric of zero scalar curvature provided the scalar curvature satisfies $S \in C_{-\tau-2}^{0, \alpha}(N)$. More generally, Patricio Aviles and Robert McOwen [AM] have recently obtained some interesting results about conformal deformations of noncompact manifolds, including a proof that the Yamabe problem is solvable for complete Riemannian manifolds whose scalar curvature is negative near infinity. In [SY6], Schoen and Yau have made progress toward understanding which open submanifolds of the sphere admit complete conformal metrics of constant scalar curvature.

A second question concerns the properties of the functional $Q_{g}$. The solution of the Yamabe problem is based on showing that the minimum of $Q_{g}$ is realized. It would be interesting to have a better understanding of the analytic properties of $Q_{g}$, and in particular to examine the higher critical points. Recently Ding Weiyue [Wy] has shown that $Q_{\hat{g}}$ has infinitely many critical values on the sphere (although it follows from Obata's theorem that the minima are the only critical functions that are strictly positive). Schoen has shown that the convergence theorem 4.5 holds for sequences $\left\{\varphi_{s}\right\}$ that are solutions-but not necessarily minima- of the subcritical problem, and for which $\left\{Q_{g}\left(\varphi_{s}\right)\right\}$ is bounded.

In another direction, the Yamabe problem can also be posed for CR manifolds, the abstract models of real hypersurfaces in complex manifolds. A strictly pseudoconvex CR manifold is an odd-dimensional manifold with a given codimension 1 sub-bundle of its tangent bundle, which carries a complex structure and a hermitian metric (the Levi form) defined only up to a conformal factor. S. Webster [We] has defined a notion of covariant differentiation and curvature for the Levi form, and it is natural to ask whether the conformal factor can be chosen so that the Webster scalar curvature is constant. This is the $C R$ Yamabe problem.

The formal structure of this problem is identical in many respects to that of the Riemannian Yamabe problem. The analysis, however, is considerably more difficult since the operator that takes the place of the Laplacian is only subelliptic rather than elliptic. Nonetheless, by using more delicate Sobolev estimates, one can still prove the analogue of Theorem A; this has been done by Lee and David Jerison in [JL1 and JL2].

In order to determine when the problem can be solved, one must identify the extremals for the "model case", the sphere in $\mathbf{C}^{n+1}$. Using a generalization of Obata's argument, Lee and Jerison have shown in [JL3] that the CR Yamabe functional is minimized by the standard Levi form on the sphere and its images under CR automorphisms.

Once the extremals are known, one must calculate the asymptotic expansion for the Yamabe functional of an appropriate test function, and relate it to local $\mathrm{CR}$ invariants. This calculation is significantly more complicated than in the Riemannian case, and requires the construction of special coordinates analogous to conformal normal coordinates. In [JLA], Lee and Jerison have carried 
out this calculation and proved the following analogue of Theorem B: the CR Yamabe problem can be solved on CR manifolds of dimension $\geqslant 5$ that are not locally CR equivalent to the sphere ("locally spherical").

When the dimension is 3 or the manifold is locally spherical, this local approach does not work and we are in a situation analogous to Theorem C. In that case one needs an analogue of the positive mass theorem for CR manifolds. This problem is still completely open.

Appendix: Witten's proof. In this appendix we give a proof of Witten's Theorem 10.3, beginning with a rapid summary of the geometric context of spinors. More details on the constructions discussed here may be found in [PT].

The group $\mathrm{SO}(n), n \geqslant 3$, has a twofold universal covering group called $\operatorname{Spin}(n)$. This group has a fundamental unitary representation on a space $V$ of complex dimension $2^{n-2}$ called the spin representation $\rho: \operatorname{Spin}(n) \rightarrow \operatorname{Aut}(V)$. When $n$ is odd $V$ is irreducible, and when $n$ is even $V=V_{+} \oplus V_{-}$is the direct sum of two irreducible representations. In addition there is an equivariant linear Clifford multiplication map $c: \mathbf{R}^{n} \rightarrow \operatorname{End}(V)$. The map $c(x)$ is skewhermitian and satisfies $c(x) c(y)+c(y) c(x)=-2\langle x, y\rangle$ Id for all $x, y \in \mathbf{R}^{n}$.

For example, $\operatorname{Spin}(3) \cong \operatorname{SU}(2)$ is the universal cover of $\operatorname{SO}(3)$, and $\rho$ is the standard representation of SU(2) on $\mathbf{C}^{2}$. In this case Clifford multiplication is simply

$$
c(x, y, z)=\left(\begin{array}{cc}
i x & i y+z \\
i y-z & -i x
\end{array}\right) \in \text { su(2). }
$$

For later use we note that the Lie algebra representation $\bar{\rho}$ : spin $(n) \rightarrow$ End $(V)$ can be written in terms of Clifford multiplication. Namely, for any skew-symmetric matrix $A \in$ so $(n) \cong \operatorname{spin}(n)$ we have

$$
\bar{\rho}(A)=-\frac{1}{4} A_{i j} c\left(e^{i}\right) c\left(e^{j}\right)=-\frac{1}{8} A_{i j}\left[c\left(e^{i}\right), c\left(e^{j}\right)\right],
$$

where $\left\{e^{i}\right\}$ is the standard basis of $\mathbf{R}^{n}$. (It is easy to verify that this is a Lie algebra representation.)

Now suppose $(N, g)$ is an oriented Riemannian manifold, and $F_{N}$ the bundle of oriented orthonormal frames for $T^{*} N$ (a principal $\operatorname{SO}(n)$ bundle). When the second Stiefel-Whitney class $\omega_{2}(N)$ vanishes we can choose a lift $\tilde{F}_{N}$ of $F_{N}$ to a principal $\operatorname{Spin}(n)$ bundle; in this case one says $N$ is a spin manifold. We then have an associated Hermitian vector bundle $S=\tilde{F}_{N} \times{ }_{\rho} V$ called the spin vector bundle, and a global Clifford multiplication $c: \Gamma\left(T^{*} N\right) \rightarrow$ $\Gamma(\operatorname{End}(S))$. A section $\psi \in \Gamma(S)$ is called a spinor on $N$, and for a 1 -form $\omega$ we will denote Clifford multiplication by $c(\omega) \psi=\omega \cdot \psi$.

The metric $g$ on $N$ gives a Levi-Civita connection on $F_{N}$, on $\tilde{F}_{N}$, and on all associated vector bundles. The composition of the covariant derivative $\nabla$ on $\Gamma(S)$ with Clifford multiplication gives a first-order elliptic operator

$$
\text { D: } \Gamma(S) \stackrel{\nabla}{\rightarrow} \Gamma\left(T^{*} N \otimes S\right) \stackrel{c}{\rightarrow} \Gamma(S)
$$

called the Dirac operator. If $\left\{e_{i}\right\}$ is an orthonormal frame on $U \subset N$, and $\left\{e^{i}\right\}$ the dual coframe, then on $U$

$$
\dot{D}=\sum e^{i} \cdot \nabla_{e_{i}}
$$


The square of this operator satisfies the important formula of Lichnerowicz:

$$
\boldsymbol{B}^{2}=\nabla^{*} \nabla+\frac{1}{4} S
$$

where $\nabla^{*} \nabla \psi=-\psi_{, i}{ }^{i}$ is the covariant Laplacian on spinors. It is this formula that Witten used in place of the Bochner formula (10.1).

To prove (A.2), choose $P \in M$ and an orthonormal frame $\left\{e_{i}\right\}$ with $\nabla e_{i}(P)=0$. Since $e^{i} \cdot e^{j} \cdot+e^{j} \cdot e^{i} \cdot=-2 \delta^{i j}$, at $P$ we have

$$
\begin{aligned}
\dot{\otimes}^{2} & =e^{i} \cdot e^{j} \cdot \nabla_{i} \nabla_{j}=\left(-\delta^{i j}+\frac{1}{2}\left[e^{i} \cdot, e^{j} \cdot\right]\right) \nabla_{i} \nabla_{j} \\
& =\nabla^{*} \nabla+\frac{1}{2} e^{i} \cdot e^{j} \cdot\left[\nabla_{i}, \nabla_{j}\right] .
\end{aligned}
$$

At $P$, the curvature endomorphism $R\left(e_{i}, e_{j}\right)=\left[\nabla_{i}, \nabla_{j}\right]$ acts on 1-forms via the skew-symmetric matrix $R\left(e_{i}, e_{j}\right)_{k l}=R_{k l i j}$, so by (A.1) its action on spinors is

$$
\bar{\rho}\left(R\left(e_{i}, e_{j}\right)\right) \psi=-\frac{1}{4} R_{k l i j} e^{k} \cdot e^{l} \cdot \psi .
$$

Thus

$$
B^{2}=\nabla^{*} \nabla-\frac{1}{8} R_{k l i j} e^{i} \cdot e^{j} \cdot e^{k} \cdot e^{l} \cdot .
$$

By the symmetries of the curvature tensor,

$$
\begin{aligned}
0 & =\left(R_{k l i j}+R_{k i j l}+R_{k j l i}\right) e^{i} \cdot e^{j} \cdot e^{k} \cdot e^{l} . \\
& =3 R_{k l i j} e^{i} \cdot e^{j} \cdot e^{k} \cdot e^{l} \cdot-6 R_{i j} e^{i} \cdot e^{j} . \\
& =3 R_{k l i j} e^{i} \cdot e^{j} \cdot e^{k} \cdot e^{l} \cdot+6 S .
\end{aligned}
$$

Then (A.2) follows.

Now suppose $N$ is asymptotically flat with metric $g_{i j}=\delta_{i j}+h_{i j}$, where $h_{i j} \in C_{-\tau}^{2, \alpha}(N), \tau>(n-2) / 2$. Orthonormalizing $\left\{d z^{i}\right\}$ yields an orthonormal coframe $e^{i}=d z^{i}+\frac{1}{2} h_{i k} d z^{k}+O\left(\rho^{-\tau-1}\right)$. The connection coefficients of this frame are

$$
\begin{aligned}
\bar{\Gamma}_{i j}^{k} & =-\left\langle\nabla_{i} e^{k}, e_{j}\right\rangle=\Gamma_{i j}^{k}-\frac{1}{2} \partial_{i} h_{j k}+O\left(\rho^{-2 \tau-1}\right) \\
& =\frac{1}{2}\left(\partial_{j} g_{i k}-\partial_{k} g_{i j}\right)+O\left(\rho^{-2 \tau-1}\right) .
\end{aligned}
$$

This orthonormal coframe induces a trivialization of $F_{N}$, and hence of the spin bundle $\tilde{F}_{N}$, over the asymptotic end $N_{\infty}$. With respect to this trivialization, the Dirac operator is (using (A.1) and the above formula)

$$
\not \psi=e^{i} \cdot \nabla_{i} \psi=e^{i} \cdot \partial_{i} \psi-\frac{1}{8}\left(\partial_{k} g_{i j}\right) e^{i} \cdot\left[e^{j} \cdot, e^{k} \cdot\right] \psi+O\left(\rho^{-2 \tau-1}\right) \psi .
$$

The trivialization also enables us to define 'constant spinors' on $N_{\infty}$. Choose such a constant spinor $\psi_{0}$ with $\left|\psi_{0}\right| \rightarrow 1$ at infinity, and extend it to a smooth spinor on $N$. It follows from (A.3) that $\dot{\theta}^{2} \psi_{0} \in C_{-\tau-2}^{0, \alpha}(N)$. Now note that $S \geqslant 0$ implies that

$$
\dot{\nabla}^{2}: C_{-\tau}^{2, \alpha}(N) \rightarrow C_{-\tau-2}^{0, \alpha}(N)
$$

is injective. Indeed, suppose $\dot{\nabla}^{2} \xi=0$ for some $\xi \in C_{-\tau}^{2, \alpha}(N)$. Then $\xi=O\left(\rho^{-\tau}\right)$ and $\nabla \xi=O\left(\rho^{-\tau-1}\right)$. Applying (A.2) to $\xi$ and integrating by parts we see that the boundary terms vanish, leaving

$$
0=\int_{N}\left\langle\xi, \dot{\nabla}^{2} \xi\right\rangle d V_{g}=\int_{N}|\nabla \xi|^{2}+\frac{1}{4} S|\xi|^{2} d V_{g} .
$$


Since $S \geqslant 0$ this shows that $\nabla \xi=0$, so $\nabla|\xi|^{2}=\langle\nabla \xi$, $\xi\rangle+\langle\xi, \nabla \xi\rangle=0$. Thus $|\xi|$ is a constant, which is zero since $\xi$ vanishes at infinity.

Theorem 9.2(d) also holds for the operator $\nabla^{*} \nabla$ acting on spinors (with essentially the same proof). Therefore (A.4) is an isomorphism, so there exists $\xi \in C_{-\tau}^{2, \alpha}(N)$ with $\dot{B}^{2} \xi=\dot{D}^{2} \psi_{0}$. The spinor $\psi=\psi_{0}-\xi$ then satisfies $\dot{\theta}^{2} \psi=0$. Furthermore, $\varphi=\dot{B} \psi=\varnothing \psi_{0}-\not \xi$ satisfies $\not \varphi=0$ and lies in $C_{-\tau-1}^{1, \alpha}(N)$, so integrating by parts as above shows that $\varphi \equiv 0$. We have thus obtained a spinor $\psi$ which is asymptotic to $\psi_{0}$ and satisfies $\varnothing \psi=0$.

Now apply (A.2) to $\psi$ and integrate by parts over the region $N_{R}=\{\rho \leqslant R\}$. This time there is a boundary term:

$$
\begin{aligned}
& \left.\int_{N_{R}}|\nabla \psi|^{2}+\frac{1}{4} S|\psi|^{2} d V_{g}=\operatorname{Re} \int_{S_{R}}\left\langle\psi, \nabla_{i} \psi\right\rangle e_{i}\right\lrcorner d V_{g} \\
& \left.=\operatorname{Re} \int_{S_{R}}\left(\left\langle\psi_{0}, \nabla_{i} \psi_{0}\right\rangle-\left\langle\psi_{0}, \nabla_{i} \xi\right\rangle-\left\langle\xi, \nabla_{i} \psi_{0}\right\rangle+\left\langle\xi, \nabla_{i} \xi\right\rangle\right) e_{i}\right\lrcorner d V_{g} .
\end{aligned}
$$

As in (A.3) we have

$$
\boldsymbol{\operatorname { R e }}\left\langle\psi_{0}, \nabla_{i} \psi_{0}\right\rangle=-\frac{1}{8} \operatorname{Re}\left(\partial_{k} g_{i j}\right)\left\langle\psi_{0},\left[e^{j} \cdot, e^{k} \cdot\right] \psi_{0}\right\rangle+O\left(\rho^{-2 \tau-1}\right)
$$

Since $\left[e^{j} \cdot, e^{k} \cdot\right]$ is skew-Hermitian, this is $O\left(\rho^{-2 \tau-1}\right)$ and so the first term in (A.5) vanishes as $R \rightarrow \infty$. Furthermore, since $\xi=O\left(\rho^{-\tau}\right), \nabla \xi=O\left(\rho^{-\tau-1}\right)$, and $\nabla \psi_{0}=O\left(\rho^{-\tau-1}\right)$, the third and fourth terms also vanish.

To analyze the remaining term, let $L_{i}$ denote the operator

$$
L_{i}=\nabla_{i}+e^{i} \cdot \dot{\theta}=\left(\delta_{i j}+e^{i} \cdot e^{j} \cdot\right) \nabla_{j}=\frac{1}{2}\left[e^{i} \cdot, e^{j} \cdot\right] \nabla_{j} .
$$

If $\alpha$ is the $(n-2)$-form $\left.\left.\alpha=\left\langle\left[e^{i} \cdot, e^{j} \cdot\right] \psi_{0}, \xi\right\rangle e_{i}\right\lrcorner e_{j}\right\lrcorner d V_{g}$, then

$$
\begin{aligned}
d \alpha & \left.=-2\left(\left\langle\left[e^{i} \cdot, e^{j} \cdot\right] \nabla_{j} \psi_{0}, \xi\right\rangle+\left\langle\left[e^{i} \cdot, e^{j} \cdot\right] \psi_{0}, \nabla_{j} \xi\right\rangle\right) e_{i}\right\lrcorner d V_{g} \\
& \left.=-4\left(\left\langle L_{i} \psi_{0}, \xi\right\rangle-\left\langle\psi_{0}, L_{i} \xi\right\rangle\right) e_{i}\right\lrcorner d V_{g} .
\end{aligned}
$$

Therefore, by Stokes' theorem and the fact that $\dot{\otimes} \xi=\dot{\otimes} \psi_{0}$, the second term in (A.5) is

$$
\begin{aligned}
& \left.\boldsymbol{\operatorname { R e }} \int_{S_{R}}\left\langle\psi_{0},\left(e^{i} \cdot \not D-L_{i}\right) \xi\right\rangle e_{i}\right\lrcorner d V_{g} \\
& \left.\quad=\operatorname{Re} \int_{S_{R}}\left(\left\langle\psi_{0}, e^{i} \cdot \not \psi_{0}\right\rangle-\left\langle L_{i} \psi_{0}, \xi\right\rangle\right) e_{i}\right\lrcorner d V_{g} .
\end{aligned}
$$

As before, $\left\langle L_{i} \psi_{0}, \xi\right\rangle=O\left(\rho^{-2 \tau-1}\right)$, while (A.3) gives

$$
\begin{aligned}
e^{i} \cdot \not B \psi_{0} & =-\frac{1}{8}\left(\partial_{k} g_{l j}+O\left(\rho^{-2 \tau-1}\right)\right) e^{i} \cdot e^{l} \cdot\left[e^{j} \cdot, e^{k} \cdot\right] \psi_{0} \\
& =-\frac{1}{4}\left(\partial_{k} g_{l j}+O\left(\rho^{-2 \tau-1}\right)\right) e^{i} \cdot e^{l} \cdot\left(\delta^{j k}+e^{j} \cdot e^{k} \cdot\right) \psi_{0} \\
& =-\frac{1}{4}\left(\partial_{j} g_{k j}-\partial_{k} g_{j j}+O\left(\rho^{-2 \tau-1}\right)\right) e^{i} \cdot e^{k} \cdot \psi_{0} .
\end{aligned}
$$

Writing $e^{i} \cdot e^{k} \cdot=\frac{1}{2}\left[e^{i} \cdot e^{k} \cdot\right]-\delta^{i k}$ and noting that $\left[e^{i} \cdot, e^{k} \cdot\right]$ is skew, we see that (A.6) becomes

$$
\left.\frac{1}{4} \int_{S_{R}}\left(\partial_{j} g_{i j}-\partial_{i} g_{j j}+O\left(\rho^{-2 \tau-1}\right)\right)\left|\psi_{0}\right|^{2} e_{i}\right\lrcorner d V_{g} .
$$


Putting this into (A.5) and letting $R \rightarrow \infty$ gives Witten's formula

$$
\int_{N}|\nabla \psi|^{2}+\frac{1}{4} S|\psi|^{2} d V_{g}=\frac{\omega}{4} m(g)
$$

The theorem follows immediately.

We remark that with a little more work the weighted Hölder spaces can be replaced by weighted Sobolev spaces in the above proof, and one need only assume that $g$ is asymptotically flat of order $\tau>(n-2) / 2$ and has finite mass.

\section{BIBLIOGRAPHY}

[ADM1] R. Arnowitt, S. Deser, and C. Misner, Canonical variables for general relativity, Phys. Rev. 117 (1960), 1595-1602.

[ADM2] _ Energy and the criteria for radiation in general relativity, Phys. Rev. 118 (1960), 1100-1104.

[ADM3] 122 (1961), $997-1006$.

[A1] T. Aubin, Problèmes isopérimétriques et espaces de Sobolev, J. Diff. Geom. 11 (1976), 533-598.

[A2] __ Équations différentielles non linéaires et problème de Yamabe concernant la courbure scalaire, J. Math. Pures Appl. 55 (1976), 269-296.

[A3] _ Nonlinear analysis on manifolds. Monge-Ampère equations, Springer-Verlag, New York, 1982.

[AM] P. Aviles and R. McOwen, Conformal deformation to constant negative scalar curvature on noncompact Riemannian manifolds, preprint.

[B] R. Bartnik, The mass of an asymptotically flat manifold, Comm. Pure Appl. Math. 34 (1986), 661-693.

[BL] H. Brezis and E. Lieb, A relation between pointwise convergence of functions and convergence of functionals, Proc. Amer. Math. Soc. 88 (1983), 486-490.

[BN] H. Brezis and L. Nirenberg, Positive solutions of nonlinear elliptic equations involving critical Sobolev exponents, Comm. Pure Appl. Math. 36 (1983), 437-477.

[C] M. Cantor, Elliptic operators and the decomposition of tensor fields, Bull. Amer. Math. Soc. (N.S.) 5 (1981), 235-262.

[CSCB] A. Chaljub-Simon and Y. Choquet-Bruhat, Problèmes elliptiques du second ordre sur une variété euclidienne à l'infini, Ann. Fac. Sci. Toulouse 1 (1978), 9-25.

[CBC] Y. Choquet-Bruhat and D. Christodoulou, Elliptic systems in $H_{s, \delta}$ spaces on manifolds which are Euclidean at infinity, Acta Math. 146 (1981), 129-150.

[E] L. Eisenhart, Riemannian geometry, Princeton University Press, Princeton, N. J., 1926.

[GHP] G. Gibbons, S. Hawking, and M. Perry, Path integrals and the indefiniteness of the gravitational action, Nucl. Phys. B138 (1978), 141-150.

[GT] D. Gilbarg and N. Trudinger, Elliptic partial differential equations of second order, Springer-Verlag, New York, 1983.

[G] R. Graham, A conformal normal form, in preparation.

[HE] S. Hawking and S. Ellis, The large scale structure of space-time, Cambridge University Press, Cambridge, 1973.

[JL1] D. Jerison and J. Lee, A subelliptic, nonlinear eigenvalue problem and scalar curvature on CR manifolds, Contemp. Math. 27 (1984), 57-63.

[JL2] _ The Yamabe problem on CR manifolds, J. Diff. Geom. 25 (1987) (to appear).

[JL3] Extremals for the Sobolev inequality on the Heisenberg group and the CR Yamabe problem, J. Amer. Math. Soc. 1 (1988) (to appear).

[JLA] _ Intrinsic CR normal coordinates and the CR Yamabe problem, in preparation.

[K] J. Kazdan, Some applications of partial differential equations to problems in geometry, Surveys in Geometry, Japan, 1983 (preprint). 
[KN] S. Kobayashi and N. Nomizu, Foundations of differential geometry. I, Interscience, New York, 1963.

[L] P.-L. Lions, Applications de la méthode de concentration-compacité à l'existence de fonctions extremales, C. R. Acad. Sci. Paris 296 (1983), 645-648.

[Lo] R. Lockhart, Fredholm properties of a class of elliptic operators on non-compact manifolds, Duke Math. J. 48 (1981), 289-312.

[M] R. McOwen, Behavior of the Laplacian on weighted Sobolev spaces, Comm. Pure Appl. Math. 32 (1979), 783-795.

[NW] L. Nirenberg and H. Walker, The null spaces of elliptic partial differential operators in $\mathbf{R}^{n}$, J. Math. Anal. Appl. 42 (1973), 271-301.

[O] M. Obata, The conjectures on conformal transformations of Riemannian manifolds, J. Diff. Geom. 6 (1971), 247-258.

[PR] T. Parker and S. Rosenberg, Invariants of conformal Laplacians, J. Diff. Geom., to appear.

[PT] T. Parker and C. Taubes, On Witten's proof of the positive energy theorem, Comm. Math. Phys. 84 (1982), 223-238.

[SU] J. Sacks and K. Uhlenbeck, The existence of minimal 2-spheres, Ann. of Math. 113 (1981), $1-24$.

[S] R. Schoen, Conformal deformation of a Riemannian metric to constant scalar curvature, J. Diff. Geom. 20 (1984), 479-495.

[SY1] R. Schoen and S.-T. Yau, On the proof of the positive mass conjecture in general relativity, Comm. Math. Phys. 65 (1979), 45-76.

[SY2] _ _ Proof of the positive action conjecture in quantum relativity, Phys. Rev. Lett. 42 (1979), 547-548.

[SY3] _ The energy and the linear momentum of space-times in general relativity, Comm. Math. Phys. 79 (1981), 47-51.

[SY4] _ Proof of the positive mass theorem. II, Comm. Math. Phys. 79 (1981), 231-260.

[SY5] The geometry and topology of manifolds of positive scalar curvature, in preparation. [SY6] __ Conformally flat manifolds, Kleinian groups and scalar curvature (preprint).

[Ta] G. Talenti, Best constant in Sobolev inequality, Ann. Mat. Pura Appl. 110 (1976), 353-372.

[T] N. Trudinger, Remarks concerning the conformal deformation of Riemannian structures on compact manifolds, Ann. Scuola Norm. Sup. Pisa 22 (1968), 265-274.

[We] S. Webster, Pseudohermitian structures on a real hypersurface, J. Diff. Geom. 13 (1978), 25-41.

[Wy] D. Weiyue, On a conformally invariant elliptic equation on $\mathbf{R}^{n}$, Comm. Math. Phys. 107 (1986), 331-335.

[W] E. Witten, $A$ new proof of the positive energy theorem, Comm. Math. Phys. 80 (1981), $381-402$.

[Y] H. Yamabe, On a deformation of Riemannian structures on compact manifolds, Osaka Math. J. 12 (1960), 21-37.

[Yau] S.-T. Yau, Problem section, Seminar on Differential Geometry (S.-T. Yau, ed.), Princeton University Press, Princeton, N. J., 1982.

Department of Mathematics, Harvard University, Cambridge, Massachusetts 02138

Department of Mathematics, Brandeis University, Waltham, MassachusetTs 02254 
